NASA Technical Memorandum 106749

\title{
$1 N-34$
}

ICOMP-94-6

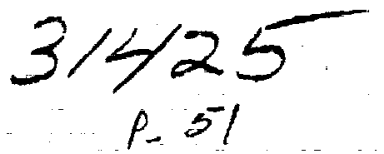

\section{On Bi-Grid Local Mode Analysis of Solution Techniques for 3-D Euler and Navier-Stokes Equations}

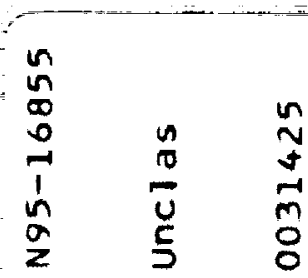

S.O. Ibraheem Old Dominion University

Norfolk, Virginia

and

A.O. Demuren Institute for Computational Mechanics in Propulsion Lewis Research Center Cleveland, Ohio and Old Dominion University Norfolk, Virginia

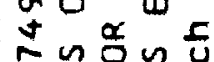
กษ出出

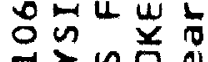
- $>$ n

October 1994

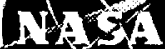

National Aeronautics and Space Administration 


\section{ABSTRACT}

A procedure is presented for utilizing a bi-grid stability analysis as a practical tool for predicting multigrid performance in a range of numerical methods for solving Euler and Navier-Stokes equations. Model problems based on the convection, diffusion and Burger's equation are used to illustrate the superiority of the bi-grid analysis as a predictive tool for multigrid performance in comparison to the smoothing factor derived from conventional von Neumann analysis. For the Euler equations, bi-grid analysis is presented for three upwind difference based factorizations, namely Spatial, Eigenvalue and Combination splits, and two central difference based factorizations, namely LU and ADI methods. In the former, both the Steger-Warming and van Leer flux-vector splitting methods are considered. For the Navier-Stokes equations, only the Beam-Warming (ADI) central difference scheme is considered. In each case, estimates of multigrid convergence rates from the bi-grid analysis are compared to smoothing factors obtained from single-grid stability analysis. Effects of grid aspect ratio and flow skewness are examined. Both predictions are compared with practical multigrid convergence rates for 2-D Euler and Navier-Stokes solutions based on the Beam-Warming central scheme. 


\section{Introduction}

Multiple grids were first proposed in the form of two-grid level schemes to accelerate the convergence of iterative procedures by researchers like Federenko [1]. Full multiple grid methods were later introduced by Federenko [2] to solve the Poisson equation and the approach was generalized by Bakhalov [3] to any second-order elliptic operator with continuous coefficients. According to Stuben and Trottenberg [4], Hackbush in [5] also independently developed some fundamental elements of the multigrid method. Perhaps the most influential work on the application of multigrid methods to elliptic type problems is that of Brandt [6] who also proposed the use of local mode analysis to determine the smoothing rate of multigrid schemes.

In local mode analysis, the spectral radius of a particular relaxation technique computed over only the high-frequency modes is used as a measure of the relaxation's effectiveness in a multigrid scheme since, in this case, the role of relaxation is not to reduce the total error but to smoothen it out i.e. remove the high-frequency components. It is assumed that the high-frequency modes have short wavelength that are spatially decoupled and that all high-frequency waves are completely 'killed' on the fine grid and are not visible to the coarse grids. This, however, is not always the case since the inter-grid processes also influence the convergence rate. Brandt [7] presented theoretical considerations for including the transfer processes in the local mode analysis in what is called the bi-grid method. Also, some theoretical background is given by Stuben and Trottenberg [4] on how to compute a more realistic amplification factor for multigrid methods based on the bi-grid analysis, where some convergence norms were computed for the Poisson and Helmholz equations.

A number of works exist where the smoothing factor has been used to predict multigrid performance in practice. However, the bi-grid analysis is becoming more attractive because of its better accuracy and reliability. van Asselt [8] used the bi-grid analysis to determine the proper amount of artificial viscosity to add at different level of coarse grids in a multigrid application. Mulder [9], [10] has also used the bi-grid method to construct an effective semi-coarsening in a multigrid method that can solve the problem of strong alignment which often occurs in convection problems. To select a relax- 
ation scheme for a multigrid method suitable for a parallel solution of a time-dependent problem, Horton and Vandewall [11] employed this technique using the heat equation as their model problem. The cause of the poor multigrid convergence rate that is experienced in high-Reynolds number flows, where the coarse grid corrections fail to approximate the fine grid problem well enough for certain components, has also been investigated by Brandt and Yavneh [12] using the bi-grid method. In an effort to develop an effective multigrid algorithm for Navier-Stokes solutions on an unstructured grid with $O(N)$ complexity, Morano [13], and Morano and Dervieux [14] have used the bigrid analysis on a 1-D model scalar convection equation with periodic boundary conditions. More recently, Ibraheem and Demuren [15] also presented some convergence norms for the Burger's equation based on bi-grid analysis.

Implicit numerical schemes are becoming very popular, since they allow large time steps for advancing the solution of Euler and Navier-Stokes equations to steady state. However, only few works exist to show the effectiveness of multigrid methods especially when approximate factorization is introduced. Yoon [16] and Caughey [17], for example, used the smoothing factor and scalar convection equation as a model for the Euler equations to investigate multigrid performance. Anderson et. al. [18], and Demuren and Ibraheem [19] have also computed the smoothing factors on the actual coupled Euler equations for some popular approximate factorizations. The latter work investigated the Navier-Stokes equations as well.

The objective of the present work is to present a procedure for utilizing the bi-grid amplification factor as a more practical tool for predicting multigrid performance in a range of numerical methods. $\mathrm{Bi}$-grid analysis, based on the von-Neumann type method, is first presented for 1-D convection and diffusion model problems, and the linearized Burger's equation. Numerical results from practical multigrid solution of these problems are compared to both predictions from bi-grid analysis and smoothing factors derived from the more usual single grid analysis. Both analyses and practical computations are based on the following different time-stepping methods: Euler forward explicit scheme, Runge-Kutta multistage scheme, a fully implicit scheme, and the semi-implicit scheme. The influence of the Peclet number on the convergence characteristics of the different schemes is 
investigated using the Burger's equation. Finally, for more practical situations, multigrid performance of various approximate factorizations for the 3-D Euler and Navier-Stokes equations are examined using the bi-grid stability analysis. For the Euler equations, bi-grid analysis is presented for three upwind difference-based factorizations and several central difference-based factorizations. In the upwind factorizations, both the flux-vector splitting methods of Steger-Warming and van Leer are considered. The central schemes include the Lower and Upper (LU) and ADI factorizations. The time-stepping algorithm for the Navier-Stokes equations is based on the Beam-Warming central difference scheme only. Practical multigrid solutions from numerical experiment on the ADI method are also compared to both predictions from bi-grid analysis and smoothing factors.

\section{Bi-grid Analysis}

Consider a given differential problem which can be written as:

$$
L\{u(x)\}=f(x) \quad \text {; for } x \text { in } \Omega
$$

where $\mathrm{L}$ is a linear operator. A typical 2-level multigrid cycle solution to this problem will involve the following steps:

(1) pre-relaxation on a fine grid using any technique $S_{1}, v^{1}$ times

(2) computation of the defect $R$

(3) restriction of the defect to the coarser grid

(4) exact solution of the error equation on the coarse grid

(5) prolongation of the error onto and the correction on the fine grid

(6) post-relaxation on the fine grid using any technique $S_{2}, v^{2}$ times

These can be represented for any intermediate solution $w$, by using usual operators as follows: 


$$
\begin{aligned}
& \text { (1) } w^{n+\frac{1}{2}}=S_{1}^{v^{1}} w^{n} \\
& \text { (2) } R=f-L_{h} w^{n+\frac{1}{2}} \\
& \text { (3) } I_{h}^{H} R \\
& \text { (4) } v_{H}=L_{H}^{-1}\left(I_{h}^{H} R\right) \\
& \text { (5) } I_{H}^{h} v_{H}+w^{n+\frac{1}{2}} \\
& \text { (6) } w^{n+1}=S_{2}^{v^{2}}\left(I_{H}^{h} v_{H}+w^{n+\frac{1}{2}}\right)
\end{aligned}
$$

Combining these steps, we can write:

$$
w^{n+1}=S_{2}^{\nu^{2}}\left[I_{H}^{h} L_{H}^{-1} I_{h}^{H}\left(f-L_{h} S_{1}^{\nu^{1}} w^{n}\right)+S_{1}^{\nu^{1}} w^{n}\right]
$$

The steady-state solution ( $u$ ) is not changed by the coarse grid correction scheme, thus

$$
u^{n+1}=S_{2}^{\nu^{2}}\left[I_{H}^{h} L_{H}^{-1} I_{h}^{H}\left(f-L_{h} S_{1}^{\nu^{1}} u^{n}\right)+S_{1}^{\nu^{1}} u^{n}\right]
$$

Subtracting (2) from (1) and noting that $e^{n+1}=u^{n+1}-w^{n+1}$ gives:

$$
\begin{aligned}
& \text { where } \quad \begin{aligned}
e^{n+1} & =S_{2}^{v^{2}}\left(\mathrm{I}-I_{H}^{h} L_{H}^{-1} I_{h}^{H} L_{h}\right) S_{1}^{\nu^{1}} e^{n} \\
& =S_{2}^{\nu^{2}} K S_{1}^{\nu^{1}} e^{n} \\
& =M e^{n} \\
K= & \mathrm{I}-I_{H}^{h} L_{H}^{-1} I_{h}^{H} L_{h} \\
M= & S_{2}^{\nu^{2}}\left(\mathrm{I}-I_{H}^{h} L_{H}^{-1} I_{h}^{H} L_{h}\right) S_{1}^{\nu^{1}}
\end{aligned}
\end{aligned}
$$

$M$ is the bi-grid amplification matrix and $K$ is the coarse grid correction matrix. It can be shown [4] that when linear operators are used for the restriction, $I_{h}^{H}$, and the prolongation, $I_{H}^{h}$ transfer processes, the coarse grid correction matrix is not a convergent iteration matrix. i.e.,

$$
\varrho(K)=\varrho\left(\mathrm{I}-I_{H}^{h} L_{H}^{-1} I_{h}^{H} L_{h}\right) \geq 1
$$

Hence, the fine grid smoothing steps $S_{1}$, and $S_{2}$ are important for a convergent scheme. The spectral radius of the bi-grid amplification matrix $\left(\lambda_{\max \_b g}\right)$ and its $l_{2}$ norm can be used to predict the performance of a multigrid method. While the spectral radius measures the asymptotic convergence rate of the multigrid method, the $l_{2}$ norm measures the actual error reduction per iteration. $\lambda_{\text {max } \_b g}$ is defined as follows: 


$$
\lambda_{\text {max } \_b g}=\max \{\varrho[\hat{M}(\Theta)]\}
$$

$\hat{M}(\Theta)$ is the Fourier representation of the matrix $M$. A brief comment about $\Theta$ will be in order. Due to aliasing process, low-frequency modes will couple with the coarse grid Fourier modes and, thus, for any $\Theta^{1}=\left\{\theta_{x}, \theta_{y}, \theta_{z}\right\}$ such that $-\pi / 2 \leq \theta_{x}, \theta_{y}, \theta_{z} \leq \pi / 2$ there exists a corresponding set of harmonics up to an integer multiple of $2 \pi$. For 1-D, 2-D and 3-D problems, we define $\Theta$ as the following set :

$$
\begin{aligned}
& \text { 1-D } \quad \Theta=\left\{\left(\theta_{x}\right),\left(\theta_{x} \pm \pi\right)\right\} \\
& \text { 2-D } \quad \Theta=\left\{\left(\theta_{x}, \theta_{y}\right),\left(\theta_{x}, \theta_{y} \pm \pi\right),\left(\theta_{x} \pm \pi, \theta_{y}\right),\left(\theta_{x} \pm \pi, \theta_{y} \pm \pi\right)\right\} \\
& \text { 3-D } \quad \Theta=\left\{\left(\theta_{x}, \theta_{y}, \theta_{z}\right),\left(\theta_{x}, \theta_{y}, \theta_{z} \pm \pi\right),\left(\theta_{x}, \theta_{y} \pm \pi, \theta_{z}\right),\left(\theta_{x}, \theta_{y} \pm \pi, \theta_{z} \pm \pi\right)\right. \text {, } \\
& \left(\theta_{x} \pm \pi, \theta_{y}, \theta_{z}\right),\left(\theta_{x} \pm \pi, \theta_{y}, \theta_{z} \pm \pi\right),\left(\theta_{x} \pm \pi, \theta_{y} \pm \pi, \theta_{z}\right), \\
& \left.\left(\theta_{x} \pm \pi, \theta_{y} \pm \pi, \theta_{z} \pm \pi\right)\right\}
\end{aligned}
$$

Or more generally,

$$
\text { d-D } \quad \Theta=\left\{\Theta^{1}, \Theta^{2}, \Theta^{3}, \ldots, \Theta^{2^{d}}\right\}
$$

(where $d$ is the dimensionality of the space, and $\Theta^{1}, \Theta^{2}, \ldots \Theta^{2^{d}}$ are permuted in a similar manner with the \pm signs chosen such that the harmonics lie in the high-frequency range).

Hence, based on the $\Theta$ components and on the number of degrees of freedom of the problem, $q$, $\hat{M}(\Theta)$ is a $2^{d} q \times 2^{d} q$ matrix. Thus, it is a $2 \times 2$ matrix for a $1-D$ scalar problem and $40 \times 40$ matrix for the Euler or Navier-Stokes equations in 3-D. The Fourier representation for the corresponding operators viz: smoothing factor, fine grid problem, interpolation, restriction and the coarse grid problem can be constructed as follows [7]:

$$
\begin{array}{ll}
\hat{S}=\left(\hat{S}_{2}^{v^{2}}, \hat{S}_{1}^{v^{1}}\right)=\operatorname{diag}\left[\hat{S}\left(\Theta^{1}\right), \hat{S}\left(\Theta^{2}\right), \ldots . \hat{S}\left(\Theta^{2^{d}}\right)\right] & 2^{d} q \times 2^{d} q \\
\hat{L}_{h}=\operatorname{diag}\left[\hat{L}\left(\Theta^{1}\right), \hat{L}\left(\Theta^{2}\right), \ldots . \hat{L}\left(\Theta^{2^{d}}\right)\right] & 2^{d} q X 2^{d} q \\
\hat{I}_{H}^{h}=\left[\hat{I}_{H}^{h}\left(\Theta^{1}\right), \hat{I}_{H}^{h}\left(\Theta^{2}\right), \ldots \ldots . \hat{I}_{H}^{h}\left(\Theta^{2^{d}}\right)\right] & 2^{d} q X q \\
\hat{I}_{h}^{H}=\left[\hat{I} \hat{I}_{h}^{H}\left(\Theta^{1}\right), \hat{I}_{h}^{H}\left(\Theta^{2}\right), \ldots . \hat{f}_{h}^{H}\left(\Theta^{2^{d}}\right)\right] & q X 2^{d} q \\
\hat{L}_{H}=\hat{L}\left(2 \Theta^{1}\right) & q X q
\end{array}
$$


The difference operator, $\hat{L}_{H}\left(2 \Theta^{1}\right)$, on the coarse grid is only qxq since the coarse grid problem is solved exactly.

$\hat{S}$ and $\hat{L}$ depend on the choice of the smoother and the governing equations, respectively. The transfer processes, however, are less problem-dependent. Following [7], the Fourier symbol of the prolongation operator based on a $I^{\text {th }}$-order polynomial is given by:

$$
\hat{I}_{H}^{h}\left(\Theta^{m}\right)_{k l}=\delta_{k l} \prod_{i=1}^{d} \psi_{I}\left(\cos \Theta_{i}^{m}\right) \quad m=1,2^{d}
$$

where $\psi_{2}(\xi)=(1+\xi) / 2, \psi_{4}(\xi)=\left(2+3 \xi-\xi^{2}\right) / 4$, etc. are the 2 nd and 4 th order interpolation functions, and $\delta_{k l}$ is the Kronecker delta. We restricted our analysis to the 2 nd order since it is more commonly used. The restriction operator is expressed as:

$$
2^{d} \hat{I}_{h}^{H}\left(\Theta^{m}\right)=\left[\hat{I}_{H}^{h}\left(\Theta^{m)}\right]^{T^{*}}\right.
$$

$T^{*}$ in the above equation represents the conjugate transpose. The restriction operator is often the adjoint of the prolongation operator in practice. In this study, the corresponding full weighting is used for the restriction operation for the Euler and Navier-Stokes equations, while simple injection is employed for the model problems. In the latter case, the Fourier symbol for the restriction operator is simply unity.

A description of how the Fourier representation $\hat{M}(\Theta)$ can be constructed is given later for certain problems.

\section{Model Equations}

The model equations used in the present study are the conservation equations for the convection of a scalar, the diffusion of a scalar, and the linearized Burger's equation which is essentially a convection-diffusion equation. Each of these equations is integrated in time using (i) Euler forward-explicit scheme, (ii) a Runge-Kutta multistage scheme, (iii) a fully implicit scheme and (iv) a semi-implicit scheme. 
The model equations for convection, diffusion, and the linear Burger's equation can be written as :

$$
\begin{array}{lll} 
& \text { convection : } & u_{t}+c u_{x}=0 \\
& \text { diffusion : } & u_{t}-v u_{x x}=0 \\
\text { (convection-diffusion) } & \text { Burger's : } & u_{t}^{*}+u_{o} u_{x}^{*}=v u_{x x}^{*}
\end{array}
$$

In the Burger's equation, $u_{o}=$ constant is assumed in our analysis. Thus, it can be put in the following non-dimensional form:

$$
u_{t}+u_{x}=\frac{1}{P e} u_{x x}
$$

where $P e$ in the above equation is the Peclet number defined as follows:

$$
P e=\frac{u_{0} D}{v}
$$

( $D$ is an appropriate length scale)

(i) Euler forward-explicit scheme

The Euler explicit method can be applied to the above equations to yield the following general discrete form:

$$
u_{i}^{n+1}=u_{i}^{n}-\Delta t R^{n}
$$

where $R^{n}$ represents the residual expressed as follows:

$$
\begin{array}{ll}
\text { convection: } & R^{n}=\frac{c}{\Delta x}\left(u_{i}^{n}-u_{i-1}^{n}\right) \\
\text { diffusion : } & R^{n}=-\frac{v}{\Delta x^{2}}\left(u_{i+1}^{n}-2 u_{i}^{n}+u_{i-1}^{n}\right) \\
\text { Burger's : } & R^{n}=\frac{1}{\Delta x}\left(u_{i}^{n}-u_{i-1}^{n}\right)-\frac{1}{\Delta x^{2} P e}\left(u_{i+1}^{n}-2 u_{i}^{n}+u_{i-1}^{n}\right)
\end{array}
$$

Space discretization in the above formulations is based on first-order upwind differences for convection, second-order central differences for diffusion, and the corresponding combination in the Burger's equation. First-order upwind differencing of the convective flux introduces inaccuracy due to too much numerical diffusion which may be of the same order of the natural diffusion in the Burger's equation. If second-order central differencing is used for the convective flux, a second-or- 
der accurate scheme can be obtained but with severe limitations on the Peclet number due to dispersion errors. Although the addition of artificial viscosity could dampen the high-frequency oscillations at high Peclet numbers, it is highly problem dependent. A better approach to achieve a second-order accuracy while sustaining a smooth solution at the vicinity of shock or high gradients is to discretize the convective flux using higher-order upwind schemes, preferably in conjunction with some limiter. Hence, with a third-order discretization of the convective flux, a second-order accurate scheme for the Burger's equation can be obtained with $R^{n}$ given by:

$$
\begin{aligned}
R^{n}= & \frac{1}{2 \Delta x}\left(u_{i+1}^{n}-u_{i-1}^{n}\right)-\frac{1}{6 \Delta x}\left(u_{i+1}^{n}-3 u_{i}^{n}+3 u_{i-1}^{n}-u_{i-2}^{n}\right) \\
& -\frac{1}{\Delta x^{2} P e}\left(u_{i+1}^{n}-2 u_{i}^{n}+u_{i-1}^{n}\right)
\end{aligned}
$$

\section{(ii) Runge-Kutta Multistage scheme}

With each of the above schemes integrated in time using the Euler forward explicit method, the time step was limited to a small range by stability considerations, thus making it inefficient for steadystate computations. A Runge-Kutta (RK) method was introduced by Jameson et. al. [18] to permit larger time steps to be taken. For an $\mathrm{m}$-stage scheme, the time integration can be written as follows:

$$
\begin{aligned}
& u_{i}^{o}=u_{i}^{n} \\
& u_{i}^{k}=u_{i}^{o}-a_{k} \Delta t R^{k-1} \quad k=1, m \\
& u_{i}^{n+1}=u_{i}^{m}
\end{aligned}
$$

Note that with $m=1$, the RK scheme reduces to the Euler forward explicit scheme and hence is sometime called RK1. Coefficients $\alpha_{k}$ are optimized such that larger time steps can be used for faster convergence.

Three different sets of coefficients for a 4-stage Runge-Kutta scheme are investigated in this study, in line with the earlier work of Morano [16]. These are the standard coefficients (RK4-S, $a_{1}=.25, a_{2}=.3333, \alpha_{3}=.5, \alpha_{4}=1$ ), and the optimized coefficients of Lallemand (RK4-L, $\left.a_{1}=.11, a_{2}=.2766, a_{3}=.5, a_{4}=1\right)$ and $\operatorname{van}$ Leer (RK4-VL, $a_{1}=.0833, a_{2}=.2069$, $a_{3}=.4265, a_{4}=1$ ). 


\section{(iii) Implicit scheme}

An implicit time integration scheme in delta form can easily be formulated for each of our model problems. For example, the corresponding implicit formulation for the Burger's equation with firstorder accuracy is written as follows:

$$
\begin{gathered}
{\left[-\beta\left(\frac{\Delta t}{\Delta x}+\frac{\Delta t}{P e \Delta x^{2}}\right)\right] \Delta u_{i-1}^{n}+\left[1+\beta\left(\frac{\Delta t}{\Delta x}+\frac{2 \Delta t}{P e \Delta x^{2}}\right)\right] \Delta u_{i}^{n}-\beta\left(\frac{\Delta t}{P e \Delta x^{2}}\right) \Delta u_{i+1}^{n}=-\Delta t R^{n}} \\
R^{n}=\frac{1}{\Delta x}\left(u_{i}^{n}-u_{i-1}^{n}\right)-\frac{1}{\Delta x^{2} P e}\left(u_{i+1}^{n}-2 u_{i}^{n}+u_{i-1}^{n}\right) \\
\Delta u_{i}^{n}=u_{i}^{n+1}-u_{i}^{n}
\end{gathered}
$$

$\beta$ in the above formulation is called the implicitness factor. $\beta=1.0$ gives a fully implicit scheme.

(iv) Semi-implicit scheme

If $\beta=0.5$ in equation (21) above we have a semi-implicit scheme. This reduces to the Crank-Nicolson scheme if the overall spatial differencing is second-order accurate.

\section{Fourier Symbols}

For illustration, the bi-grid amplification matrix $\hat{M}(\Theta)$ is constructed for the convection problem using the Euler-forward explicit scheme for the smoother:

Consider the discrete form of the operator $\mathrm{L}$ and let the step-by-step solution be characterized by Fourier modes (with periodic boundary conditions)

$$
u^{n}=U_{\partial} \lambda^{n} e^{\theta_{x} I i}
$$

Then each of the operators that forms matrix $\hat{M}(\Theta)$ becomes: 


$$
\begin{aligned}
& \hat{S}\left(\Theta^{m}\right)=\left(1-\frac{c \Delta t}{\Delta x}\right)+\frac{c \Delta t}{\Delta x}\left[\cos \left(\Theta^{m}\right)-I \sin \left(\Theta^{m}\right)\right] \\
& \hat{L}_{h}\left(\Theta^{m}\right)=-\frac{1}{\Delta x}\left[1-\cos \left(\Theta^{m}\right)+I \sin \left(\Theta^{m}\right)\right] \\
& \hat{I}_{H}^{h}\left(\Theta^{m}\right)=\frac{1}{2}\left[1+\cos \left(\Theta^{m}\right)\right] \quad m=1,2 \\
& \hat{I}_{h}^{H}\left(\Theta^{m}\right)=1 \quad \text { for injection } \\
& \hat{L}_{H}=-\frac{1}{2 \Delta x}\left[1-\cos \left(2 \Theta^{1}\right)+I \sin \left(2 \Theta^{1}\right)\right]
\end{aligned}
$$

where

$$
\Theta^{1}=\theta_{x} \text { and } \Theta^{2}=\theta_{x}+\pi
$$

Thus, from Eq. (8) $\hat{M}(\Theta)$ can be written as:

$$
\begin{aligned}
& \hat{M}(\Theta)=\left[\begin{array}{cc}
\hat{S}\left(\Theta^{1}\right) & 0 \\
0 & \hat{S}\left(\Theta^{2}\right)
\end{array}\right]^{\nu_{1}}\left[\begin{array}{ll}
K_{11} & K_{12} \\
K_{21} & K_{22}
\end{array}\right]\left[\begin{array}{cc}
\hat{S}\left(\Theta^{1}\right) & 0 \\
0 & \hat{S}\left(\Theta^{2}\right)
\end{array}\right]^{\nu_{2}}
\end{aligned}
$$

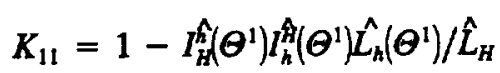

$$
\begin{aligned}
& \boldsymbol{K}_{12}=-\hat{I}_{H}^{h}\left(\Theta^{1}\right) \hat{I}_{h}^{\prime}\left(\Theta^{2}\right) \hat{L}_{k}\left(\Theta^{2}\right) / \hat{L_{H}} \\
& K_{21}=-\hat{I}_{H}^{h}\left(\Theta^{2}\right) \hat{I}_{h}^{A}\left(\Theta^{1}\right) \hat{L}_{h}\left(\Theta^{\prime}\right) / \hat{L}_{H} \\
& K_{22}=1-I_{H}^{\hat{H}}\left(\Theta^{2}\right) I_{h}^{\hat{H}}\left(\Theta^{2}\right) \hat{L_{h}}\left(\Theta^{2}\right) / \hat{L}_{H}
\end{aligned}
$$

Note that $\hat{L}_{H}$ is evaluated only at the fundamental frequency, hence it is $1 \times 1$. The result obtained above is similar to that derived by Morano [16], although our presentation is more general and is more easily extended to multi-dimensions.

\section{Multigrid Implementation}

A simple two-level multigrid ( $V$ cycle) method was implemented to test the relative accuracy of the bi-grid amplification factor and the smoothing factor in predicting multigrid performance. The two-level algorithm consists of the steps given in section 2 and is recursively expressible as follows: 


$$
\begin{aligned}
& \text { Proc Multigrid } \quad\left(u^{n}, u^{n+1}, R^{n}, k\right) \\
& \text { lif } \underset{\text { either }}{(k=1)} u^{n+1}=L_{H}^{-1} R^{n} \\
& \text { or } \quad u^{n+1}=S^{\infty} u^{n} \\
& \text { else } u^{n+1} \leftarrow S^{1} u^{n} \\
& R^{n} \leftarrow I_{h}^{H}\left(R^{n}-L u^{n}\right) \\
& \text { Multigrid }\left(0, u_{H}, R^{n}, k-1\right) \\
& u^{n+1} \leftarrow u^{n+1}+I_{H}^{h} u_{H} \\
& \text { endif \} }
\end{aligned}
$$

In the above, $\mathrm{L}$ and $\mathrm{S}$ stand for the discrete operator and relaxation scheme corresponding to each of the model equations and numerical schemes discussed in previous sections. For this two-level V cycle multigrid implementation, the exact solution of the residual equation is employed. Only one pre-relaxation with no post-relaxation is performed on the fine grid.

\section{Local Relaxation}

$\mathrm{Bi}$-grid analysis is exact for problems with periodic boundary conditions since it is based on the Fourier method. However, the asymptotic convergence rate for certain multigrid solutions deteriorates from the bi-grid prediction due to singularities such as discontinuity in material and /or solutions, and also due to the type and coefficients of the boundary conditions. Poor multigrid performance results since such singularities lead to too large a correction from the coarse grids in the localized region. To improve the performance of a multigrid solution, further relaxation can be performed on fine grid in the region of the singularities after applying the coarse grid correction. This local Relaxation is, infact, an extra post-relaxation but confined to only certain nodal points and carried out a few number of times. The extra computational work is negligible if only a few partial sweeps is involved. The convection dominated problems subject to Dirichlet boundary conditions that are considered here undergo high changes in gradient in order to satisfy the exit boundary conditions. Therefore, multigrid performance in these problems deviates from the results predicted by bigrid. However, a few passes on fine grid over the boundary conditions and over the interior equation in some small neighborhood of the boundary (about 3 nodal points at the exit) is found sufficient to improve multigrid performance to the exact value predicted by bi-grid analysis. 


\section{Numerical Experiments}

Bi-grid amplification factor $\left(\lambda_{\text {max } \_b g}\right)$, the smoothing factor $\left(\lambda_{\mu_{-} s g}\right)$ and the practical asymptotic convergence rate ( $\varrho_{m g}$ ) of the multigrid scheme were obtained for the following test problems:

(1) Convection problem with periodic boundary conditions, viz.:

$$
u(0, t)=u(1, t) \quad ; \quad u(x, 0)=\sin 2 \pi x
$$

(2) Convection problem with Dirichlet boundary conditions, viz:

$$
u(0, t)=1 \quad, \quad u(1, t)=0 \quad \text { for } t>0 \quad ; \quad u(x, 0)=\sin 2 \pi x
$$

(3) Diffusion problem with similar Dirichlet boundary conditions as in (2) above

(4) Burger's equation with similar Dirichlet boundary conditions as in (2) above.

The bi-grid amplification factor is obtained from Eq. (8) and the smoothing factor is obtained from the usual single grid amplification factor over the high frequency range $\pi / 2 \leq \Theta^{1} \leq \pi$ as $\lambda_{\mu_{-} s g}=\max \left\{\varrho\left[\hat{S}\left(\Theta^{1}\right)\right]\right\}$. In each case, sixteen Fourier modes are selected, and the associated eigenvalues are solved for using linear algebra routines such as found in the IMSL library. The asymptotic convergence rate of the multigrid experiments, on the other hand, is computed from [19]:

$$
\varrho_{m g}=\left(\frac{\left\|R^{n 2}\right\|}{\left\|R^{n 1}\right\|}\right)^{\frac{1}{n 2-n 1}}
$$

where $\left\|R^{n 1}\right\|$ and $\left\|R^{n 2}\right\|$ are the $l_{2}$ norm of the residuals at time levels $n 1$ and $n 2$, respectively.

The pseudotime $\Delta t$ to advance the convection and the diffusion problems to steady state is computed from $C F L=\frac{\Delta t}{\Delta x}$ and $d=\frac{\Delta t}{P e \Delta x^{2}}$, respectively. $C F L$ is the Courant-Friedrichs-Lewy number and $d$ is the diffusion number. For the Burger's equation, $\Delta t$ is computed from:

$$
\Delta t=\min \left(\sigma \Delta x, \sigma \Delta x^{2} P e\right)
$$


where $\sigma$ is an appropriate parameter chosen to reduce to the diffusion number $d$ at low $P e$ number and to the CFL number at high $P e$ number. This choice ensures that the appropriate time step is used in each flow regime. $\Delta x$ is computed from $\mathrm{D} / 20$. Preliminary tests showed that the same results are obtained with 40 or 80 points.

The exact steady-state solution for the Burger's equation, subject to the boundary condition type discussed above, is given by:

$$
u=u(0, t)\left[\frac{1-\exp \left[\operatorname{Pe}\left(\frac{x}{D}-1\right)\right]}{1-\exp (-P e)}\right]
$$

It is valid for all range of Pe considered in this study.

\section{Results for the Model Equations}

Figures 1 and 2 show results of the analyses of the 1-D convection equation using the Euler forward explicit scheme. The model problem of Fig. 1 has periodic boundary conditions whereas that of Fig. 2 has Dirichlet boundary conditions. The bi-grid analysis gives perfect prediction of practical multigrid performance in the former, whereas the smoothing factors from the single grid analysis are much too high. Both methods of analysis ignore boundary effects, so the same predictions are obtained in Figs. 1 and 2, and the analyses predictions are strictly correct only for problems with periodic boundary conditions. This is confirmed in Fig. 2(b) where the asymptotic multigrid convergence rate is now much worse than predicted by the bi-grid analysis. The reason for the degradation of the multigrid performance is the singularity which appears near the exit in Fig. 2(a). This degradation in performance could be cured with a few local relaxation sweeps [15], as shown in Fig. 2(c). Each sweep had marginal computational cost and 5 sweeps were sufficient to bring the multigrid performance for the Dirichlet problem in line with that with periodic boundary conditions and the prediction of the bi-grid analysis. Clearly the Euler forward explicit scheme does not have good convergence properties except for CFL numbers close to 0.5 , and it is divergent for CFL numbers greater than 1. Better convergence properties are achieved with Runge-Kutta (RK) schemes. Three 4-stage RK schemes were analyzed, and the results are shown in Fig. 3 for the 1-D convection prob- 
lem with periodic boundary conditions. With optimized coefficients Fig. 3(c), convergence could be obtained for CFL numbers up to 3. Further, bi-grid amplification factors below 0.4 are obtained for the range of $\mathrm{CFL}$ numbers from 0.5 to 2.5. There is also perfect agreement between the results of the bi-grid analysis and the practical multigrid convergence rates. Similar multigrid results were obtained by Morano [16]. Fig. 4 shows the result for the Dirichlet boundary conditions. In this case the multigrid convergence rates at higher CFL numbers are much better than predicted by either method. Clearly, the boundary effects are stronger with the RK scheme and there is no simple way to account for them in the analyses. Figure 5 shows results for a fully implicit scheme and for the semi-implicit Crank Nicolson scheme, for the 1-D convection equation. Although both schemes are stable for the whole range of $\mathrm{CFL}$ numbers, the $\mathrm{Crank}$ Nicolson scheme suffers from very poor convergence rate at high CFL numbers.

Results for the 1-D diffusion equation are presented in Figs. 6-8. Dirichlet boundary conditions are applied throughout, and the steady state solution is shown in Fig. 6(a). In each case, the bi-grid analysis gives perfect agreement with the multigrid convergence rate whereas the smoothing rate obtained from the single grid analysis is consistently too optimistic. On the whole, the predicted convergence rates for each method are similar to corresponding one obtained from the convection equation if the diffusion number, $d$ is replaced by the CFL number in the latter. Clearly, if the goal is to achieve rapid convergence to the steady state, the fully implicit scheme with high $d$ or CFL number is the obvious choice.

The linearized Burger's equation represents a mixed convection-diffusion problem. The whole range of model type from pure diffusion to pure convection can be obtained simply by varying the Peclet number from a very small value to a very large value. Computed results for 4 values of Pe $\left(10^{-4}, 20,100,10^{6}\right)$ are presented in Figs. 9-12, for the various discretization schemes considered here. The exact solution at the steady state is shown in Fig. 9(a), for the Dirichlet boundary conditions $\mathrm{u}(0, \mathrm{t})=1, \mathrm{u}(1, \mathrm{t})=0$. For high values of $P e$, there is a singularity near $\mathrm{x}=1$. As explained in section 6 local relaxation is performed to reduce the adverse effect of this singularity on the overall multigrid convergence rate. The results for the first-and second-order Euler time explicit schemes 
are presented in Figs. 9 and 10. In each case the bi-grid analysis gives quite good prediction of the multigrid convergence rate. On the other hand, single-grid analysis gives too optimistic estimates at low $\mathrm{Pe}$ and too pessimistic estimates at high $\mathrm{Pe}$. The second-order scheme shows much poorer convergence rates, especially at high $\mathrm{Pe}$. The results for the fully-implicit and semi-implicit schemes ate presented in Figs. 11 and 12. The superiority of the fully-implicit scheme is confirmed, especially for high Pe flows. For $\sigma$ (or CFL number) greater than 10, it is close to a direct solver with $\lambda \rightarrow 0$. In these cases too, the bi-grid analysis agrees quite well with the practical multigrid convergence rate, except near $\sigma=1$ in the semi-implicit scheme at high Pe. Because of the limited range of $\sigma$ where the convergence rate is much less that 1, the semi-implicit Crank Nicolson scheme is not a viable method for obtaining steady solutions for the model problem. If the main interest is rapid convergence to steady state, then the fully-implicit scheme at high values of $\sigma$ (or CFL number) will be optimum.

Presently bi-grid stability analysis has been presented for typical explicit and implicit solution methods for model problems which range from the diffusion equation to the convection equation and including the convection-diffusion equation at different Peclet numbers. For large scale practical computations, interest is really in solving the system of Euler or Navier-Stokes equations. In the following sections, the bi-grid stability analysis of fully-implicit schemes of Euler and NavierStokes equations are examined under various approximate factorization methods.

\section{Euler and Navier-Stokes Equations}

In order to extend the bi-grid analysis to the coupled equations of fluid flows, a discrete analog of these equations is formulated based on different approximate factorizations. The ADI factorization is formulated for the Navier-Stokes equations with the Euler equations as a degenerate case. Three different upwind factorizations and one central LU factorization formulated in [19] are, also, considered.

The 3-D Navier-Stokes equations in Cartesian coordinates can be written as 


$$
\frac{\partial Q}{\partial t}+\frac{\partial\left(E-E_{v}\right)}{\partial x}+\frac{\partial\left(F-F_{v}\right)}{\partial y}+\frac{\partial\left(G-G_{v}\right)}{\partial z}=0
$$

where $Q$ is the solution vector and $E, F, G$ are the conserved inviscid fluxes:

$$
\begin{aligned}
& Q=[\varrho, \varrho u, \varrho v, \varrho w, \varrho e]^{T} \\
& E=\left[\varrho u, \varrho u^{2}+p, \varrho u v, \varrho u w,(\varrho e+p) u\right]^{T} \\
& F=\left[\varrho v, \varrho v u, \varrho v^{2}+p, \varrho v w,(\varrho e+p) v\right]^{T} \\
& G=\left[\varrho w, \varrho w u, \varrho w v, \varrho w^{2}+p,(\varrho e+p) w\right]^{T}
\end{aligned}
$$

The viscous fluxes $E_{v}, F_{v}, G_{v}$ are:

$$
\begin{aligned}
E_{v}= & {\left[0, \frac{2}{3} \mu\left(2 u_{x}-v_{y}-w_{z}\right), \mu\left(u_{y}+v_{x}\right), \mu\left(u_{z}+w_{x}\right),\right.} \\
\left.\mu v\left(u_{y}+v_{x}\right)+\mu w\left(u_{z}+w_{x}\right)+\frac{2}{3} \mu u\left(2 u_{x}-v_{y}-w_{z}\right)+k T_{x}\right]^{T} & \\
F_{v}= & {\left[\begin{array}{c}
0, \mu\left(u_{y}+v_{x}\right), \frac{2}{3} \mu\left(2 v_{y}-u_{x}-w_{z}\right), \mu\left(v_{z}+w_{y}\right), \\
\left.\mu u\left(u_{y}+v_{x}\right)+\mu w\left(v_{z}+w_{y}\right)+\frac{2}{3} \mu v\left(2 v_{y}-u_{x}-w_{z}\right)+k T_{y}\right]^{T}
\end{array}\right.} \\
G_{v}= & {\left[\begin{array}{c}
0, \mu\left(w_{x}+u_{z}\right), \mu\left(v_{z}+w_{y}\right), \frac{2}{3} \mu\left(2 w_{z}-v_{y}-u_{x}\right), \\
\mu u\left(w_{x}+u_{z}\right)+\mu v\left(v_{z}+w_{y}\right)+\frac{2}{3} \mu w\left(2 w_{z}-v_{y}-u_{x}\right)+k T_{z}
\end{array}\right]^{T} }
\end{aligned}
$$

In above, $T=p /\left[\varrho c_{v}(\gamma-1)\right]$, and $p=(\gamma-1)\left[\varrho e-0.5\left(u^{2}+v^{2}+w^{2}\right)\right]$. Also, Stokes hypothesis $(\lambda=-(2 / 3) \mu)$ has been assumed. With $E_{v}, F_{\nu}, G_{\nu}$ set to zero, we recover the Euler equations.

Using the Beam-Warming scheme, the viscous fluxes are split directionally [20]. Following the approach presented in Anderson et al. [21] for 2-D Navier-Stokes equations, analysis yields the following ADI approximate factorization for the 3-D Navier-Stokes equations. Here, Euler time integration and constant fluid properties are assumed.

$$
\begin{aligned}
& {\left[\mathrm{I}+\Delta t\left(\delta_{x} A-\delta_{x x} R\right)\right]\left[\mathrm{I}+\Delta t\left(\delta_{y} B-\delta_{y y} S\right)\right]\left[\mathrm{I}+\Delta t\left(\delta_{z} C-\delta_{z z} Y\right)\right] \Delta Q=} \\
& -\Delta t\left[A \delta_{x}-R \delta_{x x}-R_{1} \delta_{y x}-R_{2} \delta_{z x}+B \delta_{y}-S_{1} \delta_{x y}-S \delta_{y y}-S_{2} \delta_{z y}+C \delta_{z}-Y_{1} \delta_{x z}-Y_{2} \delta_{y z}-Y \delta_{z z}\right] Q
\end{aligned}
$$


where the Jacobians $A, B, C$ are $\partial E / \partial Q, \partial F / \partial Q, \partial G / \partial Q$, respectively. The analytical expression for the viscous fluxes are given in Demuren and Ibraheem [19]. The right-hand side resulted from linearization and from assuming the flux Jacobians to be locally constant. To damp the high-frequency waves that will arise due to central differencing, second-order implicit $\left(D_{x}^{i}=-\varepsilon_{i} \Delta t \Delta x \delta_{x x}\right)$ and fourth-order explicit $\left(D_{x}^{e}=-\varepsilon_{e} \Delta t \Delta x^{3} \delta_{x x o x}\right)$ artificial dissipations are added as diagonal matrix coefficients in the numerical examples. Thus, with similar dissipations added in the $y$ and $z$ directions Eq. (34) becomes

$$
\begin{gathered}
{\left[\mathrm{I}+\Delta t\left(\delta_{x} A-\delta_{x x} R-\varepsilon \Delta x \delta_{x x}\right)\right]\left[\mathrm{I}+\Delta t\left(\delta_{y} B-\delta_{y y} S-\varepsilon, \Delta y \delta_{y y}\right)\right]\left[\mathrm{I}+\Delta t\left(\delta_{z} C-\delta_{z z} Y-\varepsilon, A z \delta_{z z}\right)\right]} \\
=-\Delta t\left[A \delta_{x}-R \delta_{x x}-R_{1} \delta_{y x}-R_{2} \delta_{z x}+B \delta_{y}-S_{1} \delta_{x y}-S \delta_{y y}-S_{2} \delta_{z y}\right. \\
\left.+C \delta_{z}-Y_{1} \delta_{x z}-Y_{2} \delta_{y z}-Y \delta_{z z}+\varepsilon_{e}\left(\Delta x^{3} \delta_{x x x}+\Delta y^{3} \delta_{y y y y}+\Delta z^{3} \delta_{z z z}\right)\right] Q
\end{gathered}
$$

The corresponding factorization for the Euler equations becomes apparent if the viscous flux Jacobians $R, R_{1}, R_{2}, S, S_{1}, S_{2}, Y, Y_{1}, Y_{2}$ are set to zero.

Other approximate factorizations that are considered in this work are those formulated for Euler equations in [18] and [19], viz:

$$
\begin{aligned}
& {\left[\mathrm{I}+\Delta t\left(\delta_{x}^{-} A^{+}+\delta_{x}^{+} A^{-}\right)\right]\left[\mathbf{I}+\Delta t\left(\delta_{y}^{-} B^{+}+\delta_{y}^{+} B^{-}\right)\right]\left[\mathbf{I}+\Delta t\left(\delta_{z}^{-} C^{+}+\delta_{z}^{+} C^{-}\right)\right] \Delta Q=-\Delta t R^{n}} \\
& {\left[\mathrm{I}+\Delta t\left(\delta_{x}^{-} A^{+}+\delta_{y}^{-} B^{+}+\delta_{z}^{-} C^{+}\right)\right]\left[\mathrm{I}+\Delta t\left(\delta_{x}^{+} A^{-}+\delta_{y}^{+} B^{-}+\delta_{z}^{+} C^{-}\right)\right] \Delta Q=-\Delta t R^{n}} \\
& {\left[\mathrm{I}+\Delta t\left(\delta_{x}^{-} A^{+}+\delta_{x}^{+} A^{-}+\delta_{z}^{-} C^{+}\right)\right]\left[\mathrm{I}+\Delta t\left(\delta_{y}^{-} B^{+}+\delta_{y}^{+} B^{-}+\delta_{z}^{+} C^{-}\right)\right] \Delta Q=-\Delta t R^{n}} \\
& \text { where } \quad R^{n}=\delta_{x}^{-} E^{+}+\delta_{x}^{+} E^{-}+\delta_{y}^{-} F^{+}+\delta_{y}^{+} F^{-}+\delta_{z}^{-} G^{+}+\delta_{z}^{+} G^{-} \\
& {\left[\mathrm{I}+\Delta t\left(\delta_{x}^{-} A_{1}+\delta_{y}^{-} B_{1}+\delta_{z}^{-} C_{1}\right)+x_{2} \Delta t\left(\delta_{x}^{-}+\delta_{y}^{-}+\delta_{z}^{-}\right)\right]} \\
& \mathrm{X}\left[\mathrm{I}+\Delta t\left(\delta_{x}^{+} A_{2}+\delta_{y}^{+} B_{2}+\delta_{z}^{+} C_{2}\right)-x_{2} \Delta t\left(\delta_{x}^{+}+\delta_{y}^{+}+\delta_{z}^{+}\right)\right] \\
& =-\Delta t\left(\delta_{x} E+\delta_{y} F+\delta_{z} G\right)-x_{4} \Delta t\left(\Delta x^{3} \delta_{x a x x}+\Delta y^{3} \delta_{y y y y}+\Delta z^{3} \delta_{z z z z}\right)
\end{aligned}
$$


Eqs. (36),(37) and (38) are upwind schemes, and are referred to as spatial, eigenvalue and combination factorizations, respectively. The flux-vector splitting methods of Steger-Warming [22] and van Leer [23] are assumed. Eq. (40) is the Lower and Upper (LU) factorization. Here, the fluxes devised by Jameson and Turkel [24], viz: $A_{1}=(A+|A|) / 2$ and $A_{2}=(A-|A|) / 2$, are used to achieve diagonal dominance. $\delta^{+}$and $\delta^{-}$denotes forward and backward difference operators, respectively, and $\varkappa_{2}$ and $x_{4}$ are the artificial dissipation coefficients.

\section{Fourier Symbols}

The bi-grid amplification matrix $\hat{M}(\Theta)$ is constructed from $M=S_{2}^{\nu^{2}}\left(\mathrm{I}-I_{H}^{h} L_{H}^{-1} I_{h}^{H} L_{h}\right) S_{1}^{\nu^{1}}$. For ease of presentation, the Euler equations alone are selected for illustration, with the ADI central scheme used as the smoother. In this case, viscous fluxes $R, R_{1}, R_{2}, S, S_{1}, S_{2}, Y, Y_{1}, Y_{2}$ are set to zero. The components operators of matrix $\hat{M}(\Theta)$ are:

(i) The fine/coarse grid Operator $\hat{L}$

The Euler equivalent form of Eq. (31) is:

$$
\frac{\partial Q}{\partial t}=-\left(\frac{\partial E}{\partial x}+\frac{\partial F}{\partial y}+\frac{\partial G}{\partial z}\right)+\text { dissipation }
$$

Thus, in quasi-linear form:

$$
\begin{aligned}
L(Q)=-\left(A \frac{\partial Q}{\partial x}+B \frac{\partial Q}{\partial y}+C \frac{\partial Q}{\partial z}\right) & +\varepsilon_{i}\left(\Delta x \frac{\partial^{2} Q}{\partial x^{2}}+\Delta y \frac{\partial^{2} Q}{\partial y^{2}}+\Delta z \frac{\partial^{2} Q}{\partial z^{2}}\right) \\
& -\varepsilon_{e}\left(\Delta x^{3} \frac{\partial^{4} Q}{\partial x^{4}}+\Delta y^{3} \frac{\partial^{4} Q}{\partial y^{4}}+\Delta z^{3} \frac{\partial^{4} Q}{\partial z^{4}}\right)
\end{aligned}
$$

Holding $A, B, C$ locally constant and employing second-order central differencing, the Fourier symbol of the fine grid problem on equal mesh size in all directions becomes:

$$
\begin{gathered}
\hat{L}_{h}\left(\Theta^{m}\right)=-\frac{I}{\Delta x}\left[A \sin \left(\Theta_{1}^{m}\right)+B \sin \left(\Theta_{2}^{m}\right)+C \sin \left(\Theta_{3}^{m}\right)\right]+\frac{2 \varepsilon_{i}}{\Delta x}\left[\cos \left(\Theta_{1}^{m}\right)+\cos \left(\Theta_{2}^{m}\right)+\cos \left(\Theta_{3}^{m}\right)-3\right] \\
-\frac{16 \varepsilon_{e}}{\Delta x}\left[\sin ^{4}\left(\frac{\Theta_{1}^{m}}{2}\right)+\sin ^{4}\left(\frac{\Theta_{2}^{m}}{2}\right)+\sin ^{4}\left(\frac{\Theta_{3}^{m}}{2}\right)\right] \mathrm{m}=1,8
\end{gathered}
$$


Note that $\Theta_{k}^{m}$ represent the $k^{\text {th }}$ element of the $\Theta^{m}$ component (see Eq. (9-11)).

For any arbitrary mode, Eq. (43) is a $40 \times 40$ matrix since each Jacobian is a $5 \times 5$ matrix and there are 8 harmonics including the fundamental mode. The coarse grid problem is assumed to be a version of the original problem on the fine grid and the coarse grid is formed simply by deleting every other fine grid point. Thus, the mesh size and Fourier modes are $\left\{2 \Delta x, 2 \Theta^{1}\right\}$ and its Fourier signature can be written as:

$$
\begin{aligned}
\hat{L}_{H}\left(2 \Theta^{1)}=-\frac{I}{2 \Delta x}\left[A \sin \left(2 \theta_{\mathfrak{r}}\right)\right.\right. & \left.+B \sin \left(2 \theta_{y}\right)+C \sin \left(2 \theta_{z}\right)\right]+\frac{\varepsilon_{i}}{\Delta x}\left[\cos \left(2 \theta_{x}\right)+\cos \left(2 \theta_{y}\right)\right. \\
+ & \left.\cos \left(2 \theta_{z}\right)-3\right]-\frac{8 \varepsilon_{e}}{\Delta x}\left(\sin ^{4} \theta_{x}+\sin ^{4} \theta_{y}+\sin ^{4} \theta_{z}\right)
\end{aligned}
$$

In the above equation, only the fundamental mode, $\Theta^{1}=\left\{\theta_{x}, \theta_{y}, \theta_{z}\right\}$, is employed since the coarse grid problem is assumed to be solved exactly. Hence, this is only a 5 X 5 matrix.

(ii) The relaxation Operator $\hat{S}$

Each of the equations (35), (36), (37), (38) and (40) can be expressed as

$$
N \Delta Q^{n}=-L=-\Delta t R^{n}
$$

von Neumann stability analysis is used on this system of linear equations by letting the step-by-step solution be characterized by

$$
Q^{n}=U_{d} \lambda^{n} e^{l i \theta_{x}} e^{l j \theta_{y}} e^{I k \theta_{z}}
$$

where $\lambda$ is the single grid amplification factor. Thus, Eq. (45) reduces to a complex generalized eigenvalue problem of the form

$$
\hat{K \mathbf{x}}=\lambda \hat{N} \mathbf{x} \text { where } \hat{K}=\hat{N}-\hat{L}
$$

The Fourier symbols of $\hat{N}$ and $\hat{L}$, for our particular example, can easily be shown to be 


$$
\begin{aligned}
& \hat{N\left(\Theta^{m}\right)}=\left[\mathrm{I}+\frac{\Delta t}{\Delta x}\left(A I \sin \left(\Theta_{1}^{m}\right)+4 \varepsilon_{i} \sin ^{2} \frac{\Theta_{1}^{m}}{2}\right)\right]\left[\mathrm{I}+\frac{\Delta t}{\Delta y}\left(B I \sin \left(\Theta_{2}^{m}\right)+4 \varepsilon_{i} \sin ^{2} \frac{\Theta_{2}^{m}}{2}\right)\right] \\
& \mathrm{X}\left[\mathrm{I}+\frac{\Delta t}{\Delta z}\left(C I \sin \left(\Theta_{3}^{m}\right)+4 \varepsilon_{i} \sin ^{2} \frac{\Theta_{3}^{m}}{2}\right)\right]
\end{aligned}
$$

$$
\hat{L}\left(\Theta^{m}\right)=\frac{\Delta t}{\Delta x} r\left(A \sin \left(\Theta_{1}^{m}\right)+B \sin \left(\Theta_{2}^{m}\right)+C \sin \left(\Theta_{3}^{m}\right)\right)+\frac{16 \Delta t \varepsilon_{e}}{\Delta x}\left(\sin ^{4} \frac{\Theta_{1}^{m}}{2}+\sin ^{4} \frac{\Theta_{2}^{m}}{2}+\sin ^{4} \frac{\Theta_{3}^{m}}{2}\right)
$$

The Fourier symbols corresponding to the other approximate factorizations are documented in Demuren and Ibraheem [25]. For each harmonic, $\Theta^{m}(m=1,8)$, Eq. (47) is solved to give five eigenvalues from which the elements of $\hat{S}(\Theta)$ are constructed. For example, if the eigenvalues corresponding to the mode $\Theta^{1}=\left\{\theta_{x}, \theta_{y}, \theta_{z}\right\}$ are $\Lambda=\left\{\lambda_{1}, \lambda_{2}, \lambda_{3}, \lambda_{4}, \lambda_{5}\right\}$, then, from Eq. (11), $\hat{S}\left(\Theta^{1}\right)=\Lambda \mathbf{L}$. The effective fine grid smoothing operation is obtained by raising the smoothing matrices to the power of $v^{1}$ and $v^{2}$, the pre- and post-smoothing counts, respectively.

\section{(iii) The Transfer Operators $\hat{I}_{H}^{h}$ and $I_{h}^{A}$}

For a second-order interpolation, the Fourier symbol of the prolongation operator, from Eq. (12), is:

$$
\hat{I}_{H}^{h}\left(\Theta^{m}\right)=\frac{1}{8}\left[1+\cos \left(\Theta_{1}^{m}\right)\right]\left[1+\cos \left(\Theta_{2}^{m}\right)\right]\left[1+\cos \left(\Theta_{3}^{m}\right)\right]
$$

The restriction operator, $\hat{I}_{h}^{H}$, is computed from this equation and Eq. (13) assuming full-weighing.

Based on the above operators, $\hat{M}(\Theta)$ is assembled from $M=S_{2}^{\nu^{2}}\left(I-I_{H}^{h} L_{H}^{-1} I_{h}^{H} L_{h}\right) S_{1}^{\nu^{1}}$. A symbolic form is given in Appendix A. It is an $8 \times 8$ block matrix of which each elemental block is a $5 \times 5$ matrix.

\section{Solution Procedure}

The eigenvalues for the bi-grid matrix $\hat{M}(\Theta)$ are computed from Eq. (8) over fixed Fourier modes to obtain the amplification factor. Sixteen modes are selected, in the range $-\pi / 2 \leq \Theta^{1} \leq \pi / 2$. The smoothing factor is also computed from the generalized eigenvalue problem (47) over only the high-frequency modes $\pi / 4 \leq\left|\Theta^{1}\right|<\pi / 2$ as $\lambda_{\mu_{-} s g}=\max (|\hat{l}|)$. In each case, the eigenvalues are 
solved for using the linear algebra routines such as found in the IMSL library. Uniform flow is assumed with $M_{\infty}=0.8$, zero yaw $\left(\alpha_{y}\right)$ and angle of attack $\left(\alpha_{a}\right)$, and $\gamma=1.4$. Further, the grid spacing is assumed to be uniform in all directions. Effects of aspect ratio and flow skewness are also investigated. The time-step and Reynolds number are calculated from

$$
\begin{aligned}
\Delta t & =\frac{C F L}{\left[\frac{|u|}{\Delta x}+\frac{|v|}{\Delta y}+\frac{|w|}{\Delta z}+c \sqrt{\frac{1}{\Delta x^{2}}+\frac{1}{\Delta y^{2}}+\frac{1}{\Delta z^{2}}}\right]} \\
\operatorname{Re} & =\frac{\ln \left(\sqrt{\Delta x^{2}+\Delta y^{2}+\Delta z^{2}}\right)}{\mu}
\end{aligned}
$$

Some other pertinent definitions used are as follows:

$$
\mathrm{I}=\sqrt{u^{2}+v^{2}+w^{2}}, \quad M_{\infty}=\frac{\mathrm{IM}}{a}, \quad v=u \tan \left(a_{y}\right), \quad w=u \tan \left(\alpha_{a}\right)
$$

Practical multigrid solutions are obtained for Euler and Viscous flows around a circular cylinder using the PROTEUS computer code developed at NASA Lewis Research Center. The FAS-FMG (full approximate storage-full Multigrid) algorithm applicable to non-linear systems of equations was implemented in the PROTEUS code [26]. Based on two levels, the asymptotic convergence rate of these flows were computed from Eq. (28). The Reynolds number based on the cylinder diameter is 20 and the Mach number is 0.2 . The grid size for the Euler flow is $25 \times 49$ and for the Viscous flow is $49 \times 49$. In each case, the grid was clustered such that the aspect ratio varied from 1.5 to 3.8 for the Euler flow and 0.5 to 12.2 for the Viscous flow. Further, the pre- and post-smoothing counts are 1 and 0 , respectively, as are assumed in the analyses too.

\section{Results for the Euler and Navier-Stokes Equations}

Figure 13 shows the convergence results for the 3-D Euler equations using the upwind schemes. The computed values for the smoothing factor $\left(\lambda_{\mu_{-} s g}\right)$ and bi-grid amplification factor $\left(\lambda_{\max \_} b g\right)$ for the spatial, eigenvalue and combination factorizations based on the Steger-Warming flux-vector splitting are shown in figures 13(a), 13(b) and 13(c), respectively. Both factors predict instability for the spatial split, especially for CFL number beyond 5.0. In the eigenvalue and combination factoriza- 
tions, better convergence characteristics are observed although the smoothing factor's prediction is slightly more optimistic. For these two factorizations, bi-grid analysis predicts near instability at CFL number above 25 whereas the smoothing factor predicts unconditional stability for all CFL numbers. Figures 13(d)-13(f) show predictions for multigrid performance of each factorization using the van Leer flux-vector splitting. Except for the spatial factorization, all the schemes are predicted unconditionally stable for all CFL numbers by both bi-grid and smoothing factors. The spatial factorization is stable only for CFL numbers below 12 and possesses better convergence characteristics at CFL number below 8 than the other two factorizations. From both analyses, i.e. from $\left(\lambda_{\mu_{-} s g}\right)$ and $\left(\lambda_{\max \_b g}\right)$ van Leer flux-vector splitting gives better convergence characteristics than the Steger-Warming method for multigrid procedures. It is observed that the present results of the smoothing factors for the van Leer method are similar to those presented by Anderson et. al.[18], and Demuren and Ibraheem [19].

Results for the 3-D Euler equations using the LU approximate factorization with central difference approximation and various levels of second-and fourth-order artificial viscosities, $\varkappa_{2}$ and $\varkappa_{4}$, are shown in Figs. 14(a)-14(c). Without the addition of second-order dissipations, i.e. $x_{2}=0$, the coefficients $\varkappa_{4}=0.3$ yields the optimal results (see fig. 14(a)). From figures 14(b) and 14(c), bigrid and smoothing factors predict that an appropriate combination of $\varkappa_{2}$ and $\varkappa_{4}$ (especially when $x_{4} \geq x_{2}$ ) can significantly improve the performance of the LU scheme when used as a relaxation scheme for multigrid. Also for all levels of dissipation, the smoothing factors estimates are more optimistic than the bi-grid results especially at lower CFL numbers.

The convergence characteristics for the 3-D Euler and Navier-Stokes equations for different levels of artificial dissipation and Reynolds numbers are shown in figures 14(d)-14(f) and 15, using the Beam-Warming (ADI) central difference scheme as the baseline solution algorithm. With no dissipation added to the Euler equations (fig. 14(d)), the bi-grid analysis predicts instability for all CFL numbers, while the smoothing factor predicts stability for $\mathrm{CFL}$ numbers below 15 . From figures 14(e) and 14(f), optimal multigrid performance is predicted by the bi-grid analysis for dissipation 
levels of $\varepsilon_{e}=0.5$ and $\varepsilon_{i}=1.0$. These results are similar to those obtained for the NavierStokes equations at $\operatorname{Re}=10^{6}$ (see figs. $15(\mathrm{~d})-15(\mathrm{f})$ ). With Reynolds number of 100 and no dissipation, both bi-grid and smoothing factors predict stability for certain range of CFL numbers although the latter is more optimistic. Also at this Reynolds number, the optimal dissipation levels are $\varepsilon_{e}=0.5$ and $\varepsilon_{i}=1.0$

All computations have been based on zero yaw and angle of attack, and also on uniform grid spacing in all directions. Sensitivities of convergence characteristics to flow skewness and aspect ratio are studied using the ADI central scheme at Reynolds number of 100 , and dissipation levels of $\varepsilon_{e}=0.5$ and $\varepsilon_{i}=1.0$. The results are shown in figures 16 and 17 . Generally, convergence characteristics are improved with increases in yaw angle at zero angle of attack although the range of stable CFL numbers becomes smaller (figs.16(a)-16(c)). From figures 16(d)-16(f), no significant difference is observed in the convergence results when the yaw and angle of attack are set equal to each other. From figure 17, the convergence characteristics become worse with increases in grid aspect ratio.

In order to ascertain the suitability of bi-grid and smoothing factors in predicting multigrid performance in complex flows, asymptotic convergence rate are computed from practical multigrid solutions of 2-D Euler and viscous flows around a circular cylinder. The steady-state solutions for these flows are shown in fig. 18(a). Rather than evaluating the corresponding bi-grid and smoothing factors from uniform flow conditions, as in previous cases, they are computed at each point in the flow field, thereby accounting for the variation in flow properties. Figs. 18(b) and 18(c) show estimates from both analyses based on the computed frozen coefficients of the Euler and viscous flows, respectively. These results are also summarized in Table I, and are compared with the asymptotic convergence rate measured from the practical multigrid computations. For both flow problems, smoothing factor deviates more from the practical solution than does the bi-grid factor. 


\section{Concluding Remarks}

Bi-grid stability analysis has been presented for typical explicit and implicit solution methods for model problems which range from the diffusion equation to the convection equation and including the convection-diffusion equation at different Peclet numbers. Bi-grid amplification factors were compared with smoothing factors and multigrid convergence rates. The predicted bi-grid amplification factors agree quite well with the asymptotic convergence rate of the multigrid method. The smoothing rate of the relaxation scheme obtained from a local mode analysis on a single grid is not an accurate predictor of the multigrid convergence rate. For multigrid performance in large scale practical computations, bi-grid amplification factor and smoothing factor were computed from the system of 3-D Euler and Navier-Stokes equations. Various approximate factorization methods that are popular in practice are considered. In typical practical multigrid solutions of 2-D Euler and viscous flow problems, bi-grid analysis is found to give a better prediction of the convergence rate than the smoothing factor obtained from a single grid analysis. 


\section{Acknowledgement}

This work is funded by NASA Lewis Research Center under Grant No. NAG-3-1329 with Dr. James Scott as Technical Monitor. Computations were performed on supercomputers at NASA Lewis and NASA Ames Research Centers. We are grateful to Dr. Eric Morano of ICASE, Langley Research Center for his advice and suggestions. 


\section{REFERENCES}

1. R. P. Federenko, A Relaxation Method for Solving Elliptic Difference Equations, Z Vycisl, Mat. Mat. Fiz. 1, 922 (1961).

2. R. P. Federenko, The speed of convergence of one iterative process, Z Vycisl, Mat. Mat. Fiz. 4, 559 (1964).

3. N. S. Bakhalov, On the convergence of a Relaxation Method with Natural Constraints on an Elliptic Operator, Z. Vycisl, Mat. Mat. Fiz. 6, 861 (1966).

4. K. Stuben and U. Trottenberg, Multigrid methods: Fundamental Algorithms, Model Problems Analysis and Applications, in Lecture notes in Mathematics, Multigrid Methods, 960 (SpringerVerlag, New York, 1982), p. 1.

5. W. Hackbusch, Ein iteratives verfahren zur schnellen Auflosung elliptisher Randevertprobleme, Report 76-12, Institut fur Angewandte Mathematika, Universitat Koln, 1976.

6. A. Brandt, Multi-level Adaptive Solutions to Boundary-value Problems, Math. Comp. 31, 138 (1977).

7. A. Brandt, Rigorous Quantitative Analysis of Multigrid, Preliminary Report Prepared for AirForce Office of Scientific Research, United States Air Force, 1991.

8. E. J. van Asselt, "The Multigrid Method and Artificial viscosity," in Lecture Notes in Mathematics, Multigrid Methods 960 (Springer-Verlag, NY, 1982 ), p. 313.

9. W. A. Mulder, "Analysis of a multigrid Method for the Euler Equations of Gas Dynamics in Two Dimensions," in Multigrid Methods, Theory, Applications and Supercomputing, Lecture Notes in Pure and Applied Mathematics, edited by S. F. McCormick, (Marcel Dekker Inc., New York, 1988), p. 467.

10. W. A. Mulder, A new Multigrid Approach to Convection Problems, J. of Comp. Phys., 83, 303 (1989). 
11. G. Horton and S. Vandewalle, A Space-Time Multigrid Method For Parabolic PDES, submitted to SIAM J. Sci. Computing (1993).

12. A. Brandt and I. Yavneh, Accelerated Multigrid Convergence and High-Reynolds Recirculating Flows, SIAM J. Sci. Computing, 14, 607 (1993).

13. E. Morano, Resolution des equation d'Euler par une methode multigrille stationaire, $P h D$ Thesis, (University de Nice Sophia-Antipolis, 1992), p. 50

14. E. Morano and A. Dervieux, Steady Relaxation Methods for Unstructured Multigrid Euler and Navier Stokes Equations, submitted to J. of Computational Fluid Dynamics (1993).

15. S. O. Ibraheem and A. O. Demuren, "Bi-grid Stability Analysis Method for Multigrid Prediction in Burger's Equation," in Proceedings, SIAM 18th Annual Meeting, Southeastern-Atlantic section, Winston-Salem, North Carolina, March 1994, p. 20.

16. A. Jameson and S. Yoon, Multigrid Solution of the Euler Equations Using Implicit Schemes, AIAA Paper 85-0293, 1985.

17. D. A. Caughey, Diagonal Implicit Multigrid Algorithm for the Euler Equations, AIAA J., 26, 841 (1988).

18. W. K. Anderson, J. L. Thomas and D. L. Whitfield, Three-Dimensional Multigrid Algorithmsfor the Flux-Split Euler Equations, NASA TP-2829, 1988.

19. A. O. Demuren and S. O. Ibraheem, On the Stability Analysis of Approximate Factorization Methods for 3D Euler and Navier-Stokes Equations, Numerical Heat Transfer, Part B, 25, 97 (1994), Also NASA TM-106314, 1993.

20. R. M. Beam and R. F. Warming, An Implicit Scheme for the Compressible Navier-Stokes Equations, AIAA J., 16, 393 (1978).

21. D. A. Anderson, J. C. Tannehill, and R. H. Pletcher, Computational Fluid Mechanics and Heat Transfer (McGraw-Hill, New York, 1984), p. 489. 
22. J. L. Steger and R. F. Warming, Flux Vector Splitting of the Inviscid Gasdynamic Equations with Application to Finite-Difference Methods, J. Comp. Phys. 40, 263 (1980).

23. B. van Leer, Flux-Vector Splitting for the Euler Equations, ICASE 82-30, 1982.

24. A. Jameson and E. Turkel, Implicit Schemes and LU Decompositions, Math. Comp. 37, 385 (1981).

25. A. O. Demuren and S. O. Ibraheem, Convergence Acceleration of the Proteus Computer Code with Multigrid Methods, Interim Report Prepared for the Internal Fluid Mechanics Division, NASA Lewis Research Center, 1992.

26. C. E. Towne, J. R. Schwab, T. J. Benson and A. Suresh, PROTEUS Two-Dimensional NavierStokes Computer Code - Version 1.0, Volumes 1-3, NASA TM's-102551-3, 1990. 
TABLE I: Convergence characteristics of 2-D Euler and Viscous flows around a cylinder

\begin{tabular}{|c|c|c|c|c|c|c|}
\hline \multirow{2}{*}{ CFL } & \multicolumn{4}{|c|}{ Euler } & \multicolumn{3}{c|}{ Viscous flow } \\
\cline { 2 - 7 } & $\lambda_{\mu_{-} s g}$ & $\lambda_{\max \_b g}$ & $\varrho_{m g}$ & $\lambda_{\mu_{-} s g}$ & $\lambda_{\max \_b}$ & $\varrho_{m g}$ \\
\hline 0.5 & 0.88 & 0.94 & 0.99 & 0.95 & 0.96 & 0.99 \\
\hline 1.0 & 0.80 & 0.92 & 0.98 & 0.91 & 0.94 & 0.98 \\
\hline 2.0 & 0.76 & 0.91 & 0.96 & 0.85 & 0.93 & 0.96 \\
\hline 4.0 & 0.81 & 0.90 & 0.93 & 0.77 & 0.92 & 0.94 \\
\hline 6.0 & 0.84 & 0.90 & 0.92 & 0.76 & 0.91 & 0.93 \\
\hline 8.0 & 0.87 & 0.90 & 0.92 & 0.81 & 0.91 & 0.92 \\
\hline 10.0 & 0.89 & 0.90 & 0.94 & 0.84 & 0.91 & 0.92 \\
\hline 12.0 & 0.91 & 0.90 & 0.95 & 0.87 & 0.91 & 0.92 \\
\hline
\end{tabular}




\section{APPENDIX A}

\section{The Bi-grid Amplification Matrix $\hat{M}(\Theta)$}

$$
\hat{M}(\Theta)=\left[\begin{array}{llllllll}
M_{11} & M_{12} & M_{13} & M_{14} & M_{15} & M_{16} & M_{17} & M_{18} \\
M_{21} & M_{22} & M_{23} & M_{24} & M_{25} & M_{26} & M_{27} & M_{28} \\
M_{31} & M_{32} & M_{33} & M_{34} & M_{35} & M_{36} & M_{37} & M_{38} \\
M_{41} & M_{42} & M_{43} & M_{44} & M_{45} & M_{46} & M_{47} & M_{48} \\
M_{51} & M_{52} & M_{53} & M_{54} & M_{55} & M_{56} & M_{57} & M_{58} \\
M_{61} & M_{62} & M_{63} & M_{64} & M_{65} & M_{66} & M_{67} & M_{68} \\
M_{71} & M_{72} & M_{73} & M_{74} & M_{75} & M_{76} & M_{77} & M_{78} \\
M_{81} & M_{82} & M_{83} & M_{84} & M_{85} & M_{86} & M_{87} & M_{88}
\end{array}\right]
$$

The diagonal elements are:

$$
\begin{aligned}
M_{11} & =\mathbf{I}-\hat{I}_{H}^{h}\left(\Theta^{1}\right) \hat{I}_{h}^{H}\left(\Theta^{1}\right) \hat{L}_{h}\left(\Theta^{1}\right) \hat{S}_{1}^{\nu_{1}}\left(\Theta^{1}\right) \hat{S}_{2}^{2} 2\left(\Theta^{1}\right) \hat{L}_{H}^{-1} \\
M_{22} & =\mathbf{I}-\hat{I}_{H}^{h}\left(\Theta^{2}\right) \hat{I}_{h}^{H}\left(\Theta^{2}\right) \hat{L}_{h}\left(\Theta^{2}\right) \hat{S}_{1}^{\nu_{1}}\left(\Theta^{2}\right) \hat{S}_{2}^{2}\left(\Theta^{2}\right) \hat{L}_{H}^{-1} \\
M_{33} & =\mathbf{I}-\hat{I}_{H}^{h}\left(\Theta^{3}\right) \hat{I}_{h}^{H}\left(\Theta^{3}\right) \hat{L}_{h}\left(\Theta^{3}\right) \hat{S}_{1}^{\nu}\left(\Theta^{3}\right) \hat{S}_{2}^{2}\left(\Theta^{3}\right) \hat{L}_{H}^{-1} \\
\vdots & \vdots \\
M_{88} & \doteq \mathbf{I}-\hat{I}_{H}^{h}\left(\Theta^{8}\right) \hat{I}_{h}^{H}\left(\Theta^{8}\right) \hat{L}_{h}\left(\Theta^{8}\right) \hat{S}_{1}^{\nu 1}\left(\Theta^{8}\right) \hat{S}_{2}^{\prime 2}\left(\Theta^{8}\right) \hat{L}_{H}^{-1}
\end{aligned}
$$

and the off-diagonal elements are:

$$
M_{n m}=-I_{h}^{\hat{n}}\left(\Theta^{n}\right) \hat{L}_{h}^{H}\left(\Theta^{m}\right) \hat{L}_{h}\left(\Theta^{m}\right) \hat{S}_{1}^{n_{1}}\left(\Theta^{n}\right) \hat{S}_{2}^{N_{2}}\left(\Theta^{\mathrm{m}}\right) L_{H}^{-1}
$$

where, for example,

$$
\begin{aligned}
& M_{21}=-\hat{I}_{H}^{h}\left(\Theta^{2}\right) \hat{I}_{h}^{H}\left(\Theta^{\prime}\right) \hat{L}_{k}\left(\Theta^{1}\right) \hat{S}_{1}^{1}\left(\Theta^{2}\right) \hat{S}_{2}^{2}\left(\Theta^{\prime}\right) \hat{L}_{H}^{-1} \\
& M_{32}=-\hat{I}_{H}^{h}\left(\Theta^{3}\right) \hat{I}_{h}^{H}\left(\Theta^{2}\right) \hat{L}_{h}\left(\Theta^{2}\right) \hat{S}_{1}^{1}\left(\Theta^{3}\right) \hat{S}_{2}^{\hat{A}_{2}}\left(\Theta^{2}\right) \hat{L}_{H}^{-1} \\
& M_{84}=-\hat{I}_{H}^{h}\left(\Theta^{8}\right) \hat{L}_{h}^{H}\left(\Theta^{4}\right) \hat{L}_{h}\left(\Theta^{4}\right) \hat{S}_{1}^{1}\left(\Theta^{8}\right) \hat{S}_{2}^{2}\left(\Theta^{4}\right) \hat{L}_{H}^{-1} \\
& M_{76}=-\hat{I}_{H}^{h}\left(\Theta^{7}\right) \hat{L}_{h}^{H}\left(\Theta^{6}\right) \hat{L}_{h}\left(\Theta^{6}\right) \hat{S}_{1}^{A_{1}}\left(\Theta^{7}\right) \hat{S}_{2}^{\hat{A}_{2}}\left(\Theta^{6}\right) \hat{L}_{H}^{-1}
\end{aligned}
$$

Each element is a $5 \times 5$ matrix corresponding to the 5 dependent variables in equation (31). 
(a)

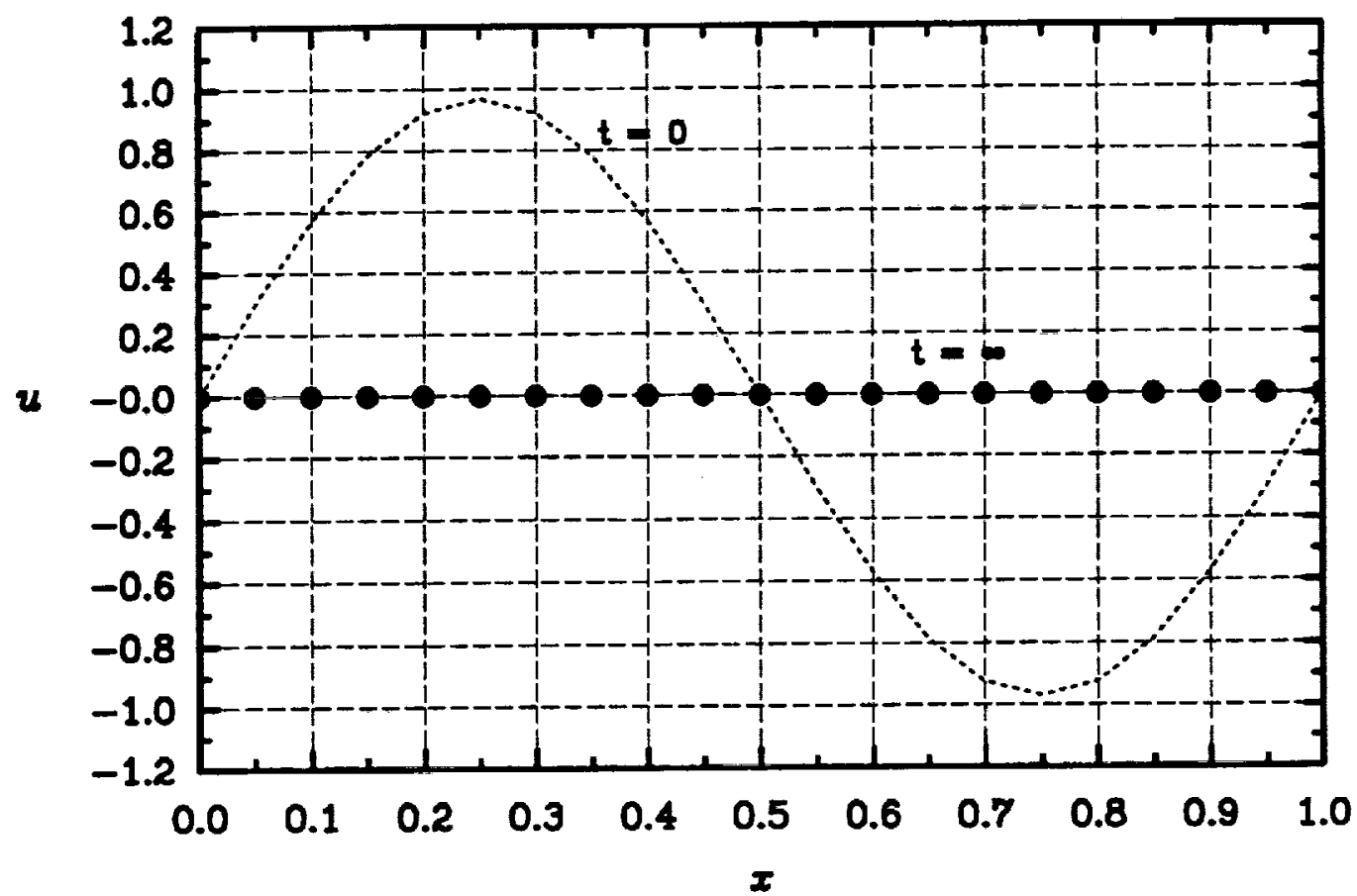

(b)

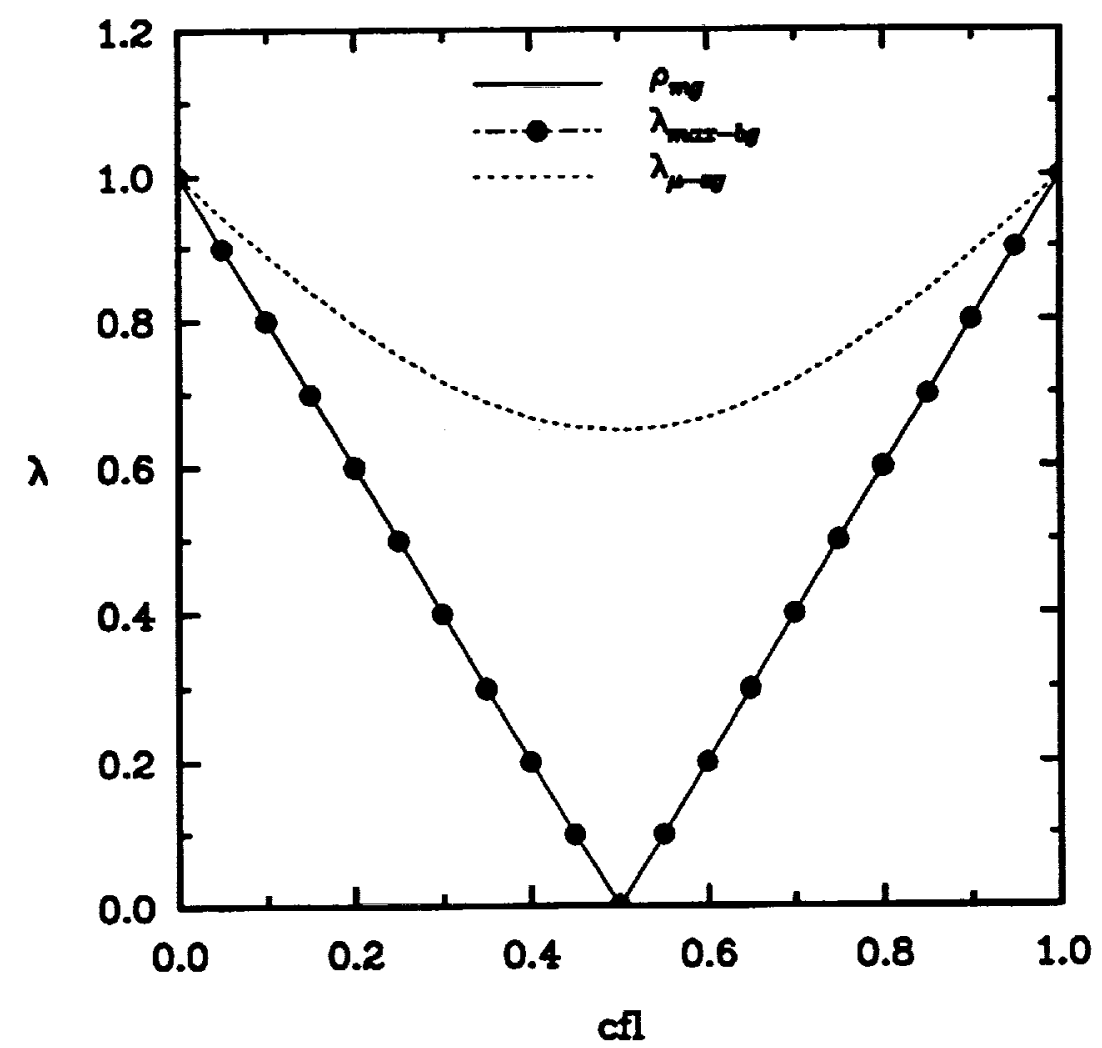

Fig. 1 1-D Convection Equation (a) Steady solution (b) Convergence Characteristics (Eular forward explicit; Periodic B.C's) 
(a)

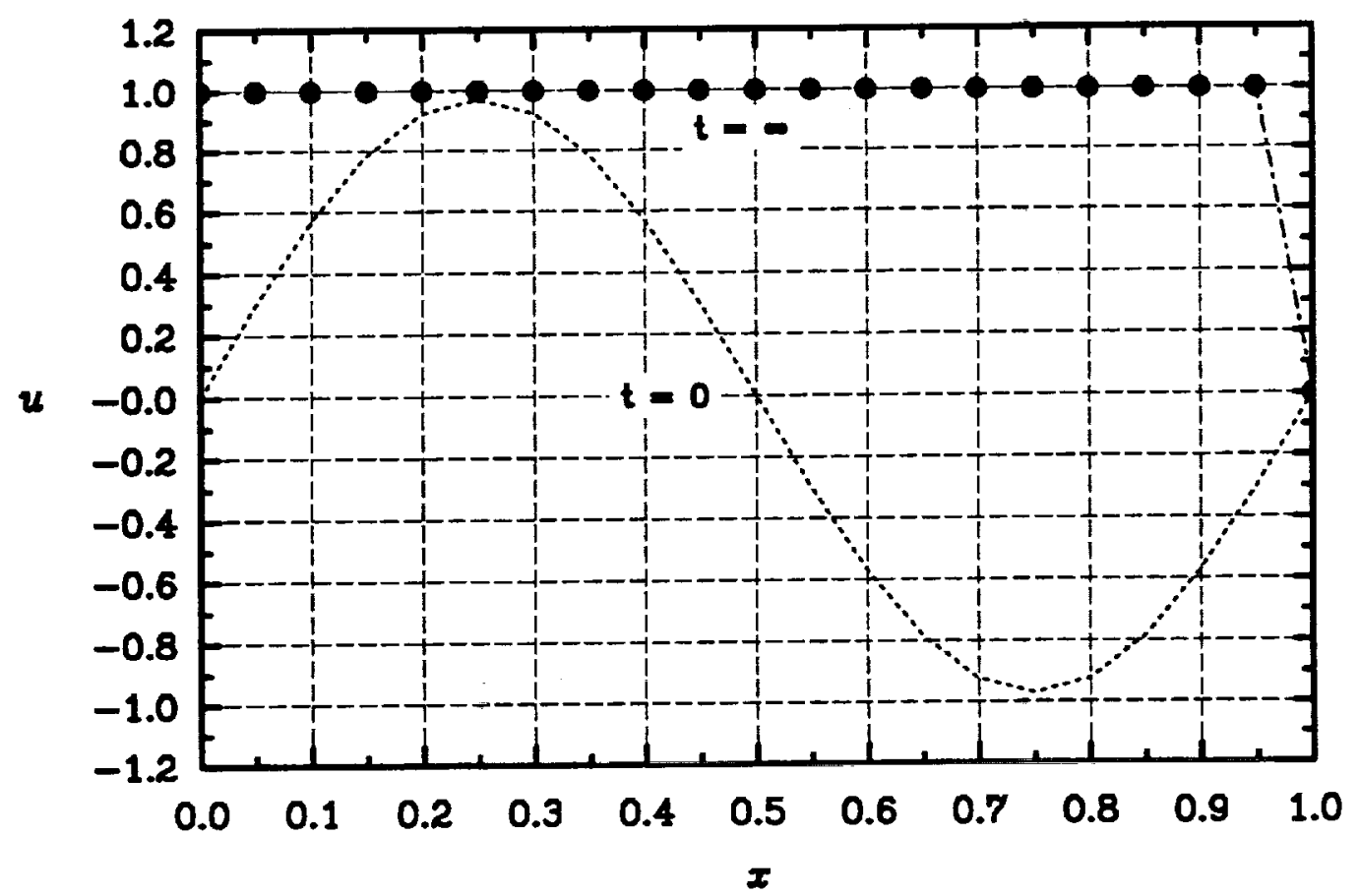

(b)

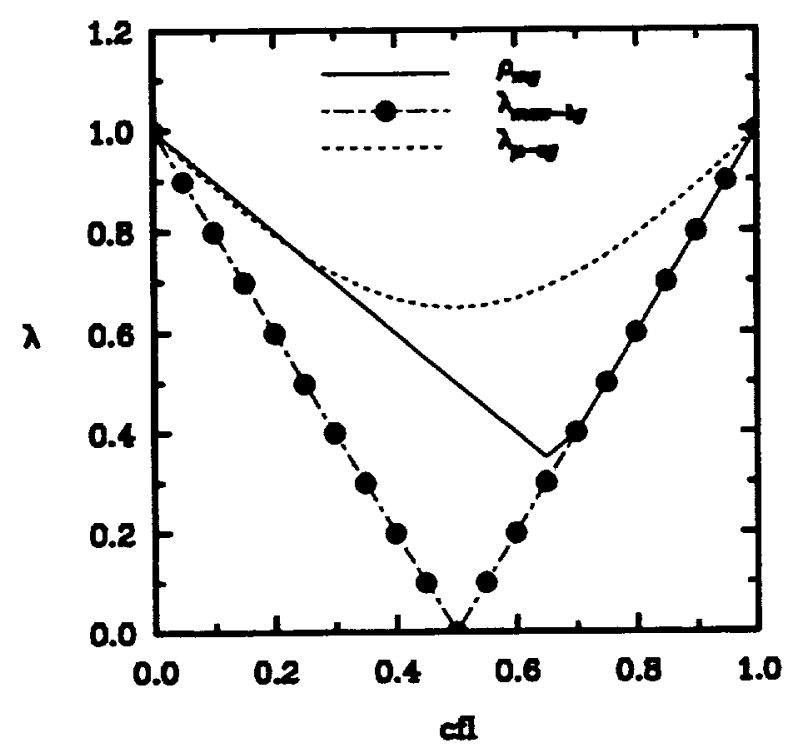

(c)

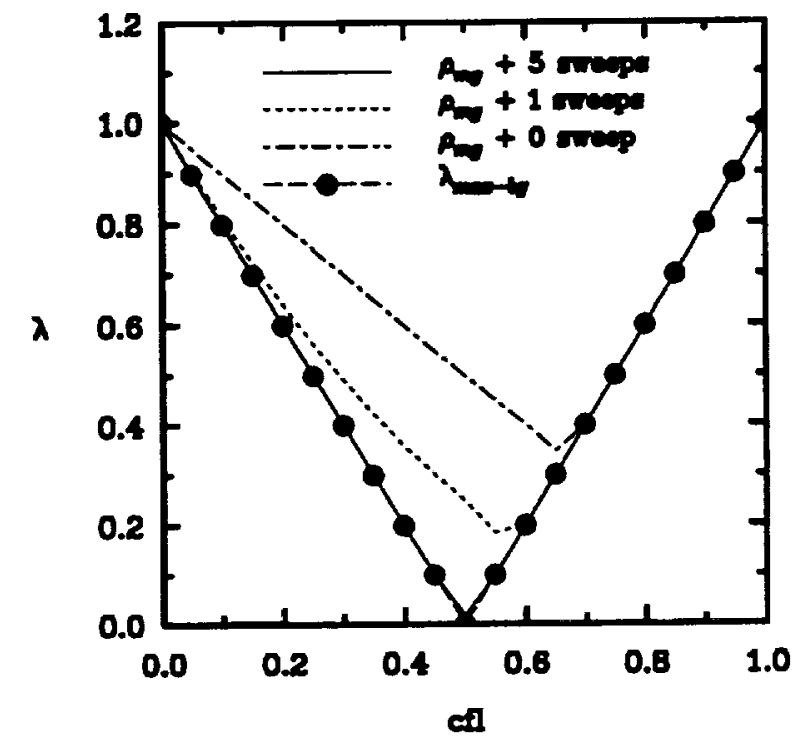

Fig. 2: 1-D Convection Equation (a) Steady solution (b) Convergence Charactaristica without local relaration (c) Convergence Charactoristics with local relaration (Buler forward explicit; Dirichlet B.C"s) 

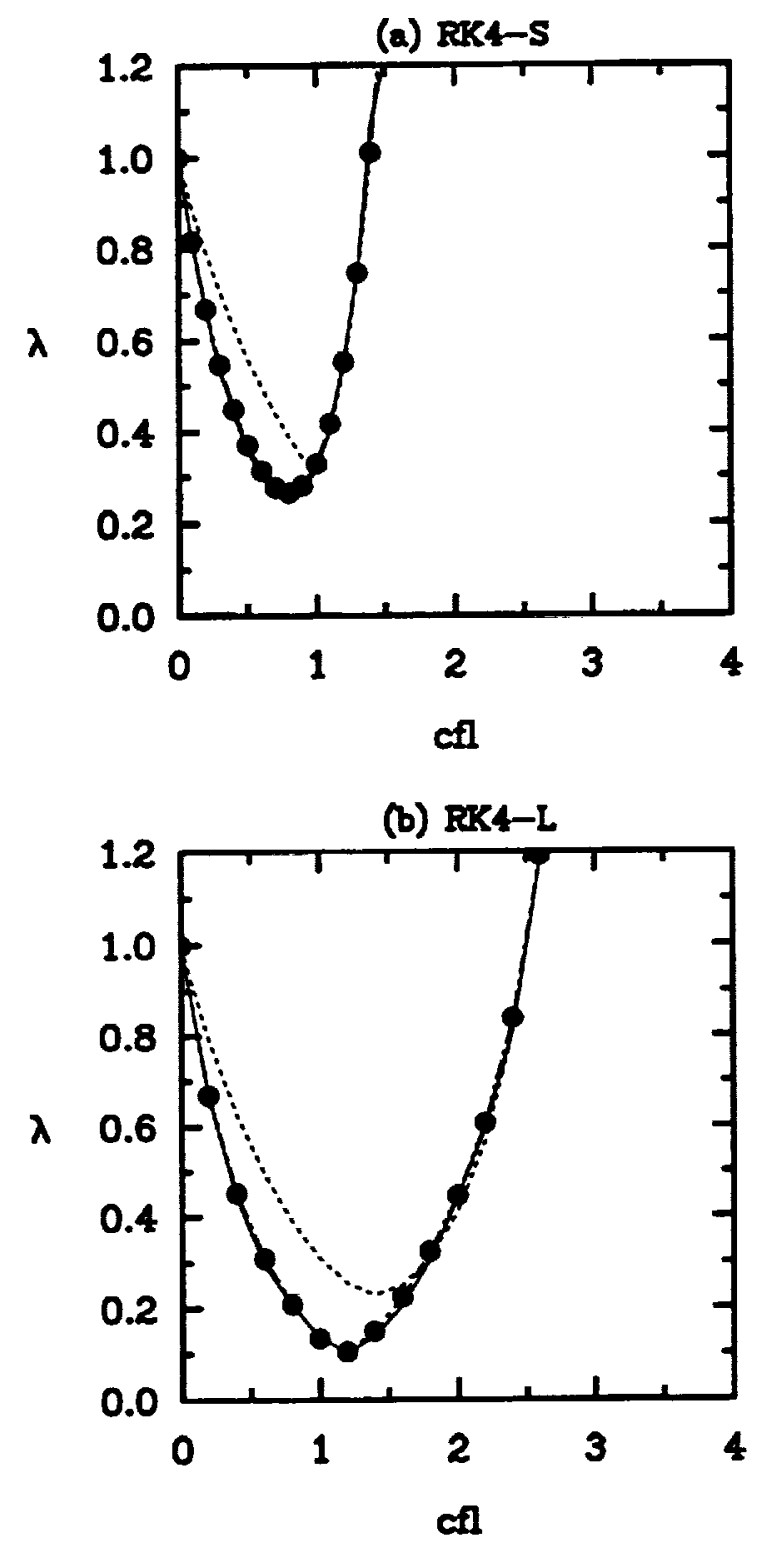

(c) $R \times 4 \leq-V 2$

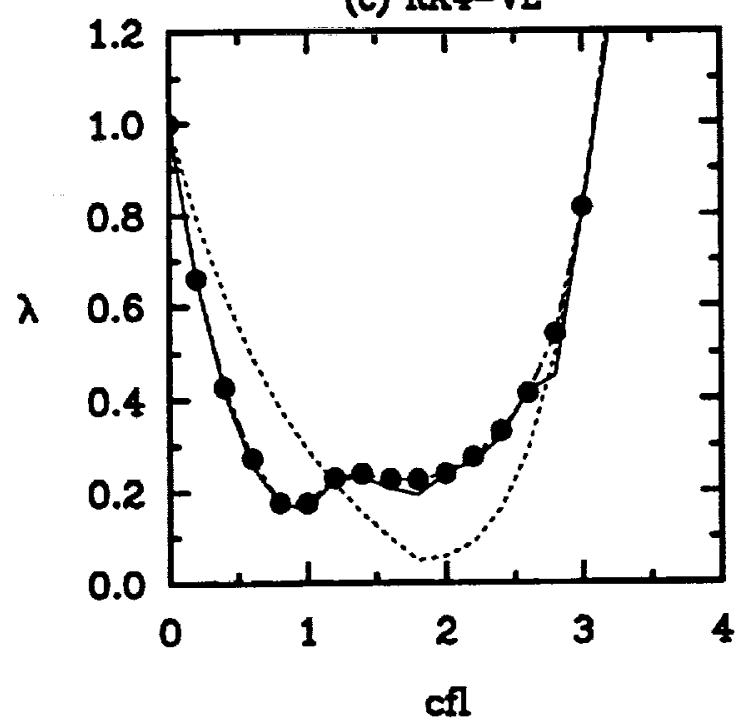

F8. 3 :Convergence Characteristics for 1-D Convection Equation

(a) Standard (b) Lollamad (c) van Loor coefflcients

(4-Stage Runge Kutta; Periodic B.C's) 


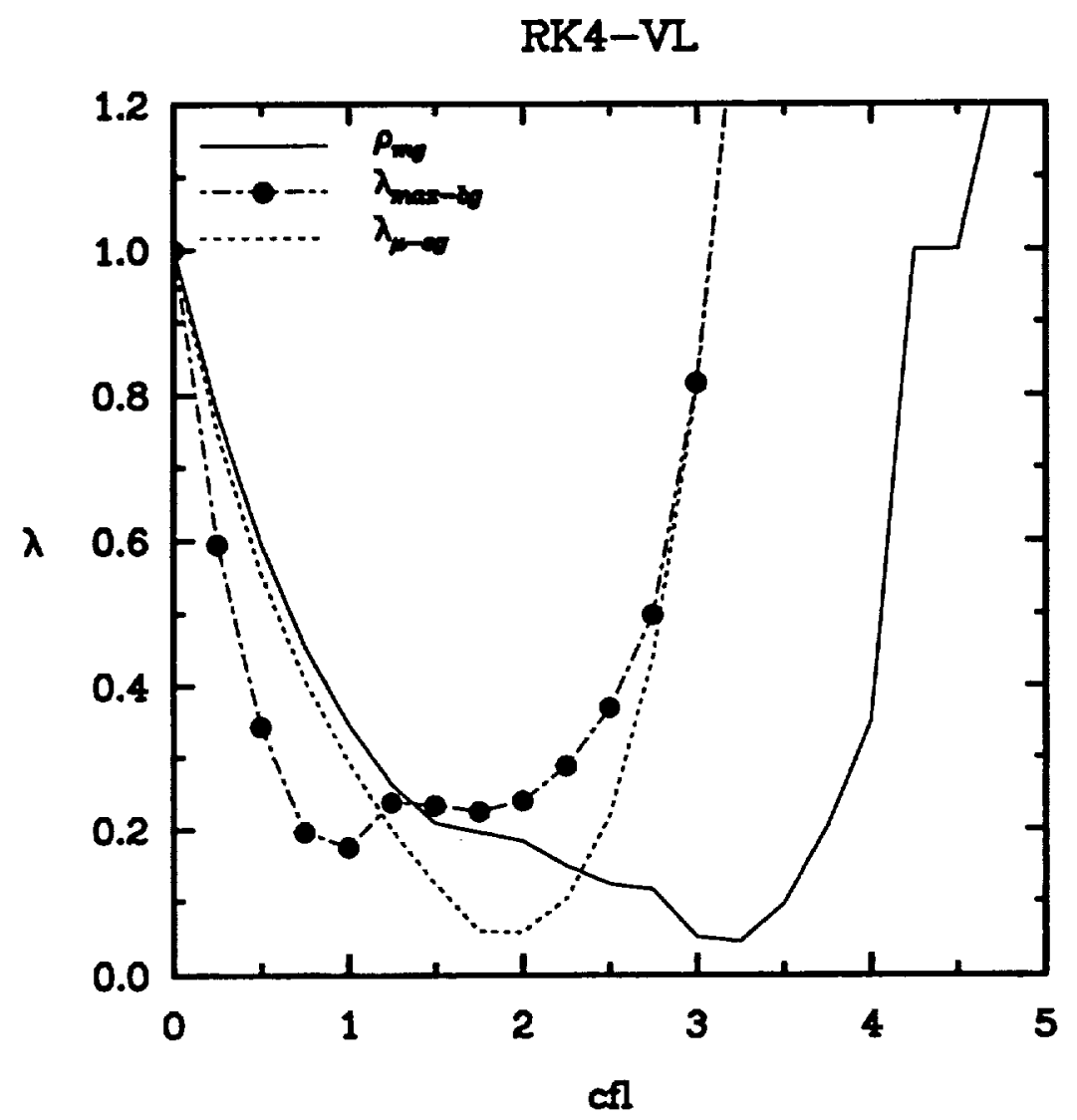

Fig. 4: Convergence Characteristics for 1-D Convection Equation (4-Stage Runge Kutta; Dirichlet B.C's; van Loor coefficients) 
(a)

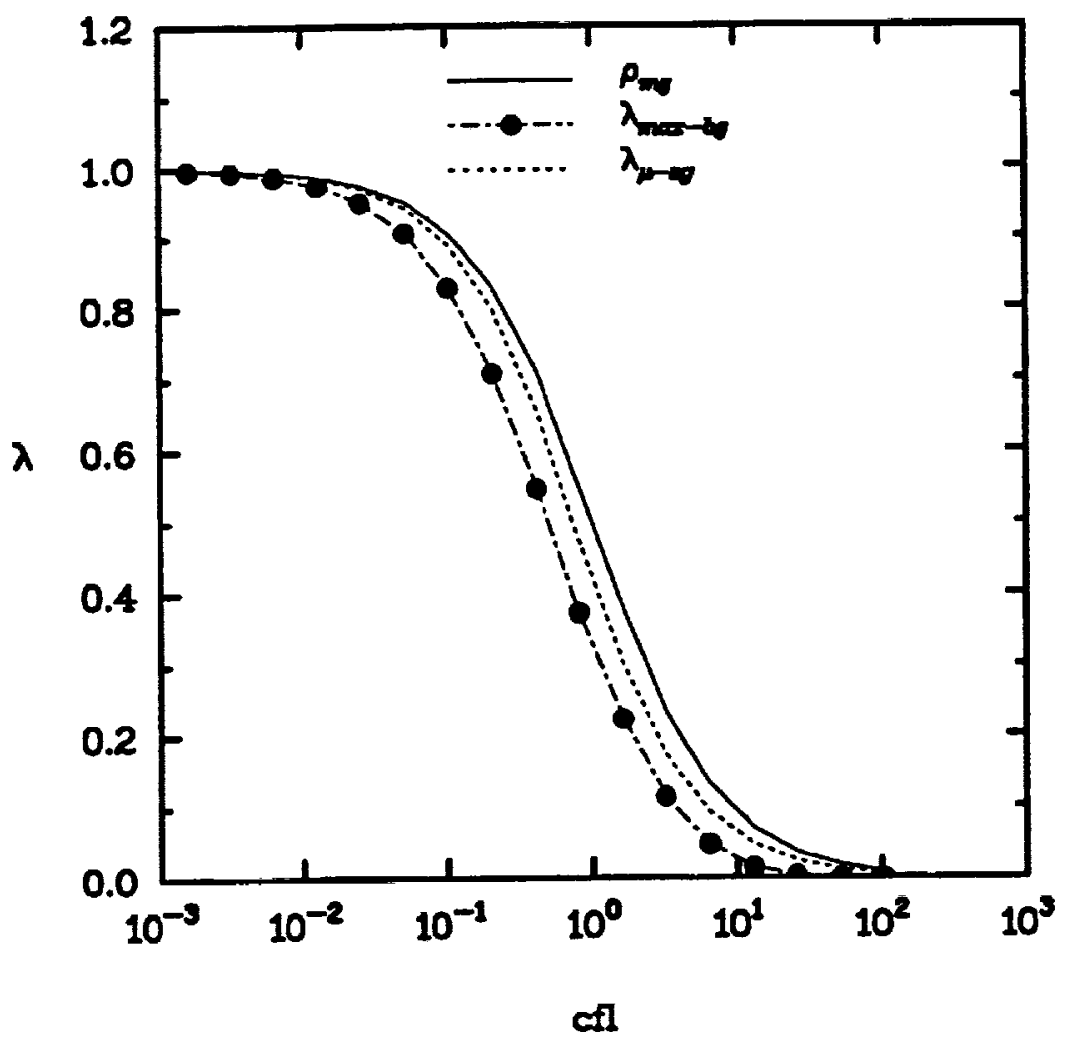

(b)

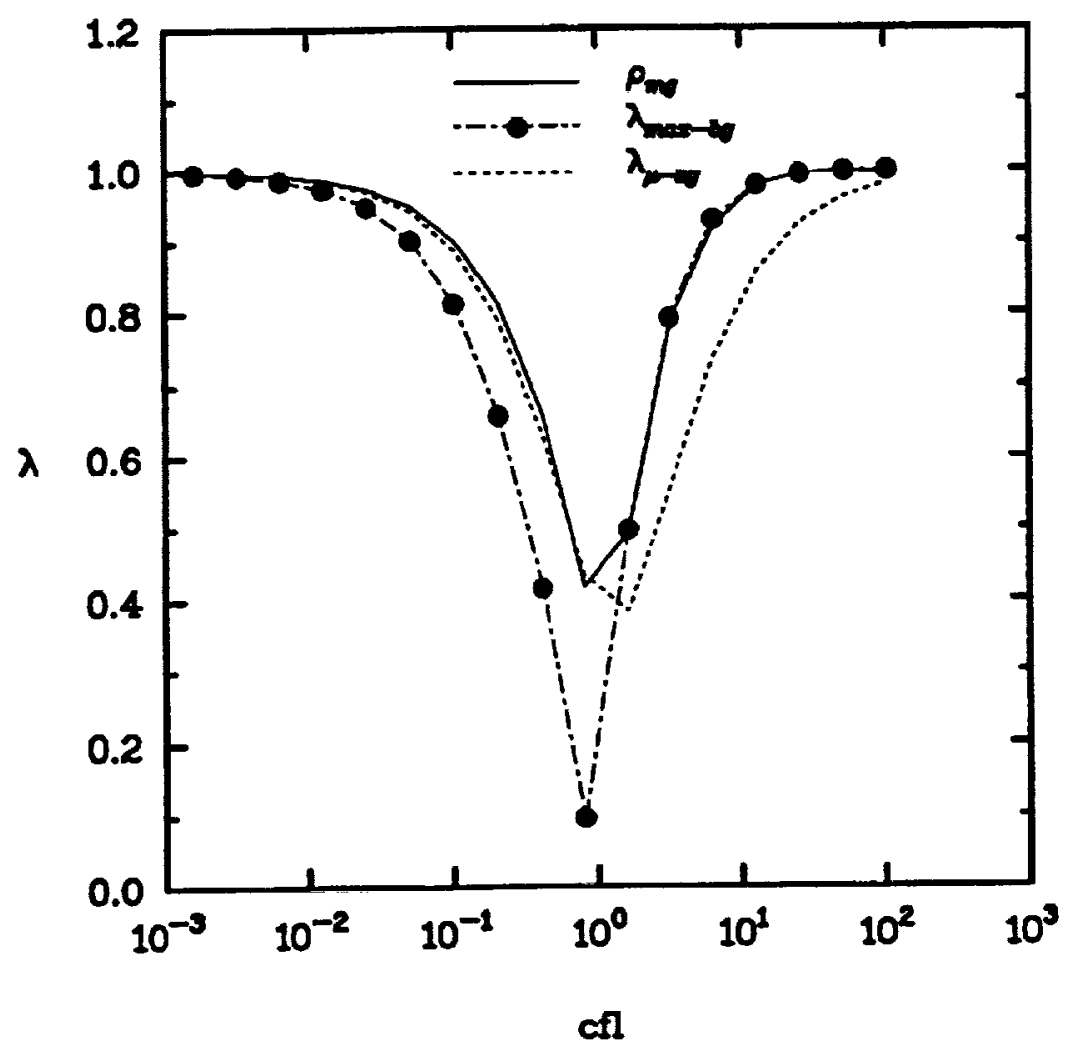

Fig. 5 : Convergence Characteristics for 1-D Convection Equation

(a) Implicit (b) Sani-implicit time integrations

(Dirichlet B.Crs) 
(a)

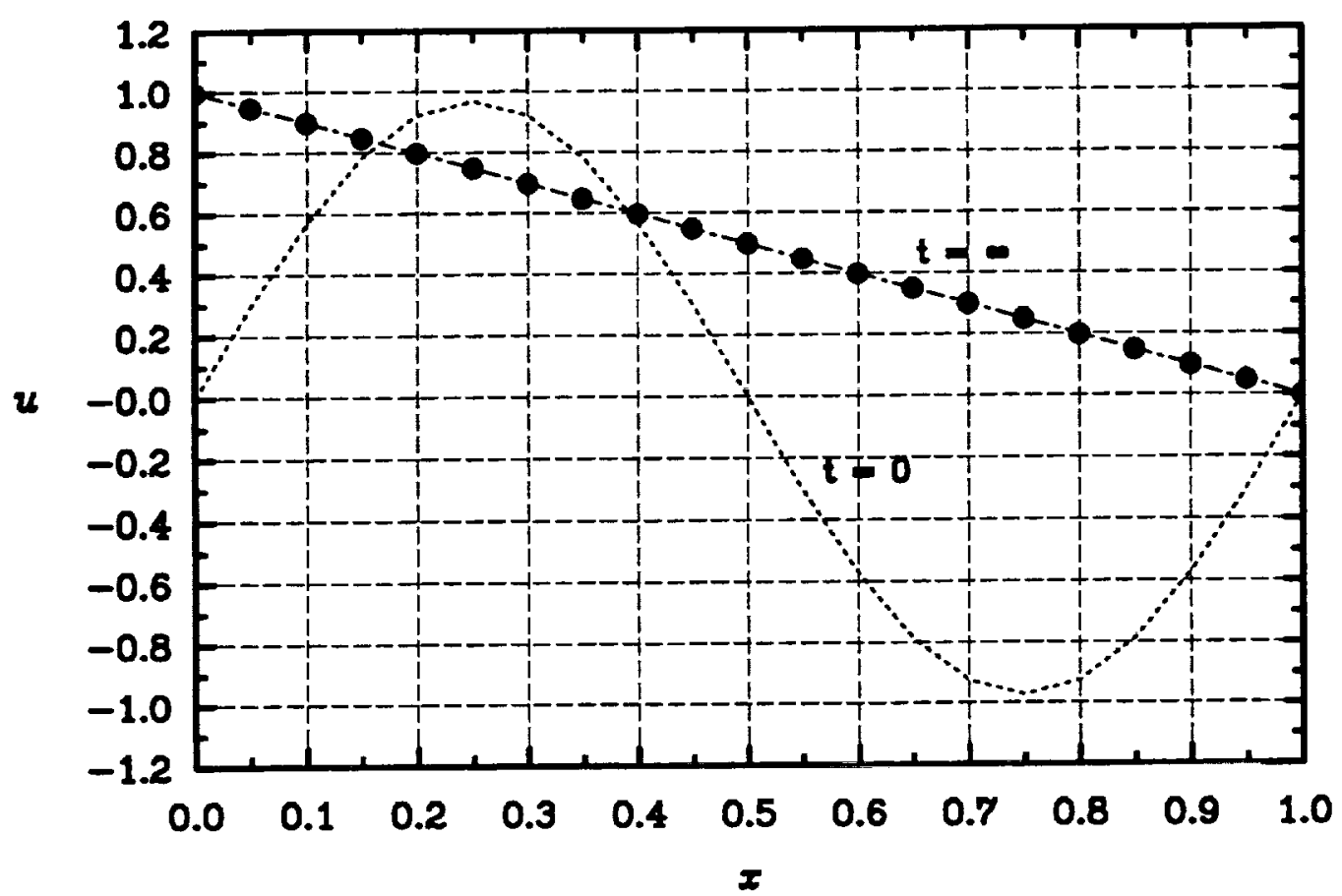

(b)

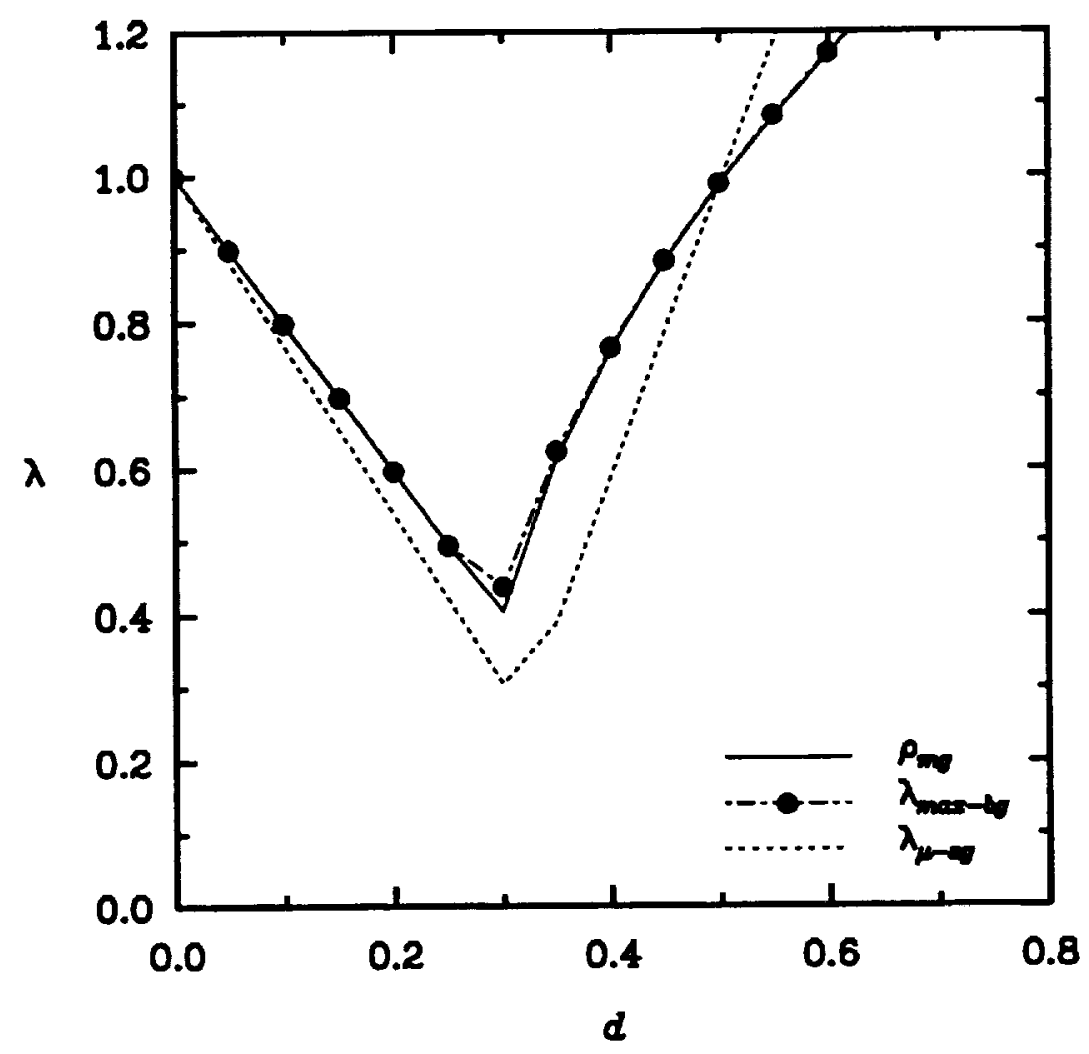

Fig. 6: 1-D Diffusion Equation (a) Stoady solution (b) Convergence Characteristics (Euler forward explicit; Dirichlet B.C's) 


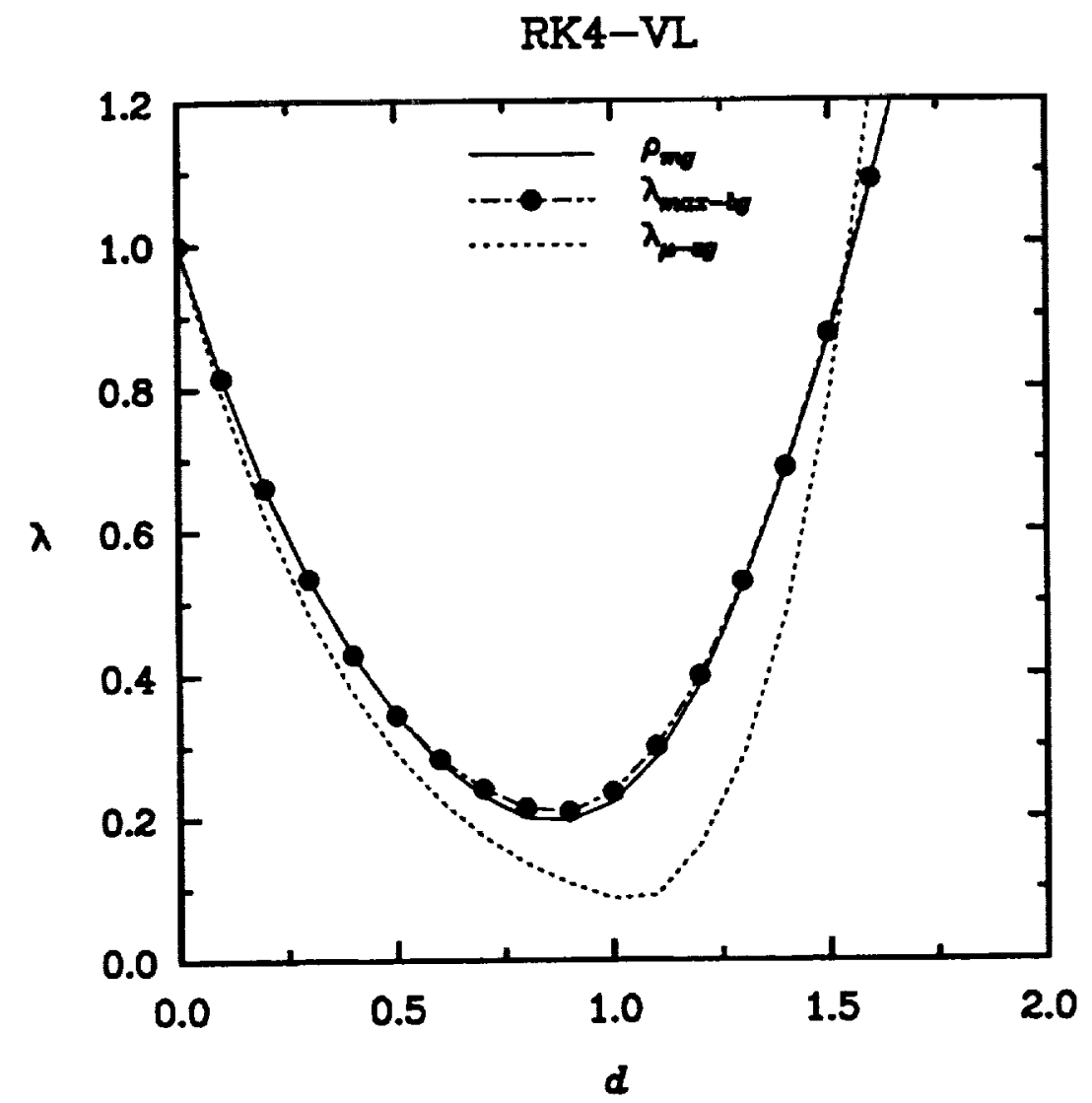

Fig. 7: Convergence Characteristics for 1-D Diffusion Equation (4-Stage Runge Kutta; Dirichlet B.C's; van Leer coefficionte) 
(a)

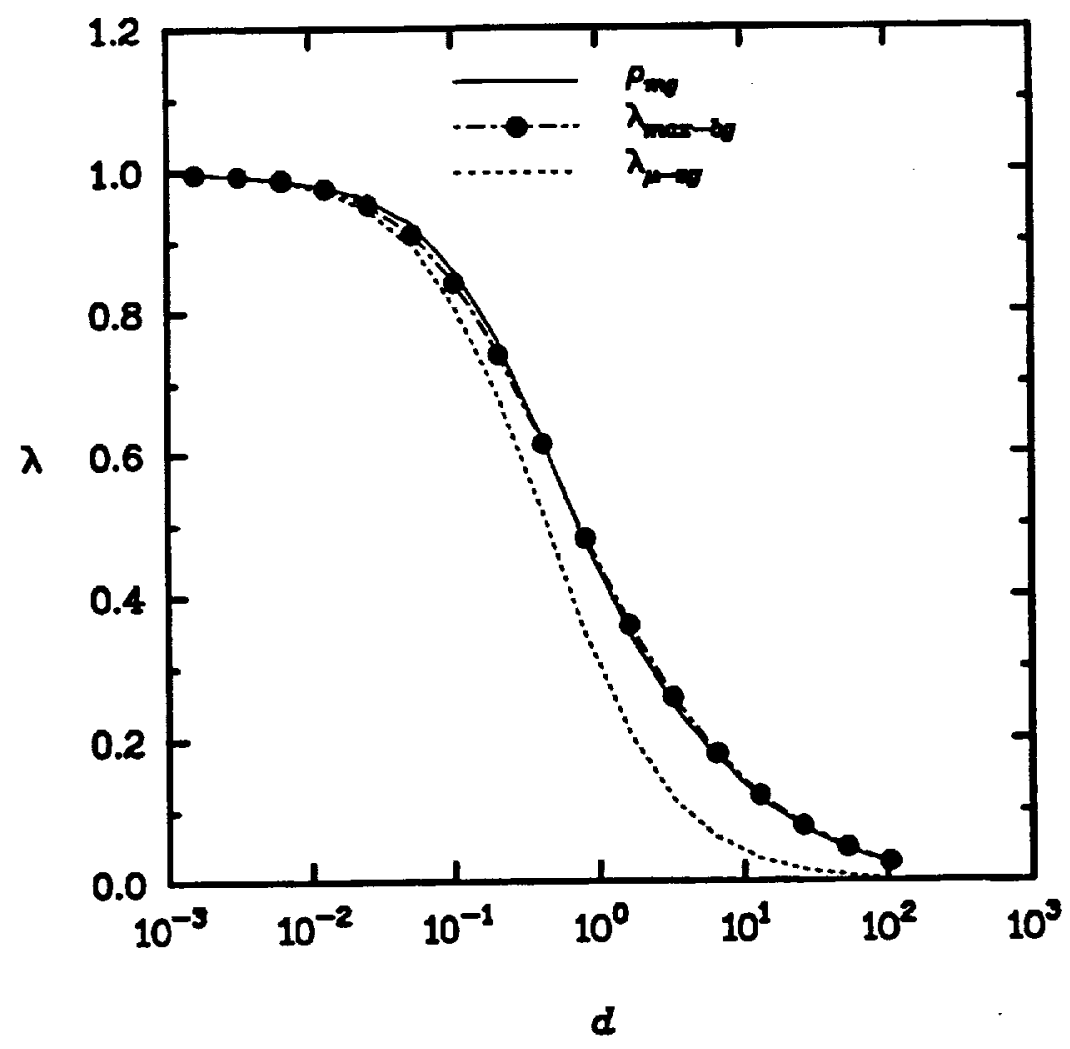

(b)

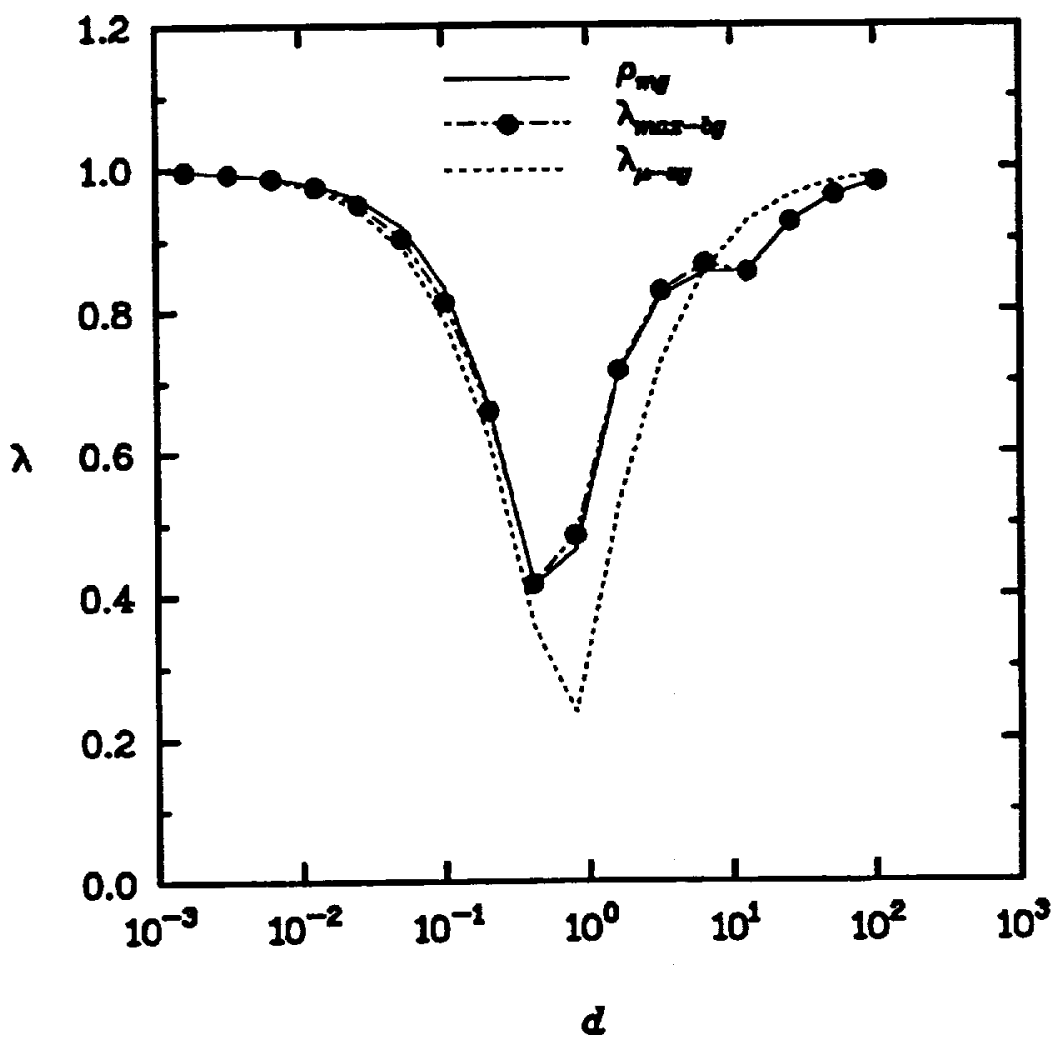

Fg. 8 : Convargence Characteristics for 1-D Diffusion Equation

(a) Implicit (b) Semi-implictt time integrations

(Dirichlet B.C's) 
(a) Variation of Peclet Number

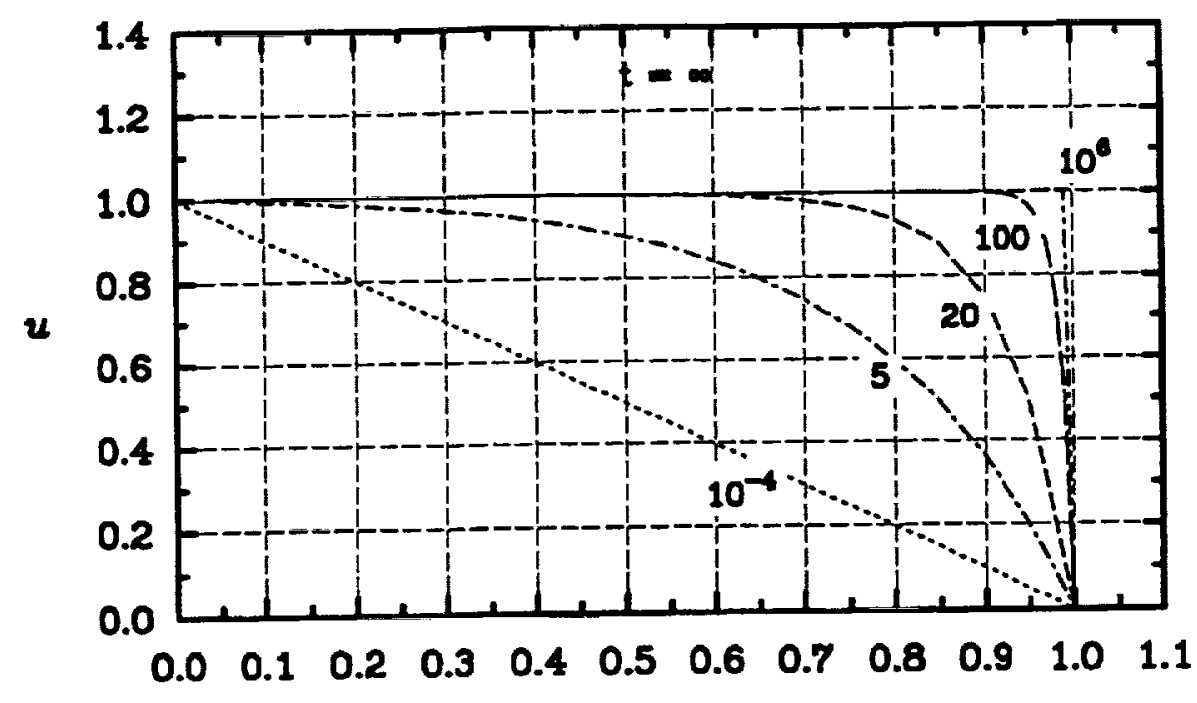

(b) $\mathrm{Pe}=10^{-4}$

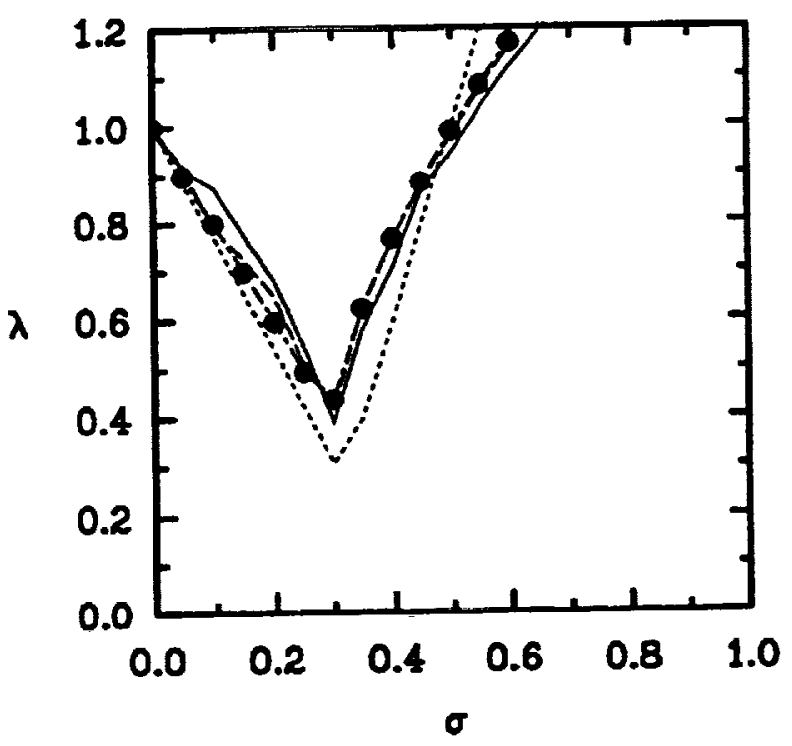

(d) $\mathrm{Pe}=100$

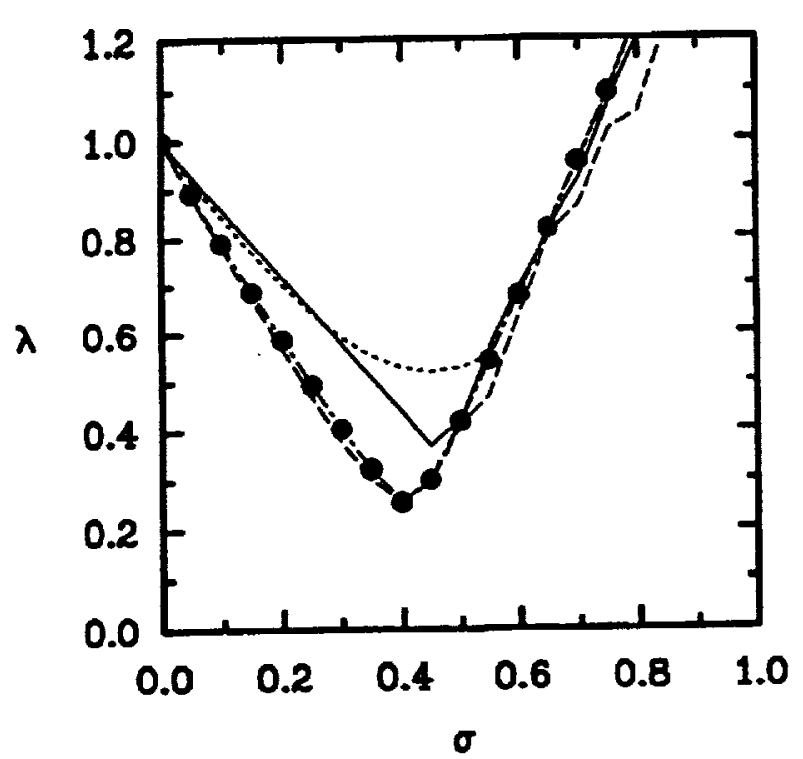

(c) $\mathrm{Pe}=20$

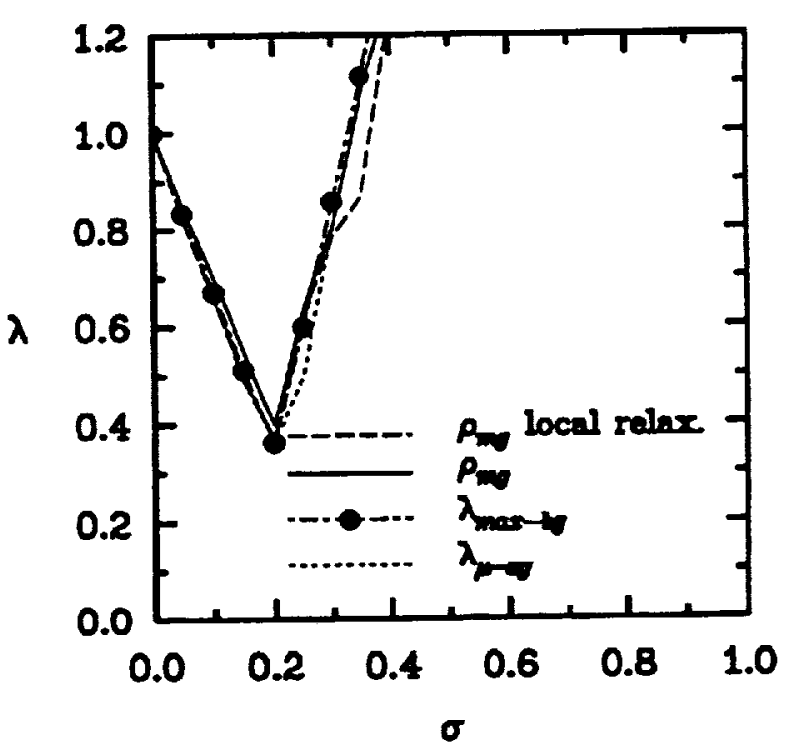

(e) $\mathrm{Pe}=10^{6}$

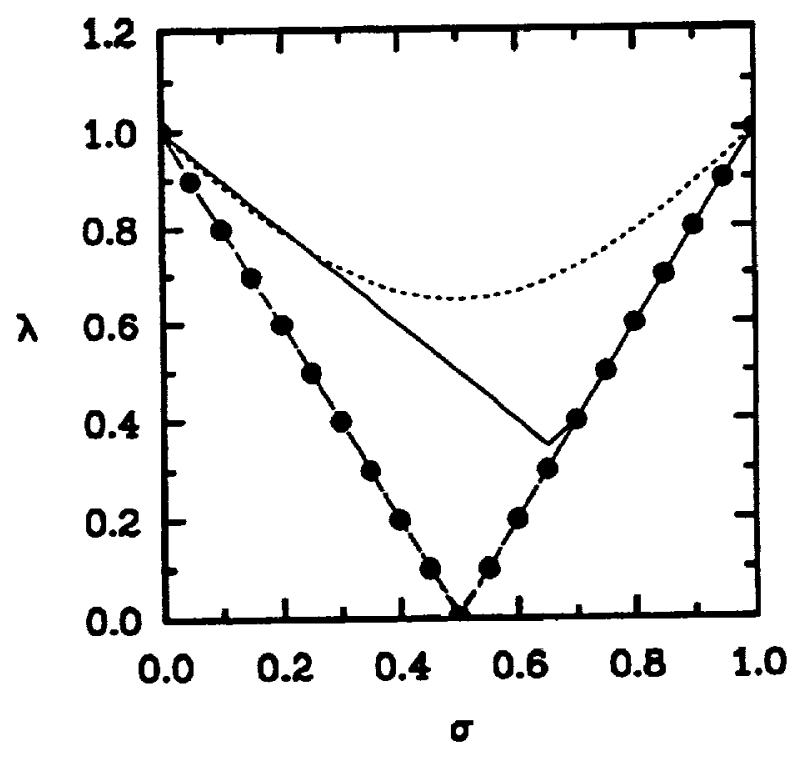

F1. 9 : 1-D Linow Burgers Equation (a) Steady Solutios, (b)-(o) Convergence Characteristics (Euler forward expliciti 1st $O$ accurate) 
(a) $\mathrm{Pe}=10^{-4}$

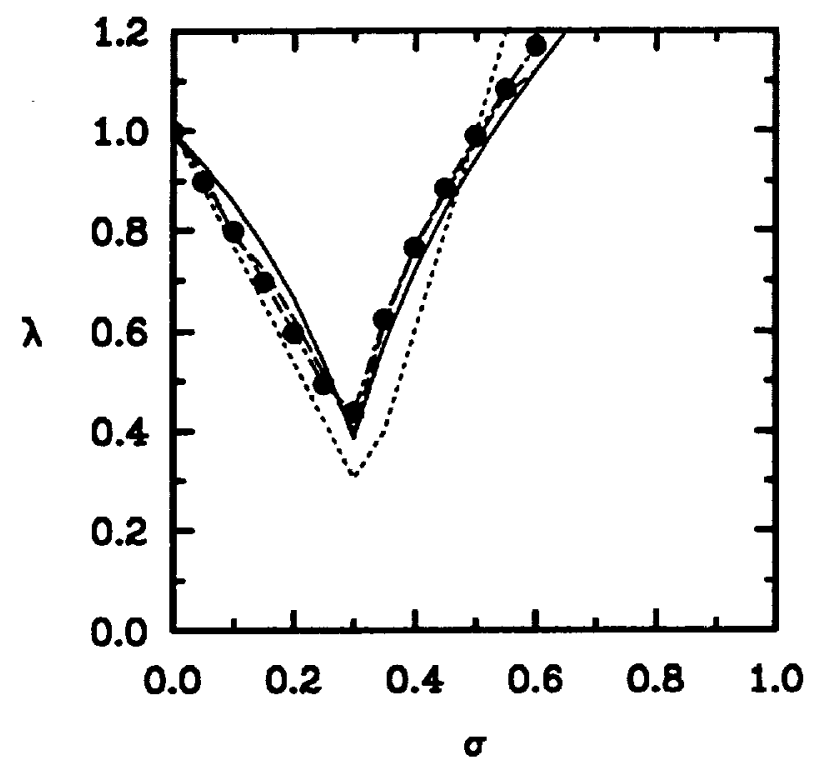

(c) $\mathrm{Pe}=100$

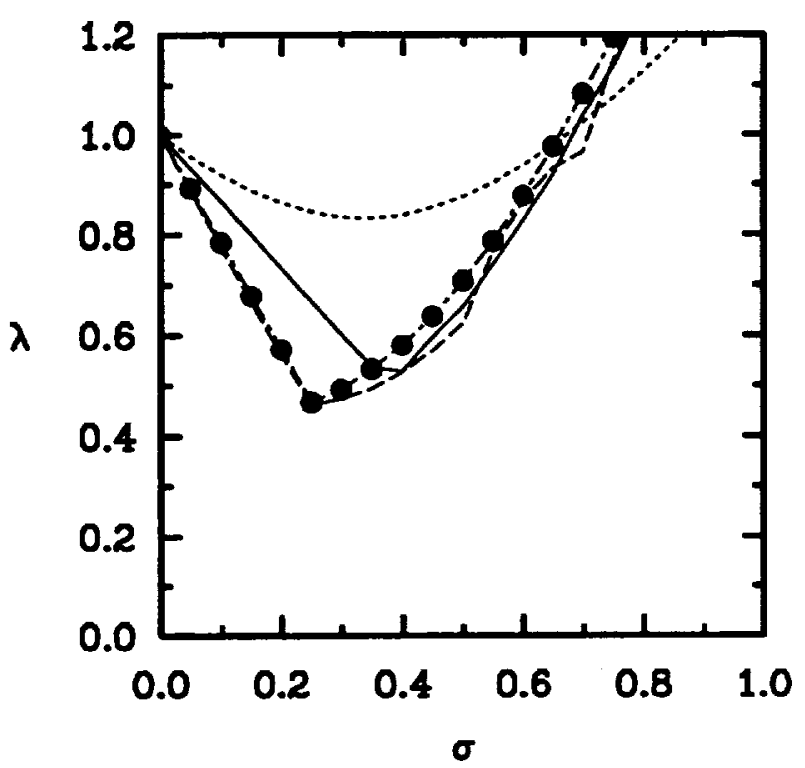

(b) $\mathrm{Pe}=20$

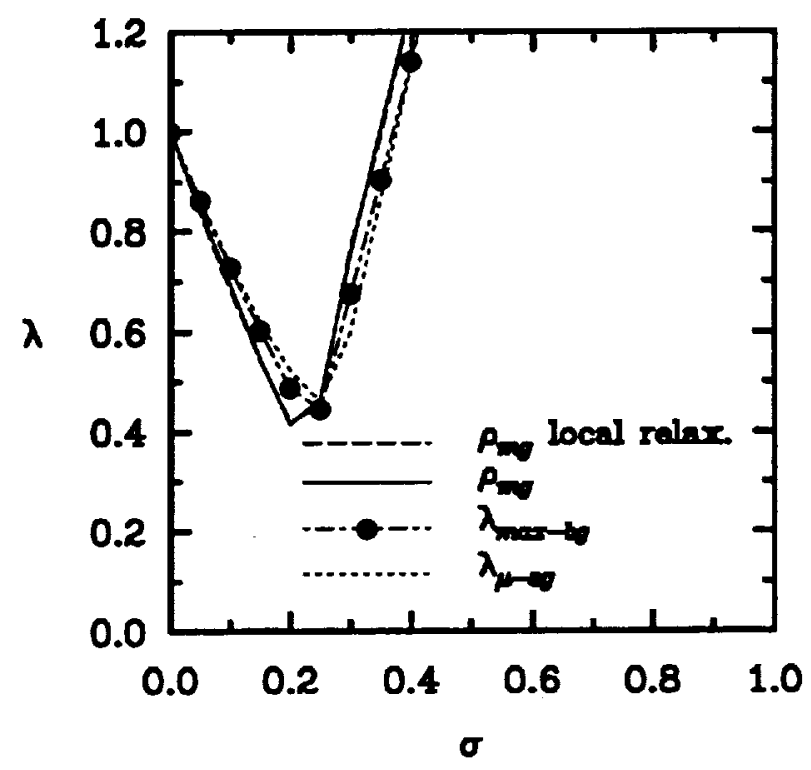

(d) $\mathrm{Pe}=10^{6}$

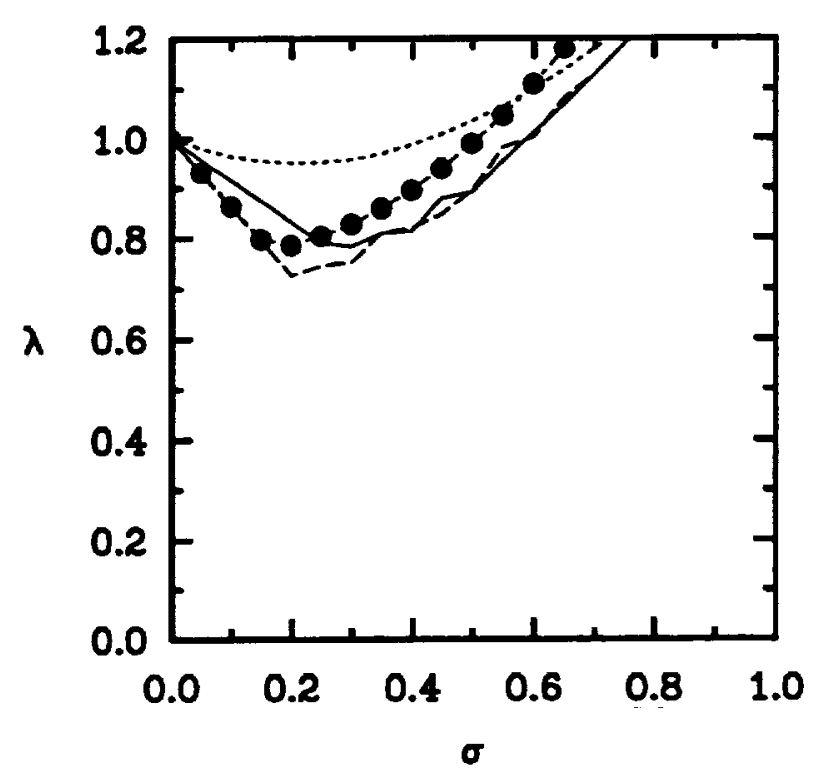

Fig. 10 : 1-D Linear Burger's Equation (a)-(d) Convergence Characteristics (Euler forward explicit; and 0 accurate) 
(a) $\mathrm{Pe}=10^{-4}$

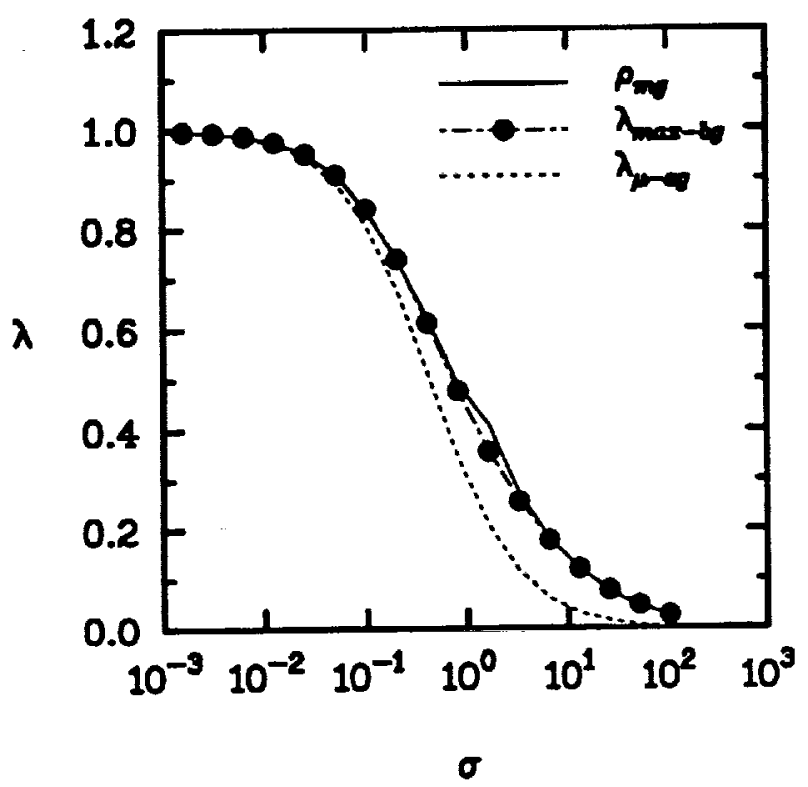

(c) $\mathrm{Pe}=100$

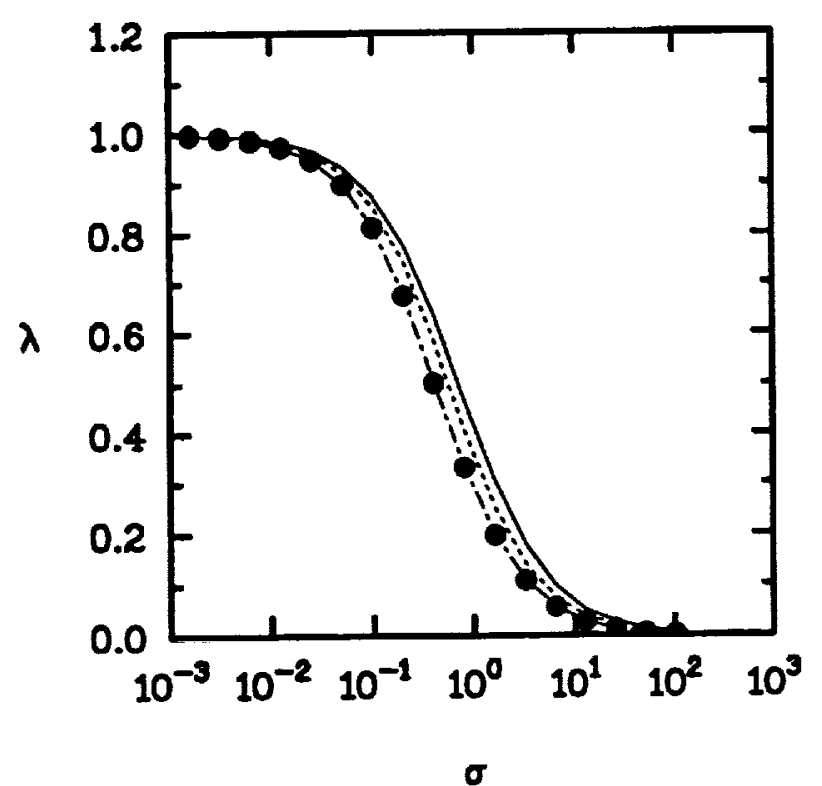

(b) $\mathrm{Pe}=20$

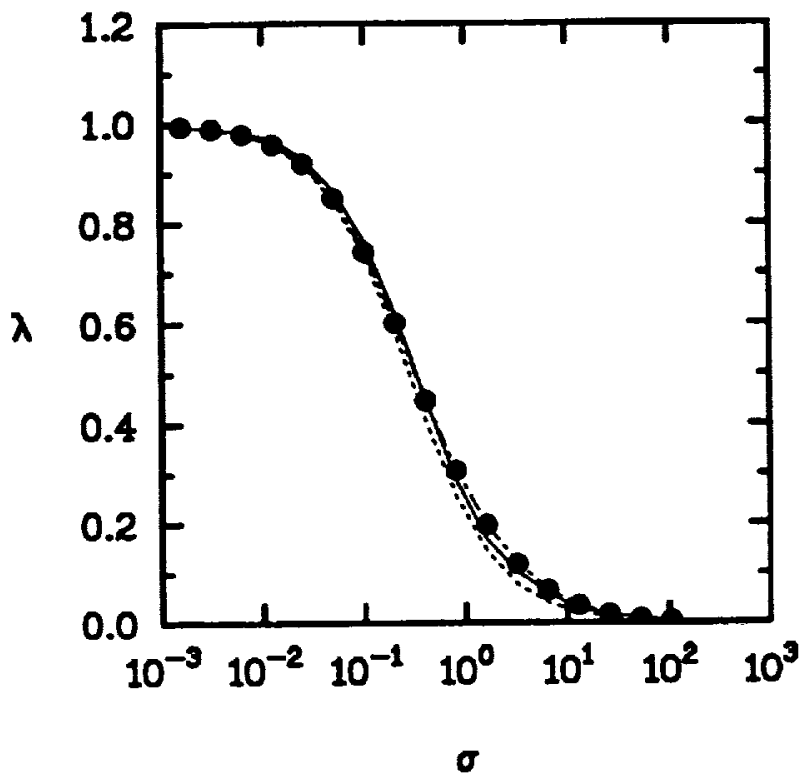

(d) $\mathrm{Pe}=10^{6}$

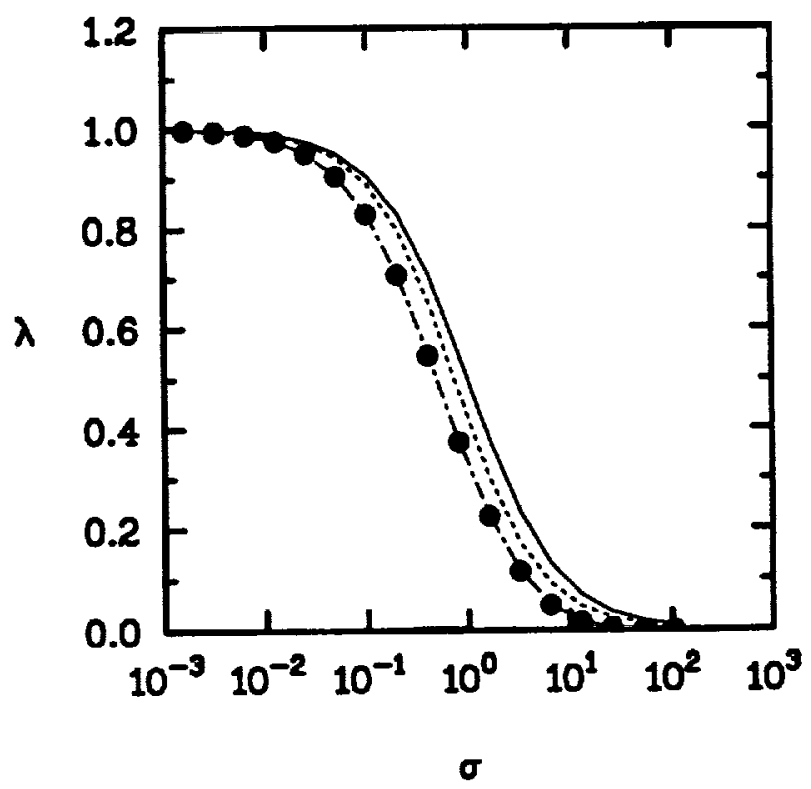

Fig. 11 : 1-D Linoar Burger's Bquation (a)-(d) Convergence Characteristica (Implicit timo intogration) 
(a) $\mathrm{Pe}=10^{-4}$

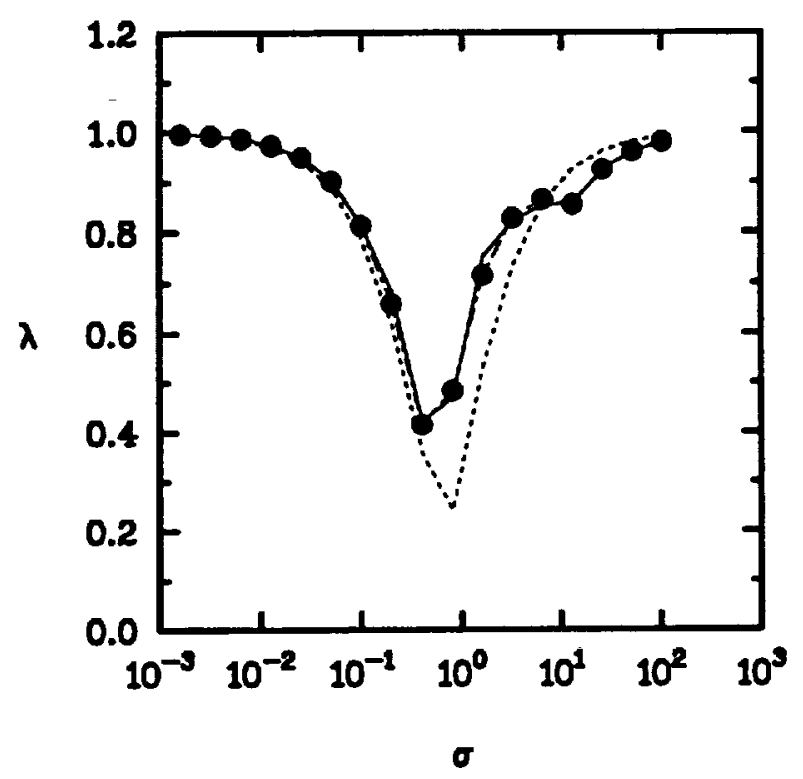

(c) $P e=100$

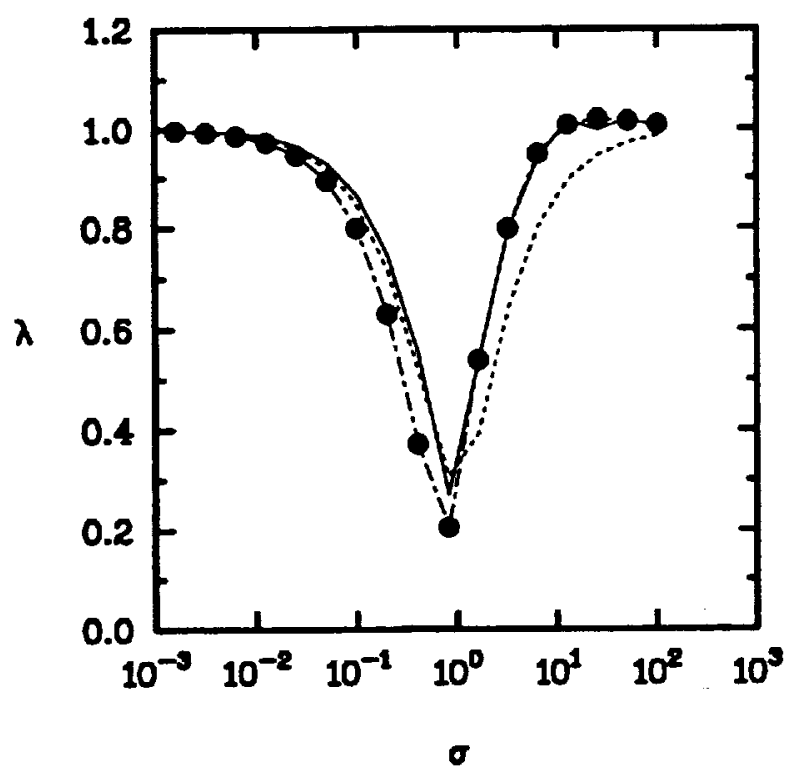

(b) $\mathrm{Pe}=20$

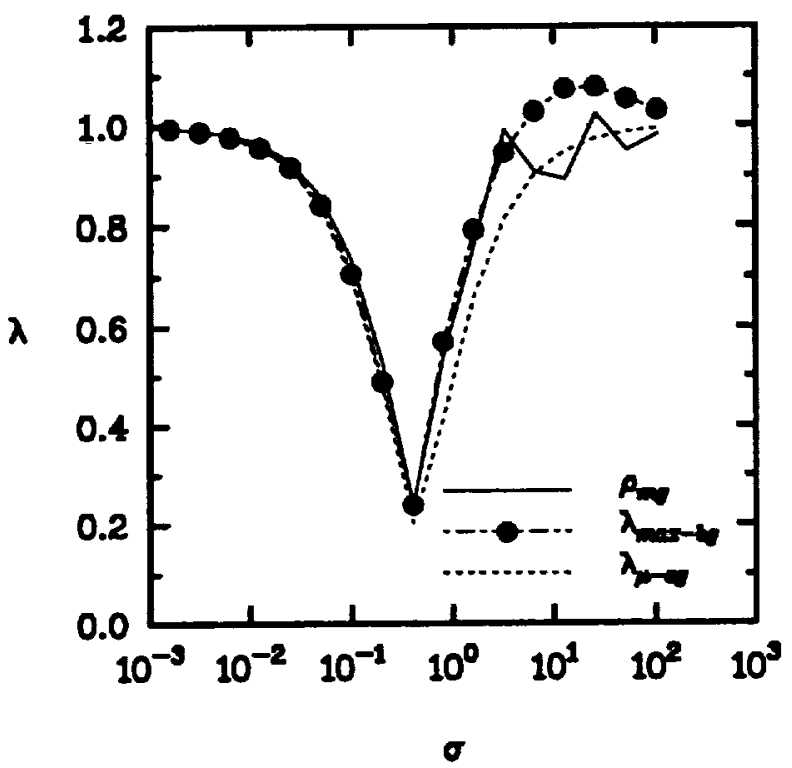

(d) $\mathrm{Pe}=10^{\circ}$

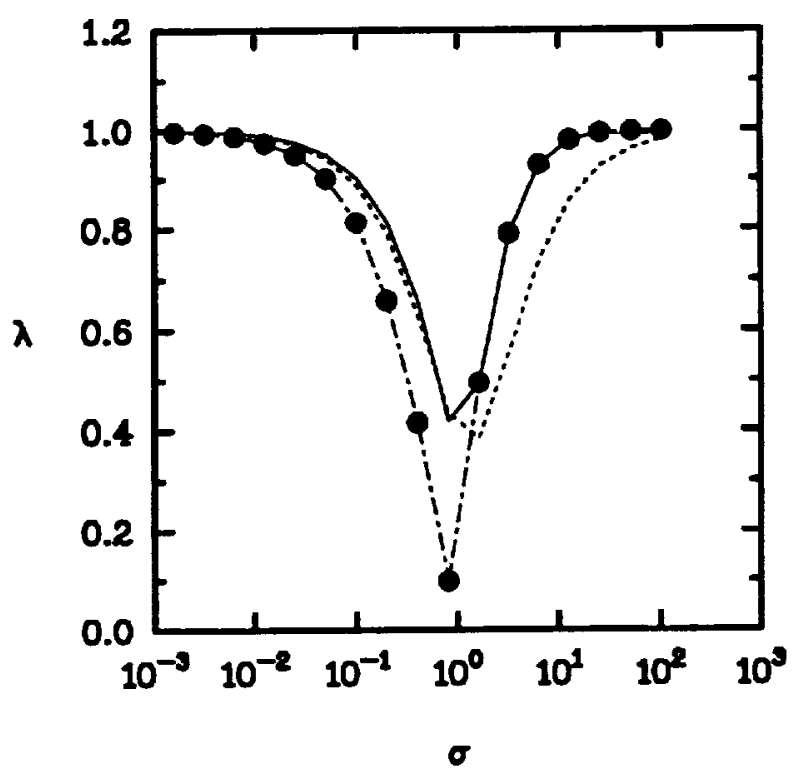

Fig. 12 : 1-D Linear Burger's Equation (a)-(d) Convergence Characteristics (Semi-implicit time integration) 


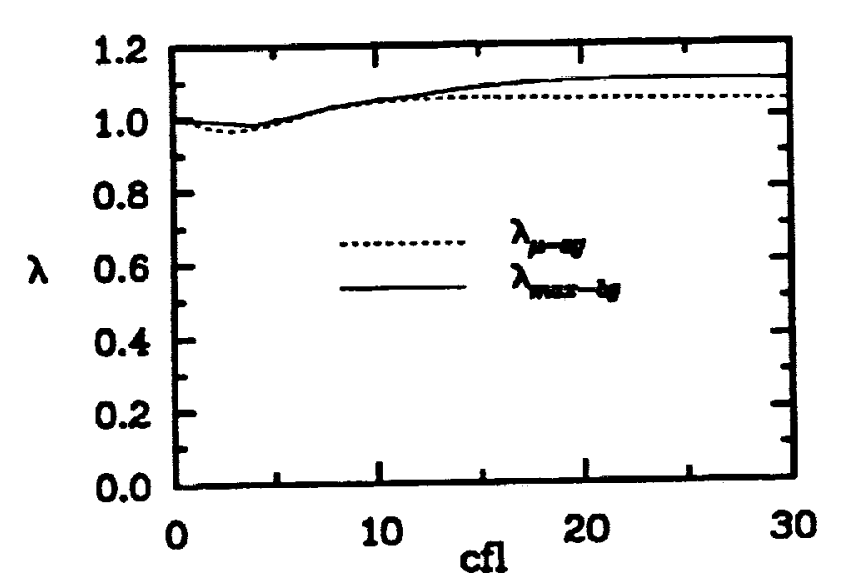

(a) Spatial Factorization

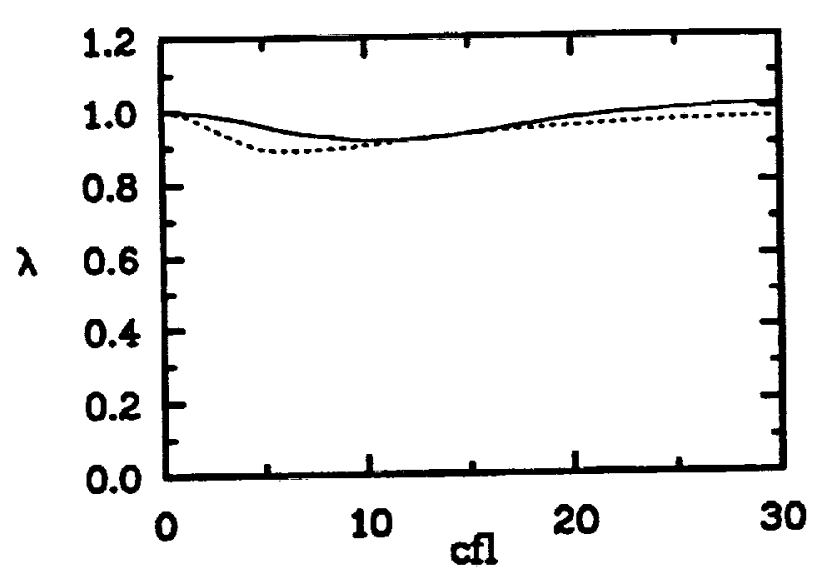

(b) Eigenvalue Factorization

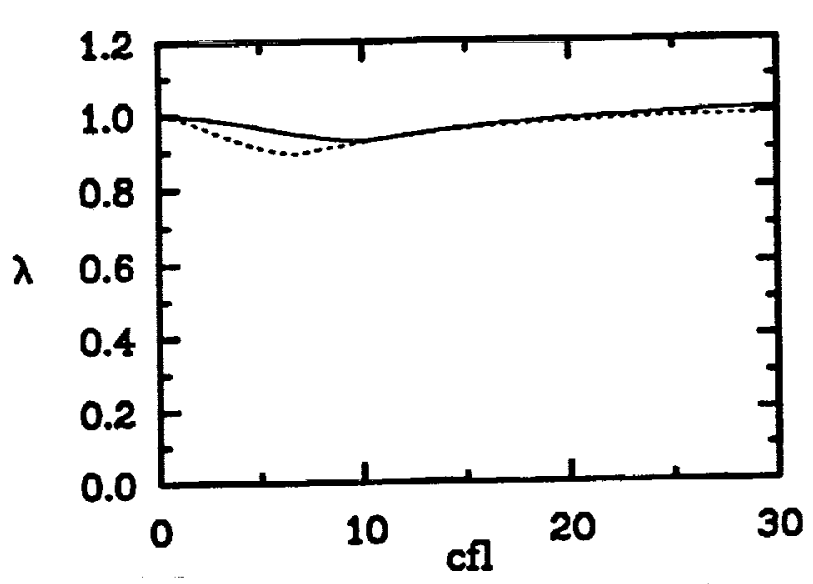

(c) Combination Factorization Steger \& Warming

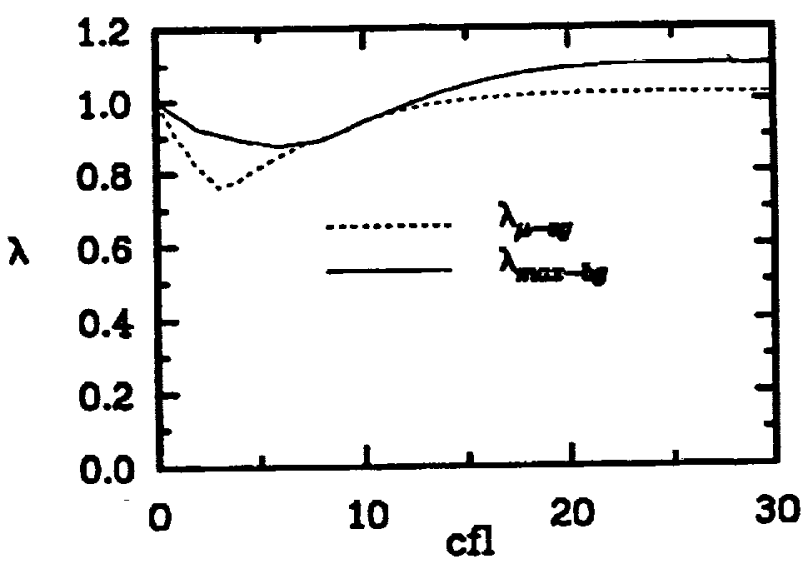

(d) Spatial Factorization

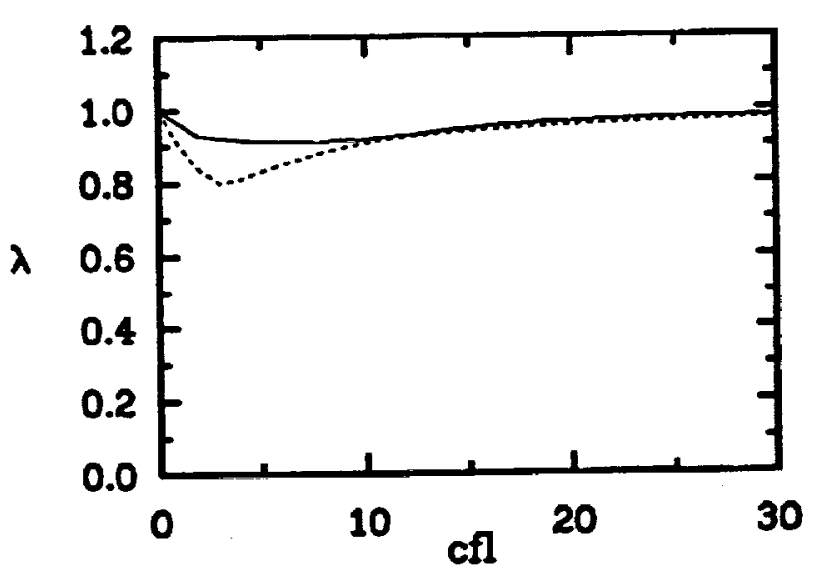

(e) Eigenvalue Factorization

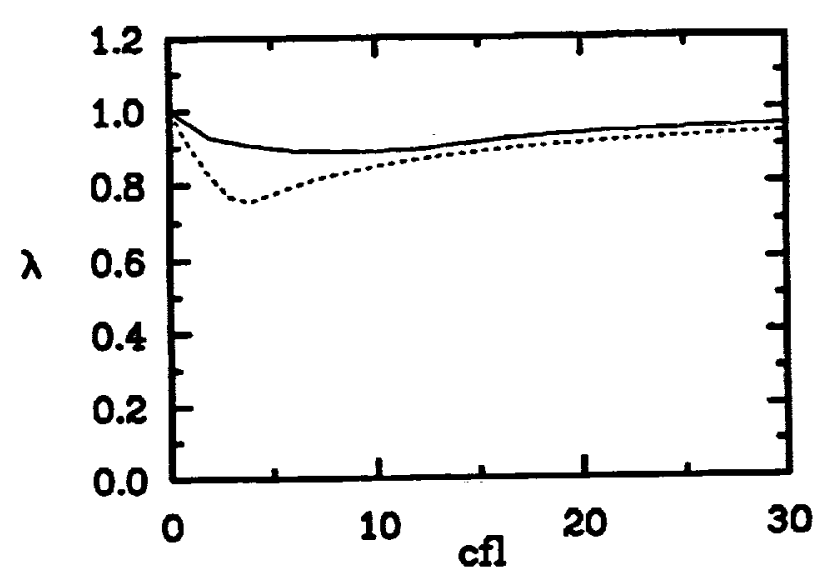

(f) Combination Factorization van Leer

Fig 13 3-D Eviler Equations using upwind schemes (a)-(f) Convergence characteristics

$$
\left(v^{2}-1 ; v^{2}-0\right)
$$




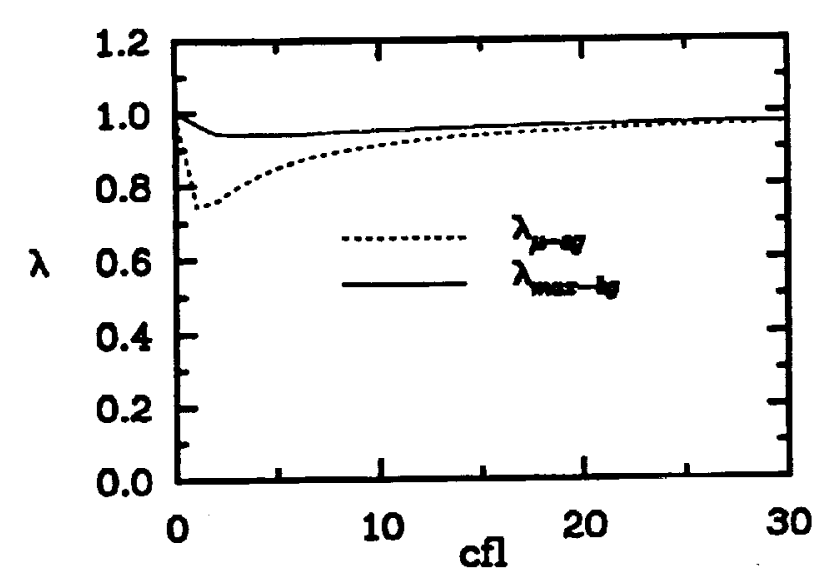

(a) $k_{2}=0, k_{4}=0.3$

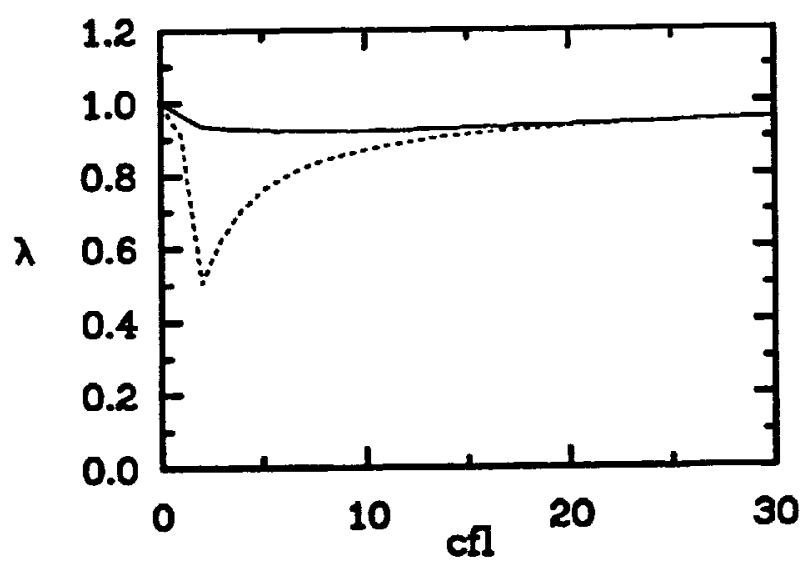

(b) $\kappa_{2}=2, \kappa_{4}=3$

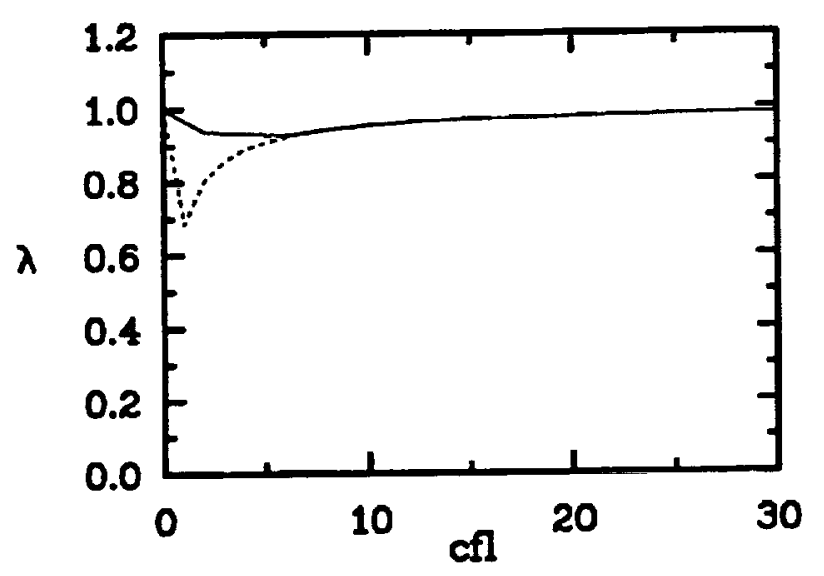

(c) $x_{2}=3, x_{4}=2$

IU Factorization

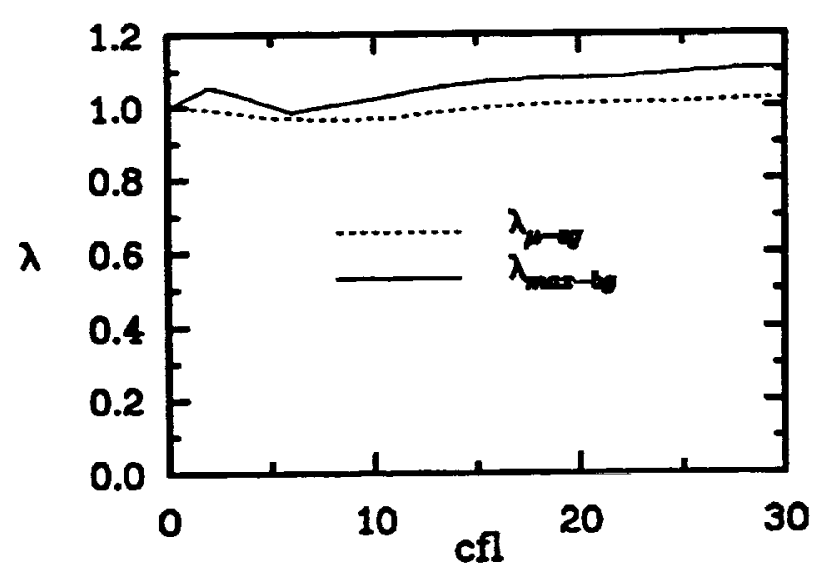

(d) $\epsilon_{i}=0, \epsilon_{i}=0$

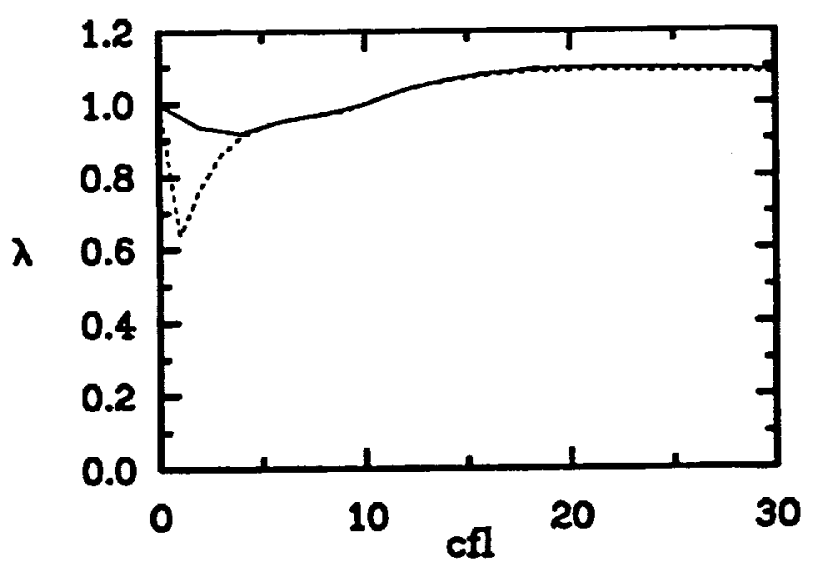

(e) $\epsilon_{e}=0.5, \epsilon_{i}=1$

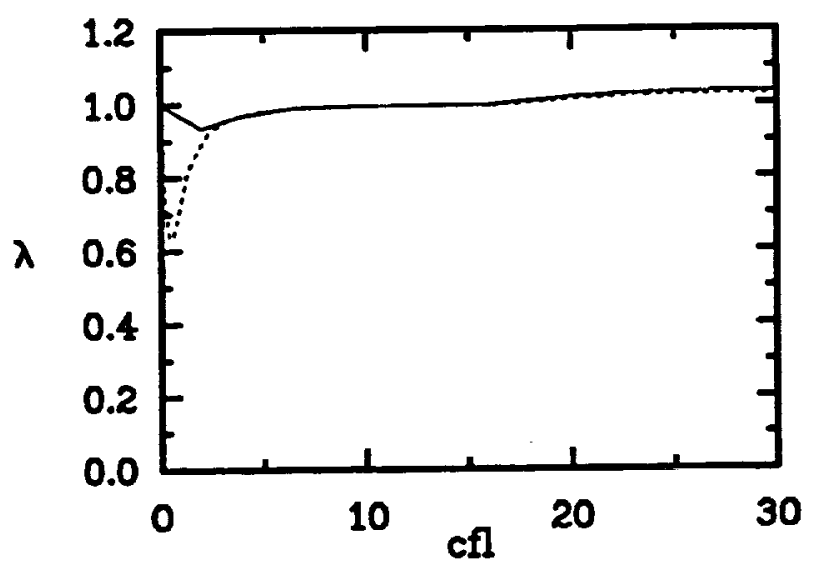

(f) $\epsilon_{i}=1, \epsilon_{i}=2$

ADI Factorization

F8. 14 3-D Euler Equations using central schomes (a)-(f) Convergence charactaristics

$$
\left(\nu^{2}=1 ; \nu^{2}=0\right)
$$



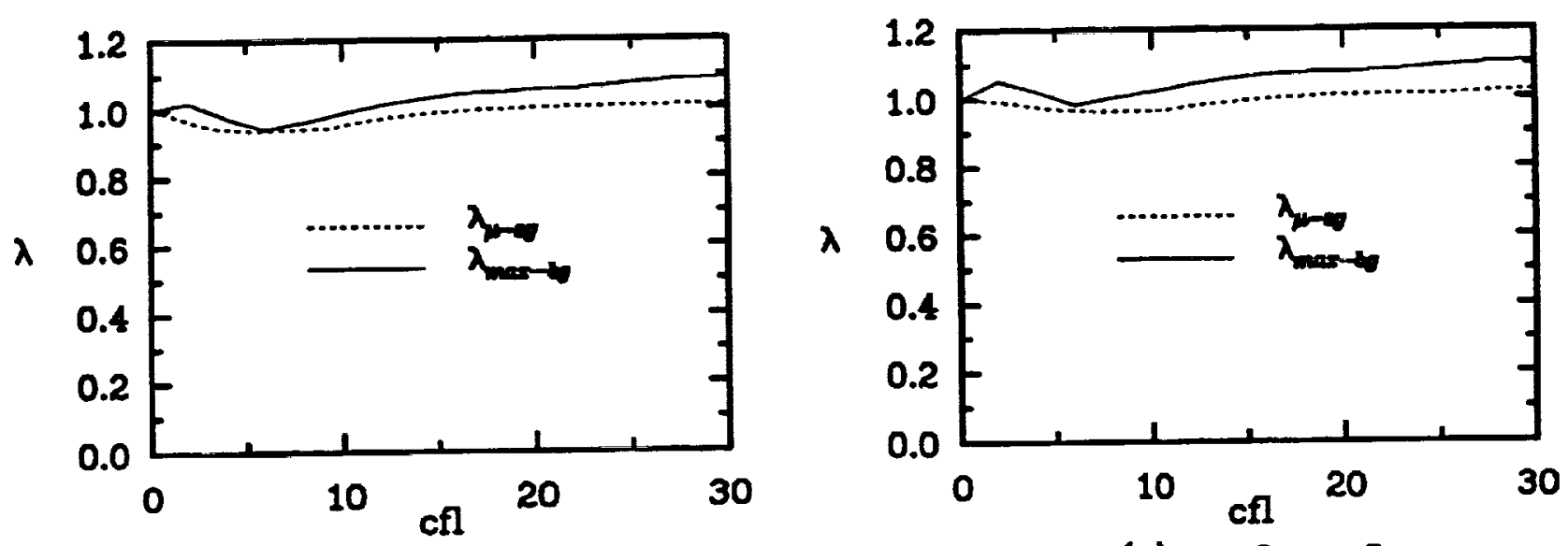

(a) $\epsilon_{c}=0, \epsilon_{q}=0$
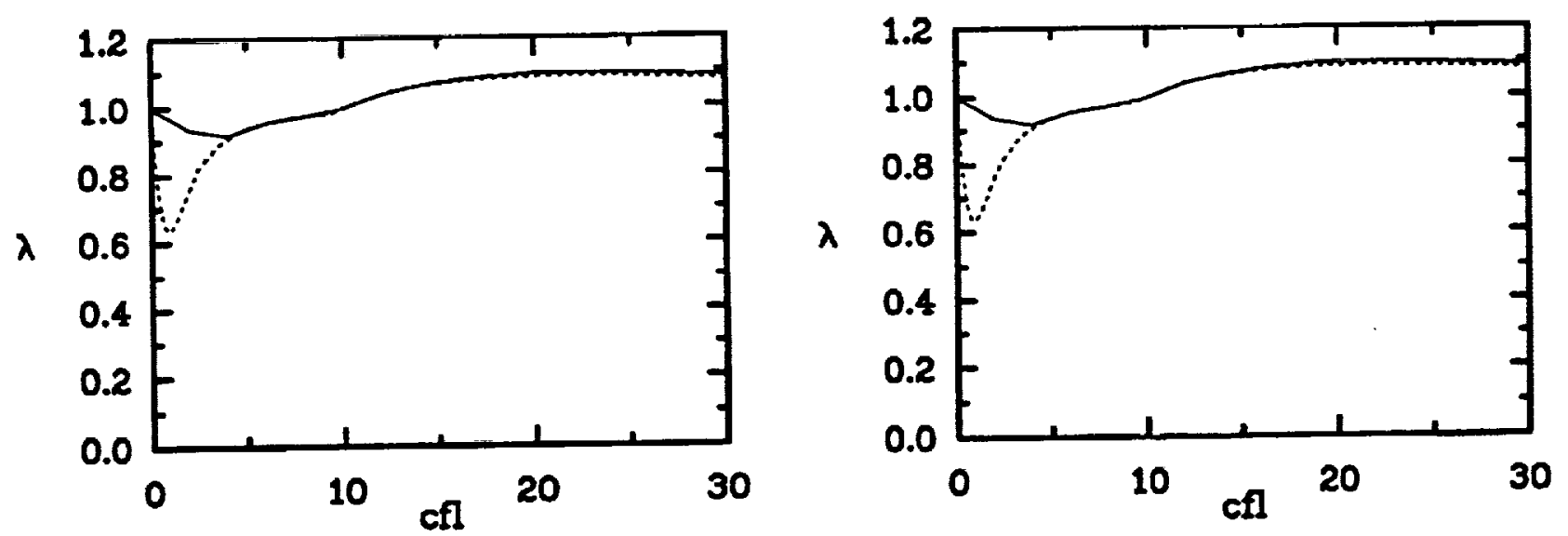

(b) $\varepsilon_{q}=0.5, \varepsilon_{t}=1$

(e) $\epsilon_{e}=0.5, \epsilon_{t}=1$
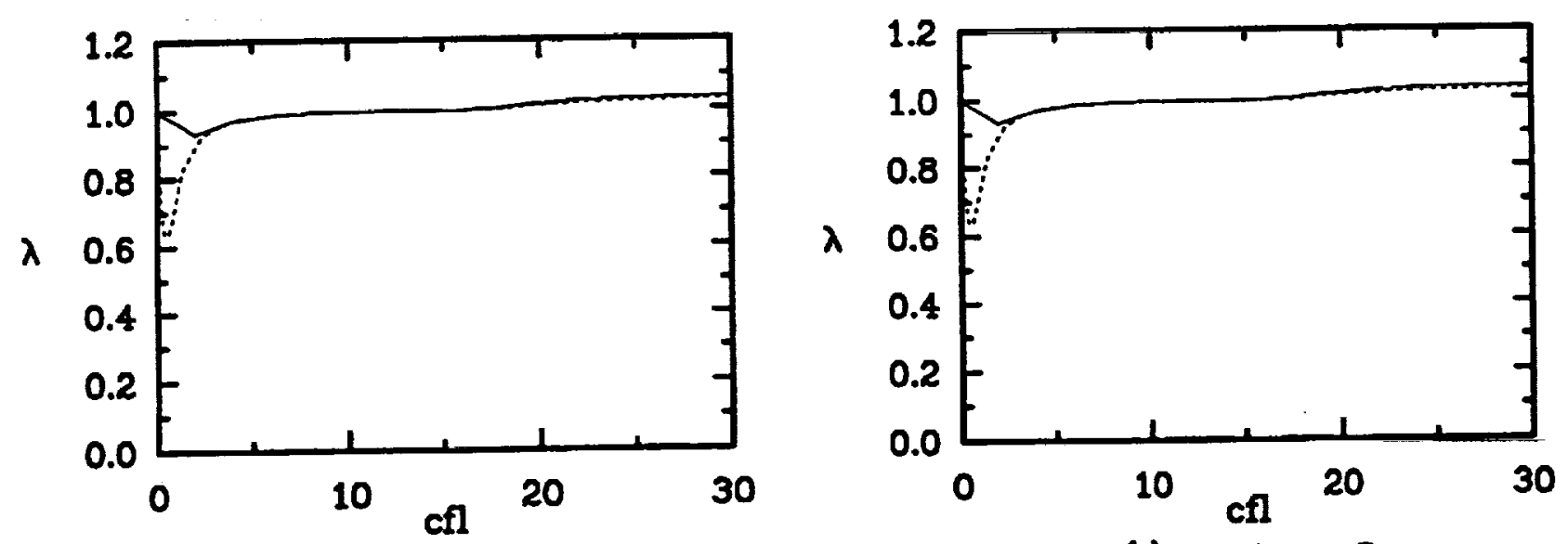

(c) $\epsilon_{a}=1, \epsilon_{i}=2$

(f) $\varepsilon_{i}=1, \varepsilon_{i}=2$

$$
\text { Re }=10^{2}
$$$$
\operatorname{Re}=10^{\circ}
$$

F3. 15 3-D Neviar-Stakes Equations using central echomes (a)-(f) Convergence charactaristics $\left(v^{2}=1 ; v^{2}=0\right)$ 


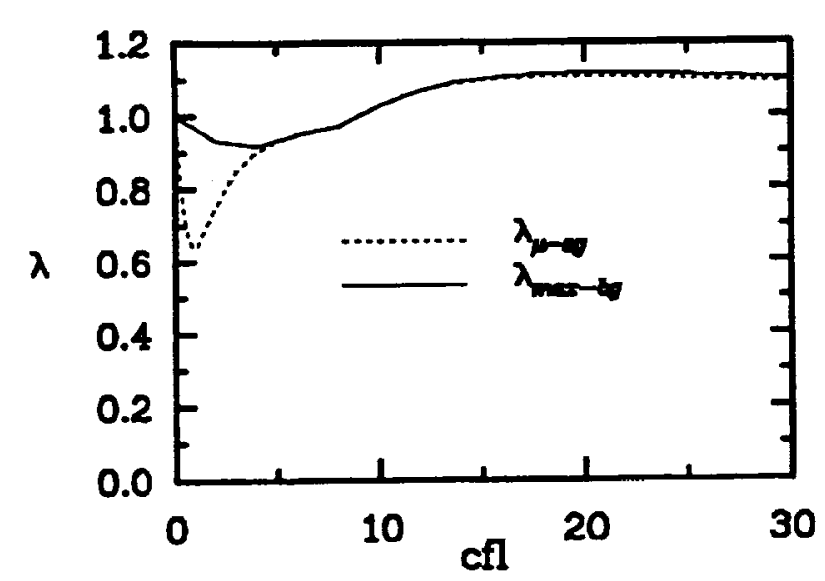

(a) $\alpha_{y}=15^{\circ}, \alpha_{a}=0^{\circ}$

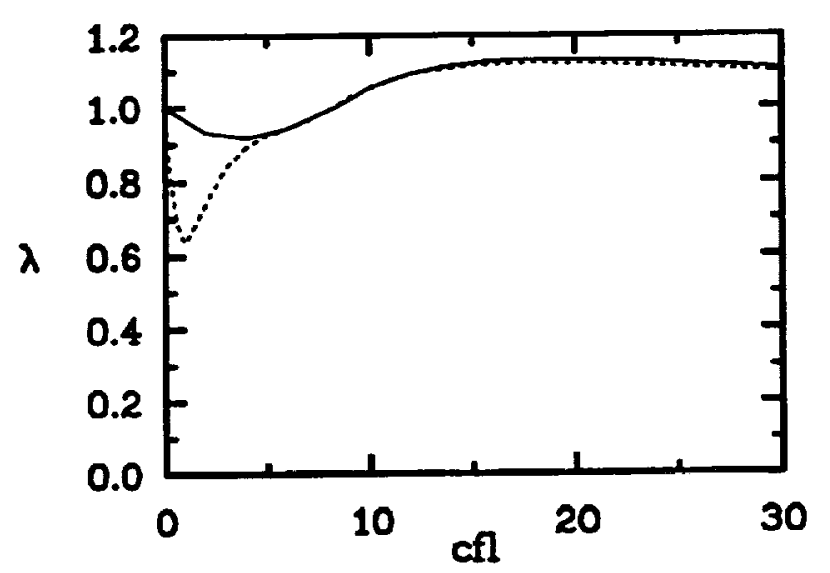

(b) $\alpha_{\nu}=45^{\circ}, \alpha_{a}=0^{\circ}$

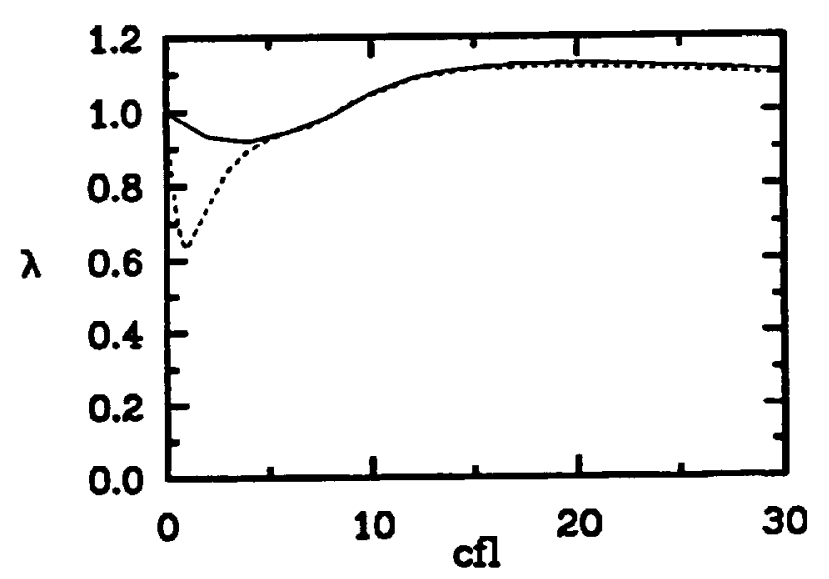

(c) $\alpha_{y}=60^{\circ}, \alpha_{a}=0^{\circ}$

Yaw Angle

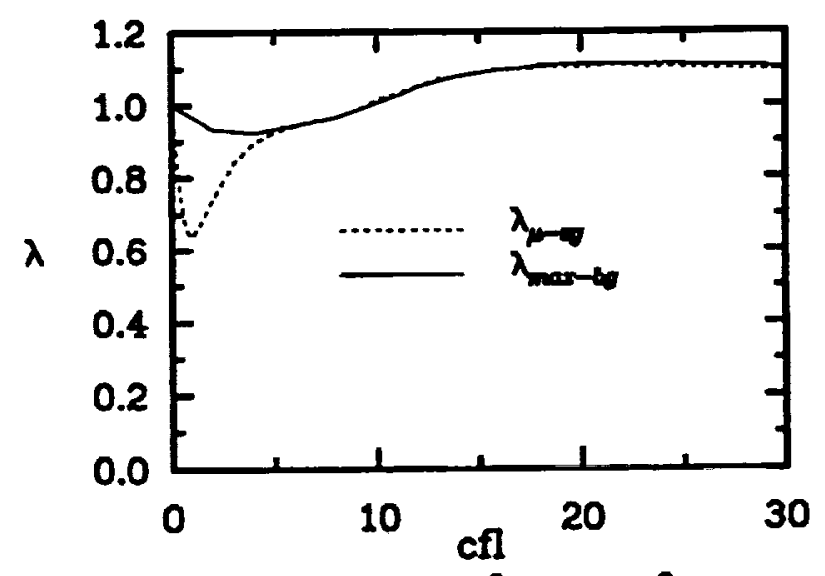

(d) $\alpha_{\nu}=15^{\circ}, \alpha_{\alpha}=15^{\circ}$

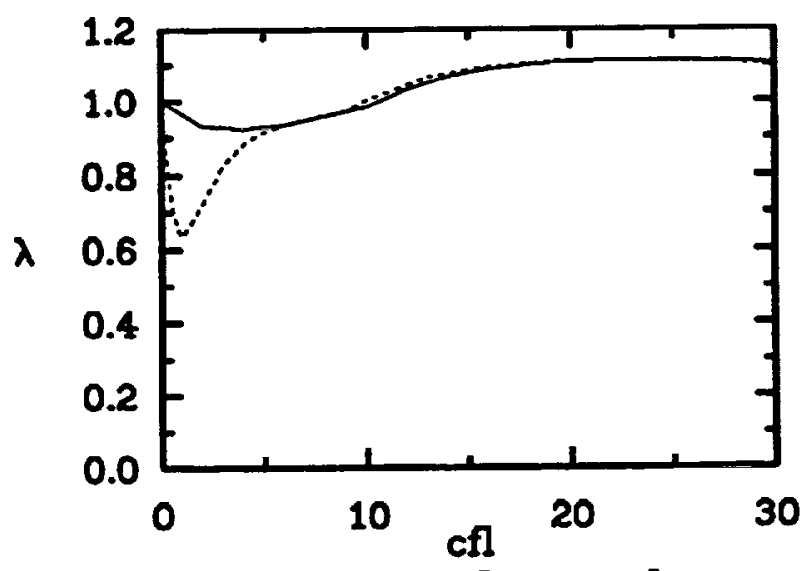

(e) $\alpha_{y}=45^{\circ}, \alpha_{a}=45^{\circ}$

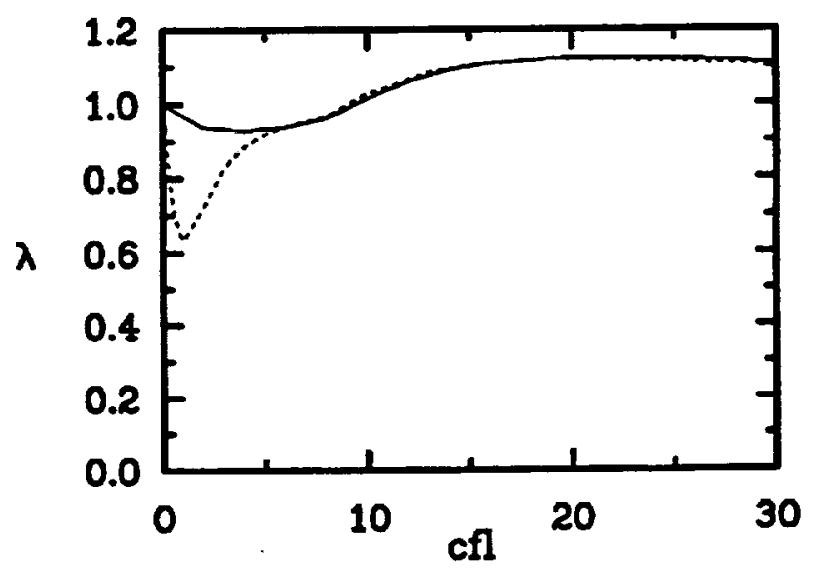

(f) $\alpha_{y}=60^{\circ}, \alpha_{a}=60^{\circ}$

Yaw and Angle of Attack

Fig 16 3-D Navier-Stokes Equations using central schomes (a)-(f) Convergence charactaristics $\left(R e=100, c_{0}=0.5, c_{1}-1.0, v^{1}=1, v^{2}=0\right)$ 


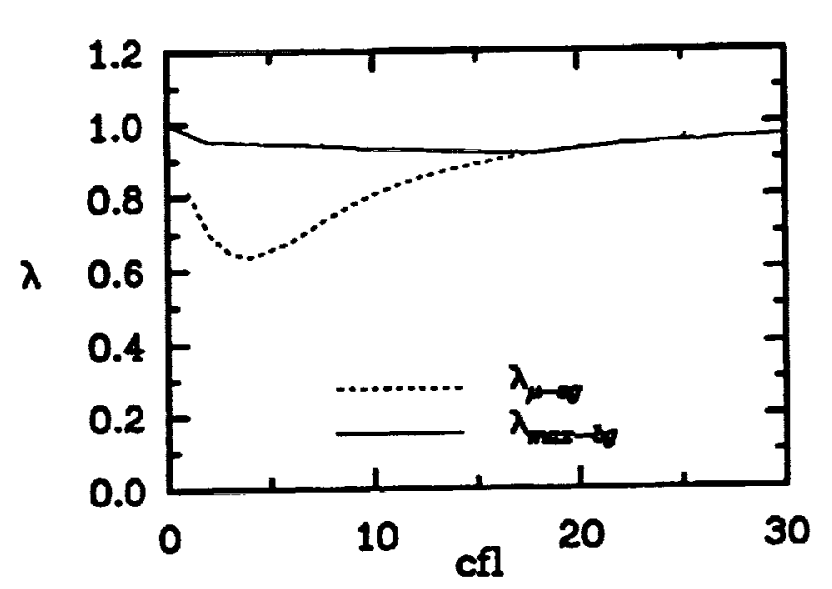

(a) $\Delta x / \Delta y=10, \Delta x / \Delta z=1$

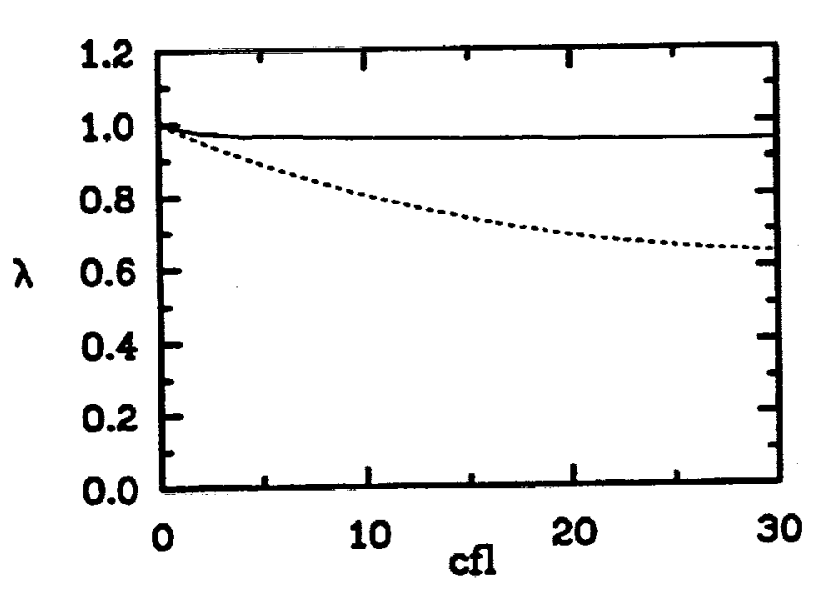

(b) $\Delta x / \Delta y=100, \Delta x / \Delta z=1$

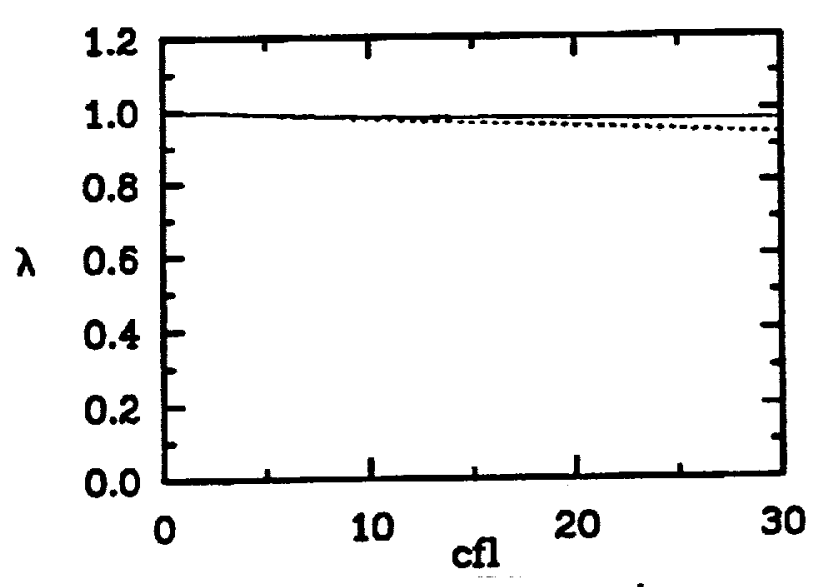

(c) $\Delta x / \Delta y=1000, \Delta x / \Delta z=1$

Aspect Ratio

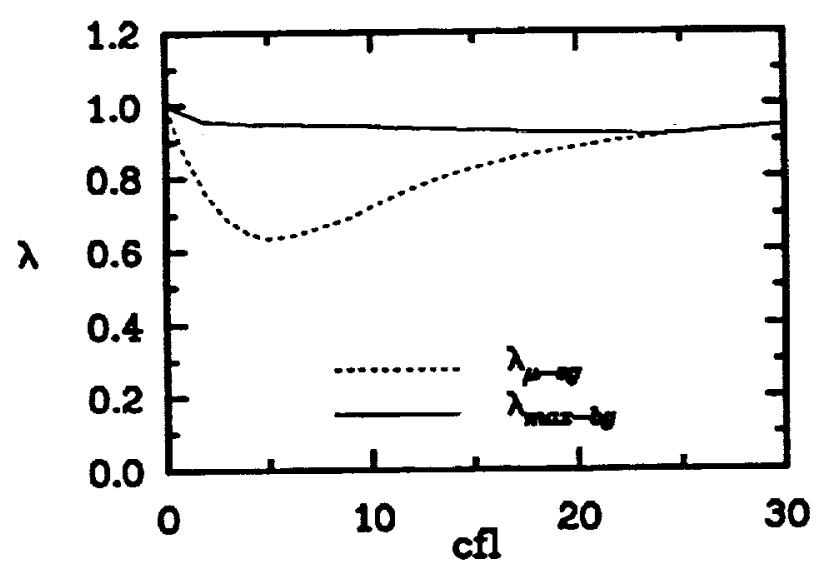

(d) $\Delta x / \Delta y=\Delta x / \Delta z=10$

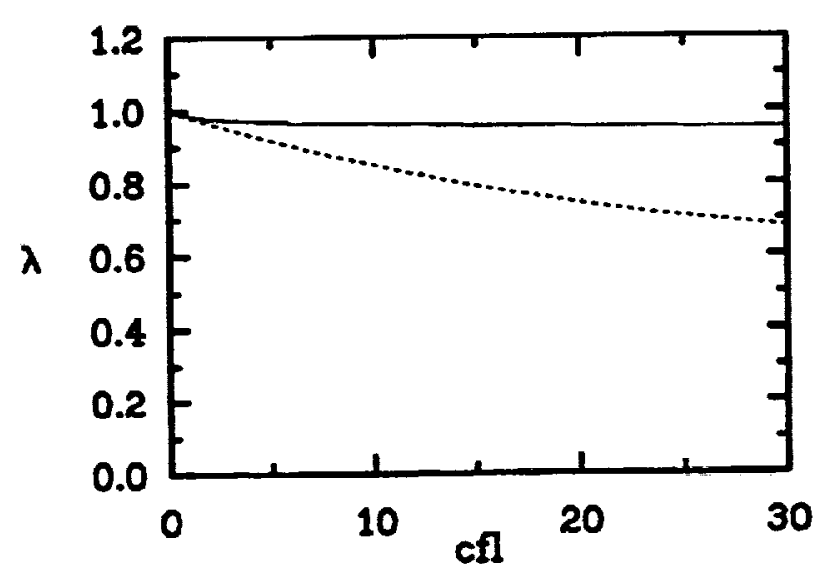

(e) $\Delta x / \Delta y=\Delta x / \Delta z=100$

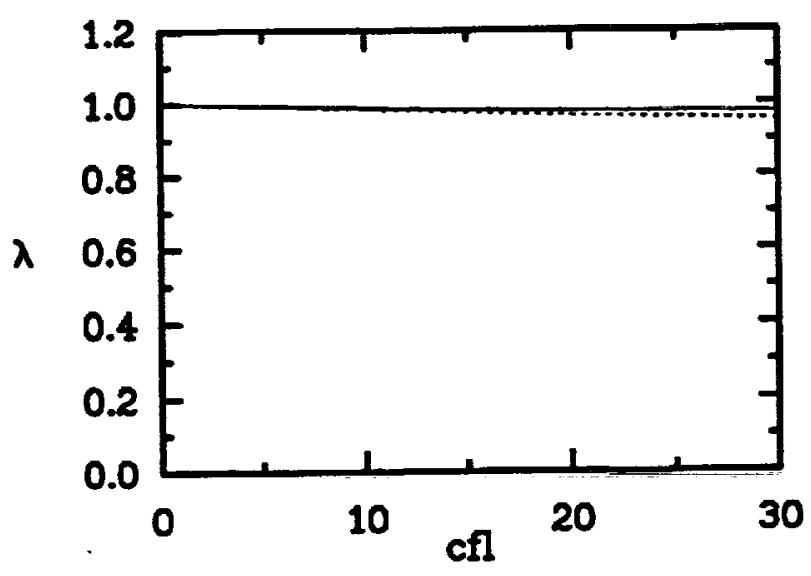

(f) $\Delta x / \Delta y=\Delta x / \Delta z=1000$

Aspect Ratio

Fig 17 3-D Navier-Stokes Equations using central schames (a)-(f) Convergence charactariatics $\left(R_{0}=100, \varepsilon_{0}=0.5, \varepsilon^{-10}, v^{2}-1, v^{2}=0\right)$ 


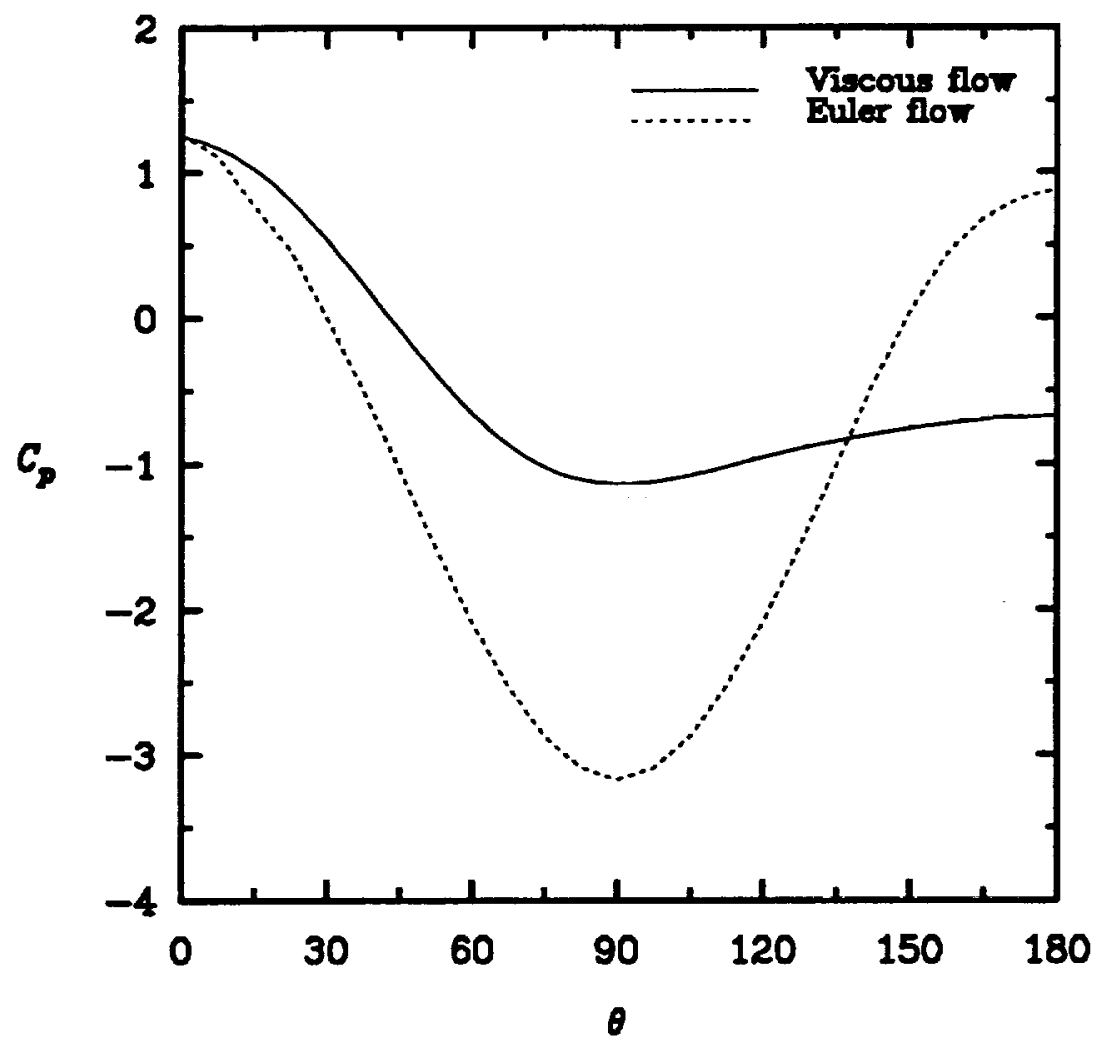

(a) Pressure coefficients for flow past a circular cylinder

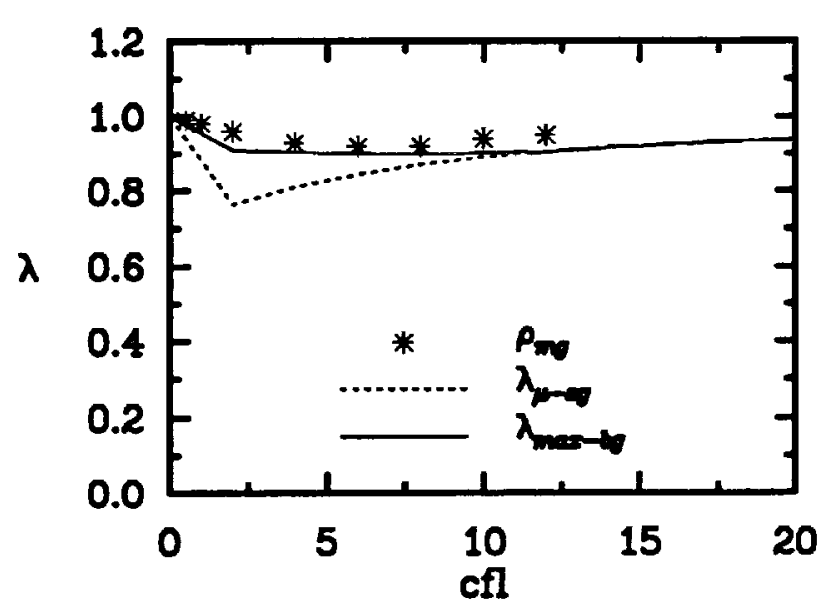

(b) Inviscid flow

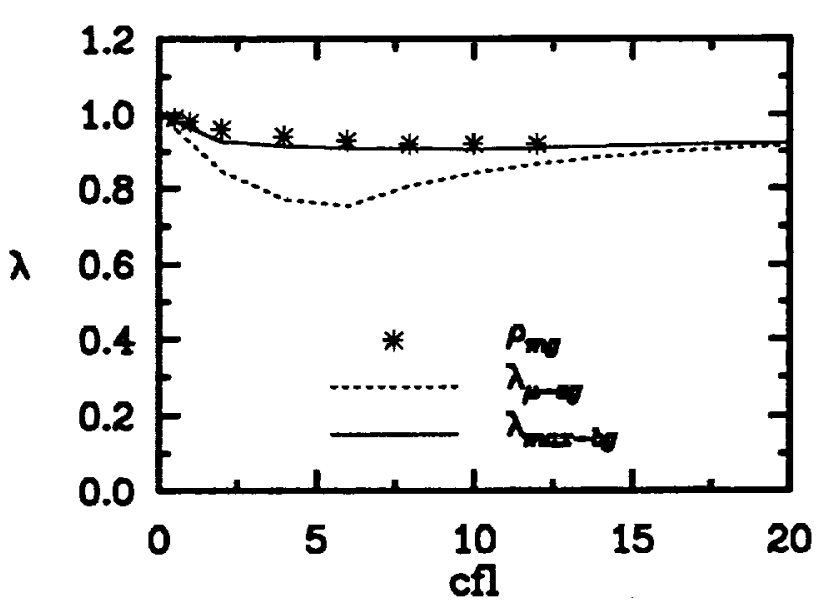

(c) Viscous flow

Fig. 18 2-D Euler and Navier-Stokes flows around a circular cylinder using ADI scheme

$$
\left(v^{2}=1, v^{2}=0, \epsilon_{0}=1, \epsilon_{i}=2\right)
$$


Public reporting burden for this collection of information is estimated to average 1 hour per response, including the time lor reviewing instructions, searching existing data sources. gathering and maintaining the data needed, and completing and reviewing the collection of intormation. Send comments regarding this burden estimate or any other aspect of this collection of information, including suggestions for reducing this burden, to Washington Headquarters Services, Directorate tor Iniormation Operations and Reports, 1215 Jetter

\begin{tabular}{|l|c|c|}
\hline 1. AGENCY USE ONLY (Leave blank) & $\begin{array}{c}\text { 2. REPORT DATE } \\
\text { October } 1994\end{array}$ & $\begin{array}{r}\text { 3. REPORT TYPE AND DATES COVERED } \\
\text { Technical Memorandum }\end{array}$ \\
\hline
\end{tabular}

\section{TITLE AND SUBTITLE}

On Bi-Grid Local Mode Analysis of Solution Techniques for 3-D Euler and Navier-Stokes Equations

6. AUTHOR(S)

S.O. Ibraheem and A.O. Demuren

\section{PERFORMING ORGANIZATION NAME(S) AND ADDRESS(ES)}

National Aeronautics and Space Administration

Lewis Research Center

Cleveland, Ohio 44135-3191

9. SPONSORING/MONITORING AGENCY NAME(S) AND ADDRESS(ES)

National Aeronautics and Space Administration

Washington, D.C. 20546-0001
5. FUNDING NUMBERS

WU-505-90-5K

8. PERForMING ORGANIZATION REPORT NUMBER

E-9165

10. SPONSORINGMONITORING AGENCY REPORT NUMBER

NASA TM-106749

ICOMP-94-6

\section{SUPPLEMENTARY NOTES}

S.O. Ibraheem, Old Dominion University, Department of Mechanical Engineering, Norfolk, Virginia 23529; and A.O. Demuren, Institute for Computational Mechanics in Propulsion (work funded under NASA Cooperative Agreement NCC3-233), and Old Dominion University, Department of Mechanical Engineering, Norfolk, Virginia 23529. ICOMP Program Director, Louis A. Povinelli, organization code 2600, (216) 433-5818.

12a. DISTRIBUTIONAVAILABILITY STATEMENT

12b. DISTRIBUTION CODE

Unclassified - Unlimited

Subject Category 34

13. ABSTRACT (Maximum 200 words)

A procedure is presented for utilizing a bi-grid stability analysis as a practical tool for predicting multigrid performance in a range of numerical methods for solving Euler and Navier-Stokes equations. Model problems based on the convection, diffusion and Burger's equation are used to illustrate the superiority of the bi-grid analysis as a predictive tool for multigrid performance in comparison to the smoothing factor derived from conventional von Neumann analysis. For the Euler equations, bi-grid analysis is presented for three upwind difference based factorizations, namely Spatial, Eigenvalue and Combination splits, and two central difference based factorizations, namely LU and ADI methods. In the former, both the Steger-Warming and van Leer flux-vector splitting methods are considered. For the Navier-Stokes equations, only the Beam-Warming (ADI) central difference scheme is considered. In each case, estimates of multigrid convergence rates from the bi-grid analysis are compared to smoothing factors obtained from single-grid stability analysis. Effects of grid aspect ratio and flow skewness are examined. Both predictions are compared with practical multigrid convergence rates for 2-D Euler and Navier-Stokes solutions based on the Beam-Warming central scheme.

\section{SUBJECT TERMS}

Multigrid; Bi-grid local mode; Navier-Stokes

15. NUMBER OF PAGES

16. PRICE CODE

A04

\begin{tabular}{|c|c|}
\hline 17. SECURITY CLASSIFICATION & $\begin{array}{c}\text { 18. SECURITY CLASSIFICATION } \\
\text { OF REPORT } \\
\text { OF THIS PAGE } \\
\text { Unclassified }\end{array}$ \\
Unclassified
\end{tabular}

NSN 7540-01-280-5500
19. SECURTY CLASSIFICATION OF ABSTRACT Unclassified
1
20. LIMITATION OF ABSTRACT

Standard Form 298 (Rev. 2-89)

Prescribed by ANSI Std. Z39-18 298-102 
